\title{
Organocatalytic Domino Reaction of Spiroaziridine oxindoles and Malononitrile for the Enantiopure Synthesis of Spiro[dihydropyrrole-3,3'-oxindoles]
}

Saumen Hajra* ${ }^{* \dagger}$ SK Abu Saleh, ${ }^{\dagger \S}$ Atanu Hazra ${ }^{\dagger \S}$ and Maya Shankar Singh ${ }^{\S}$

$\dagger$ Centre of Biomedical Research, Sanjay Gandhi Post-Graduate Institute of Medical Sciences Campus, Raebareli Road, Lucknow 226014, INDIA

$\S$ Department of Chemistry, Institute of Science, Banaras Hindu University, Varanasi 221005, INDIA

Table of Contents

\begin{tabular}{|l|l|l|}
\hline 1 & Organocatalytic asymmetric domino reaction and kinetic resolution & S2-S3 \\
\hline 2 & ${ }^{1} \mathrm{H}$ and ${ }^{13} \mathrm{C}$ NMR spectra of compounds & S3-S28 \\
\hline 3 & X-ray crystal structure details & S28-S29 \\
\hline 4 & HPLC chromatogram of compounds & S30-S50 \\
\hline
\end{tabular}


Organocatalytic asymmetric domino reaction of spiroaziridine $( \pm)-1 a$ and its kinetic resolution:

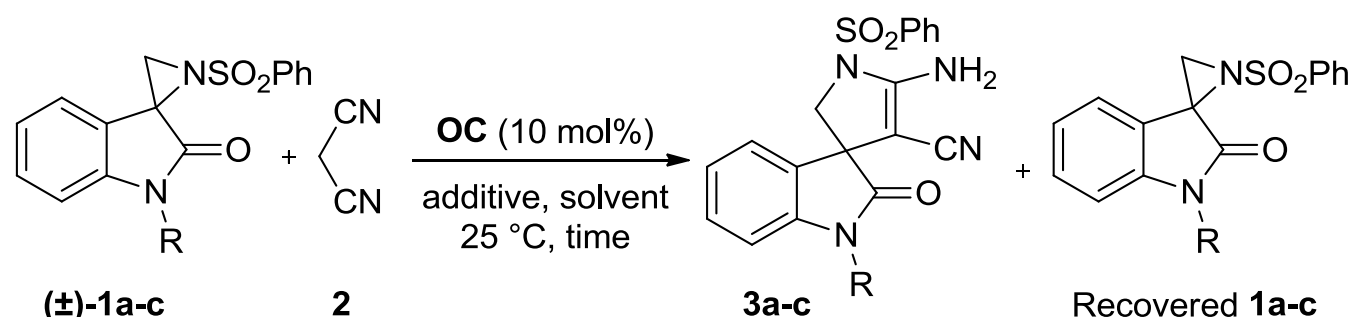<smiles>C=CC1=CCC([C@H](O)c2ccnc3ccc(OC)cc23)N=C1</smiles>

OC-1<smiles>C=CC1=CN2CCC([C@H](NC(=O)Nc3cc(C(F)(F)F)cc(C(F)(F)F)c3)c3ccnc4ccc(OC)cc34)CC2C1</smiles>

$\mathrm{OC}-2$<smiles>CN(C)C1CCCCC1NC(=S)Nc1cc(C(F)(F)F)cc(C(F)(F)F)c1</smiles>

OC-3<smiles>C=CC1=CN(Cc2ccccc2)[C@@H]([C@H](O)c2ccnc3ccc(OC)cc23)CC1</smiles>

OC-4<smiles>[R]OC(c1ccnc2ccccc12)[C@@H]1CCC(C=C)=CN1Cc1ccccc1</smiles>

OC-5 R = H

OC-6 R = benzoyl OC-7 R = adamantoyl

\begin{tabular}{|c|c|c|c|c|c|c|c|}
\hline entry & $\begin{array}{l}\text { Aziridine } \\
( \pm)-1\end{array}$ & catalyst & additive & solvent & time (h) & 3 (yield; ee) & $\begin{array}{l}\text { Recovered } 1 \text { (yield; } \\
\text { ee) }\end{array}$ \\
\hline 1 & $( \pm)-1 a$ & OC-1 & - & DMF & 9 & $29 \%$ 3a, 3\% ee & $40 \%$ 1a, racemic \\
\hline 2 & $( \pm)-1 a$ & OC-1 & - & THF & 9 & $31 \%$ 3a, $5 \%$ ee & $36 \%$ 1a, racemic \\
\hline 3 & $( \pm)-1 a$ & OC-1 & - & ethanol & 8 & $31 \%$ 3a, racemic & $35 \%$ 1a, racemic \\
\hline 4 & $( \pm)-1 a$ & OC-1 & - & $\mathrm{CHCl}_{3}$ & 10 & $28 \%$ 3a, racemic & $35 \%$ 1a, N.D \\
\hline 5 & $( \pm)-1 a$ & OC-1 & - & Toluene & 9 & $32 \% 3 a, 5 \%$ ee & $44 \%$ 1a, $18 \%$ ee \\
\hline 6 & $( \pm)-1 a$ & OC-1 & - & $\mathrm{CH}_{2} \mathrm{Cl}_{2}$ & 9 & $35 \%$ 3a, $12 \%$ ee & $45 \%$ 1a, $13 \%$ ee \\
\hline 7 & $( \pm)-1 a$ & OC-1 & - & $\mathrm{ClCH}_{2} \mathrm{CH}_{2} \mathrm{Cl}$ & 10 & $31 \%$ 3a, $6 \%$ ee & $39 \%$ 1a, N.D \\
\hline 8 & $( \pm)-1 a$ & OC-2 & - & $\mathrm{CH}_{2} \mathrm{Cl}_{2}$ & 10 & $30 \%$ 3a, $3 \%$ ee & $36 \%$ 1a, N.D \\
\hline 9 & $( \pm)-1 a$ & OC-3 & - & $\mathrm{CH}_{2} \mathrm{Cl}_{2}$ & 9 & $34 \%$ 3a, $6 \%$ ee & $40 \%$ 1a, racemic \\
\hline
\end{tabular}




\begin{tabular}{|c|c|c|c|c|c|c|c|}
\hline 10 & $( \pm)-1 a$ & OC-3 & - & Toluene & 10 & $31 \%$ 3a, $6 \%$ ee & $39 \%$ 1a, N.D \\
\hline 11 & $( \pm)-1 a$ & OC-4 & $\begin{array}{l}\mathrm{LiOH}(1 \\
\text { equiv) }\end{array}$ & Toluene & 8 & $31 \%$ 3a, racemic & $36 \%$ 1a, N.D \\
\hline 12 & $( \pm)-1 a$ & OC-5 & $\begin{array}{l}\mathrm{LiOH}(1 \\
\text { equiv) }\end{array}$ & Toluene & 9 & $28 \%$ 3a, racemic & $34 \%$ 1a, N.D \\
\hline 13 & $( \pm)-1 a$ & OC-6 & $\begin{array}{l}50 \% \text { aq. } \\
\mathrm{Cs}_{2} \mathrm{CO}_{3}\end{array}$ & Toluene & 8 & $30 \%$ 3a, racemic & $41 \%$ 1a, N.D \\
\hline 14 & $( \pm)-1 a$ & OC-7 & $\begin{array}{l}50 \% \text { aq. } \\
\mathrm{Cs}_{2} \mathrm{CO}_{3}\end{array}$ & Toluene & 10 & $30 \%$ 3a, racemic & $36 \%$ 1a, N.D \\
\hline 15 & $( \pm)-1 b$ & OC-1 & - & $\mathrm{CH}_{2} \mathrm{Cl}_{2}$ & 19 & $29 \%$ 3b, $9 \%$ ee & $35 \%$ 1b, N.D \\
\hline 16 & $( \pm)-1 c$ & OC-1 & - & $\mathrm{CH}_{2} \mathrm{Cl}_{2}$ & 22 & $25 \%$ 3c, racemic & $30 \%$ 1c, N.D \\
\hline
\end{tabular}
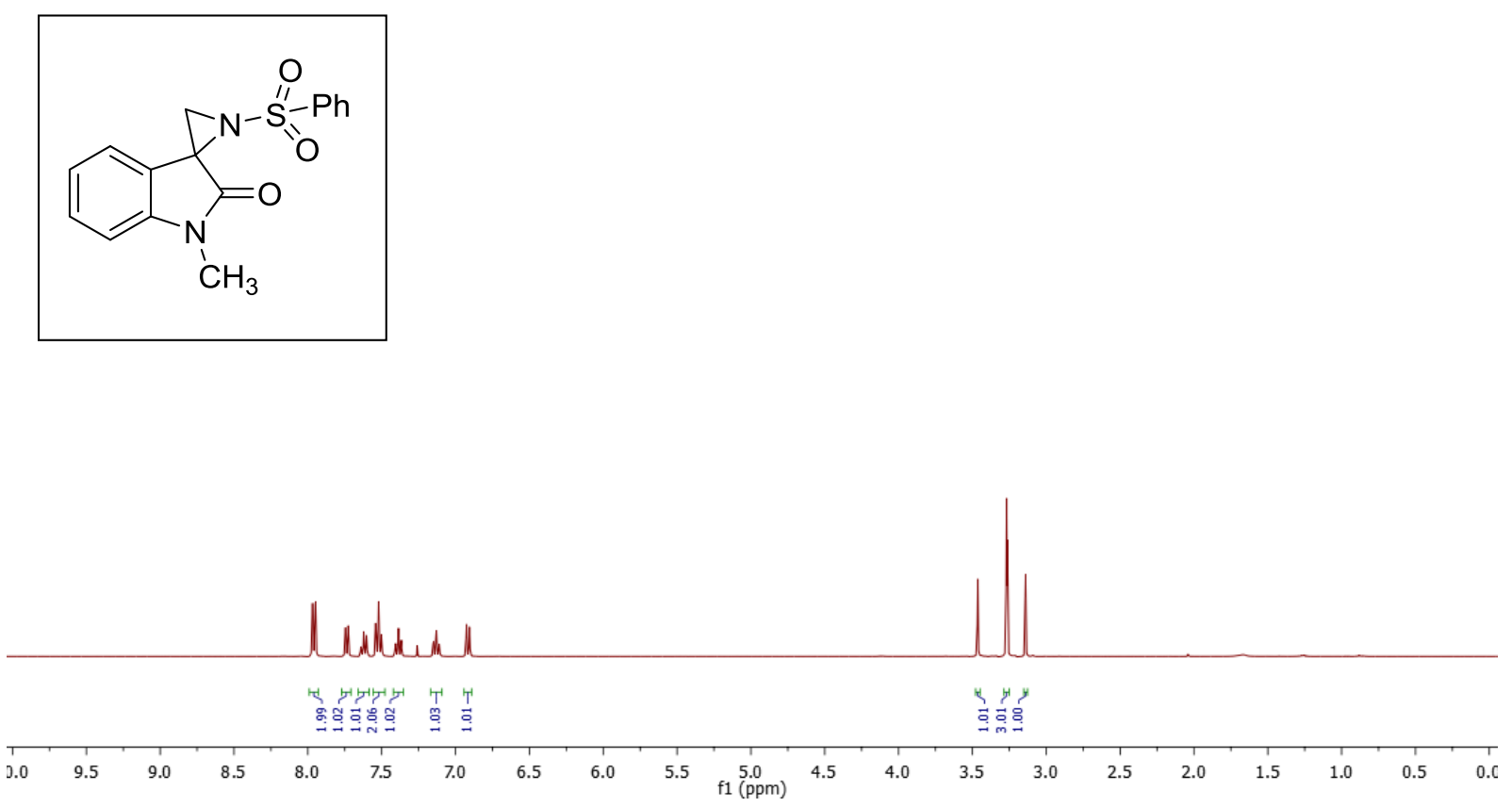

${ }^{1} \mathrm{H}$ NMR of compound ( \pm )-1a (400 $\left.\mathrm{MHz}, \mathrm{CDCl}_{3}\right)$ 

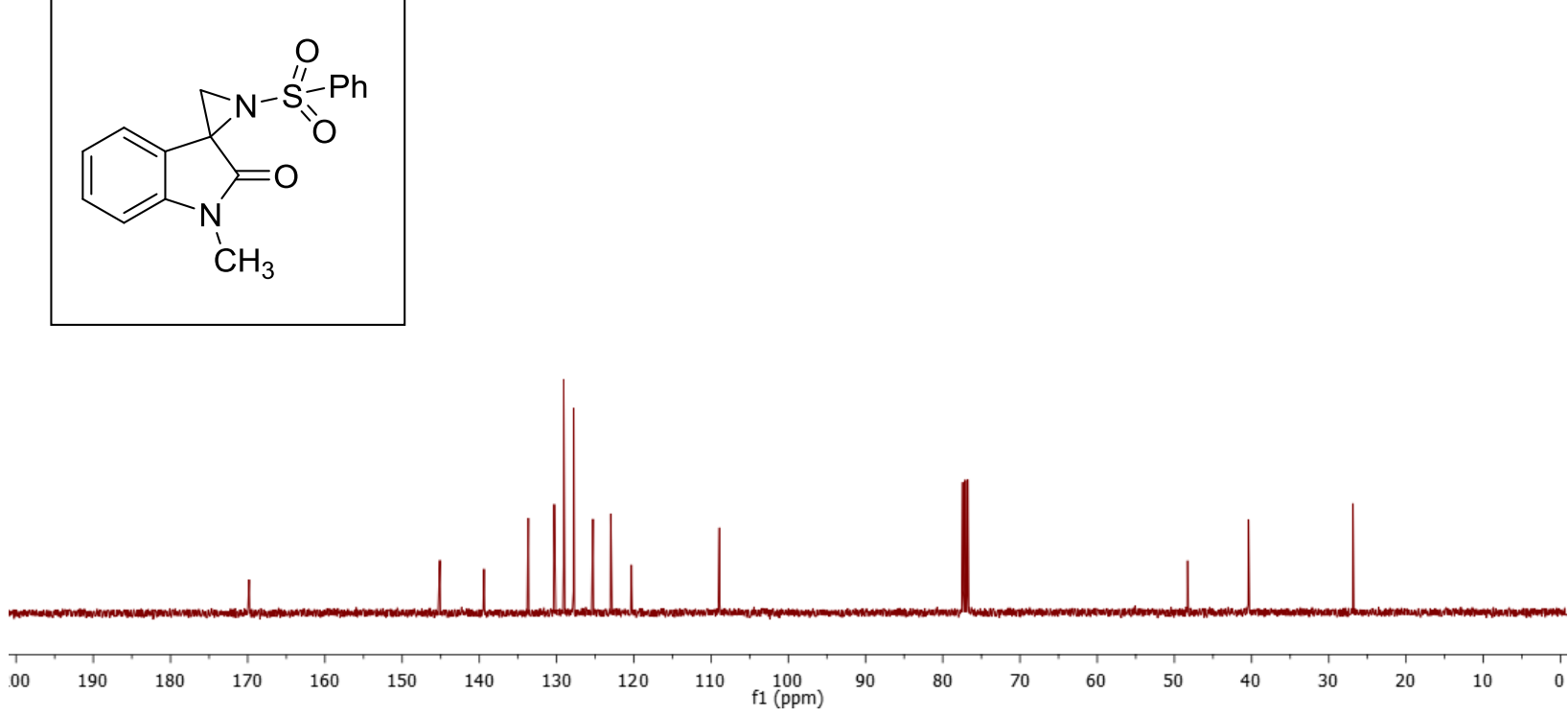

${ }^{13} \mathrm{C}$ NMR of compound ( \pm )-1a (100 $\left.\mathrm{MHz}, \mathrm{CDCl}_{3}\right)$

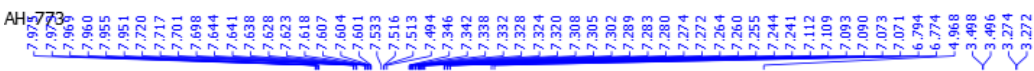
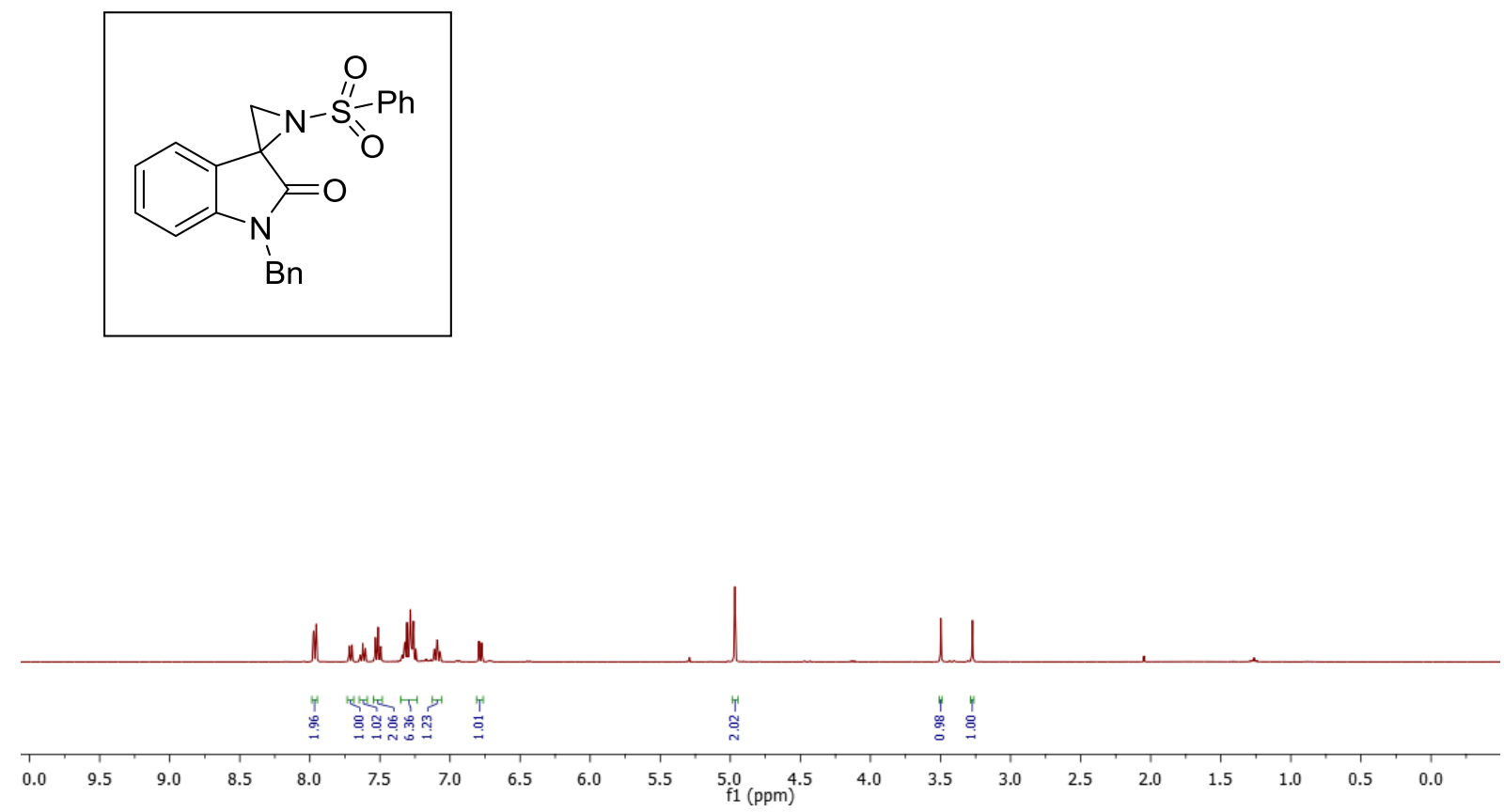

${ }^{1} \mathrm{H}$ NMR of compound $( \pm)-1 \mathrm{~b}\left(400 \mathrm{MHz}, \mathrm{CDCl}_{3}\right)$ 

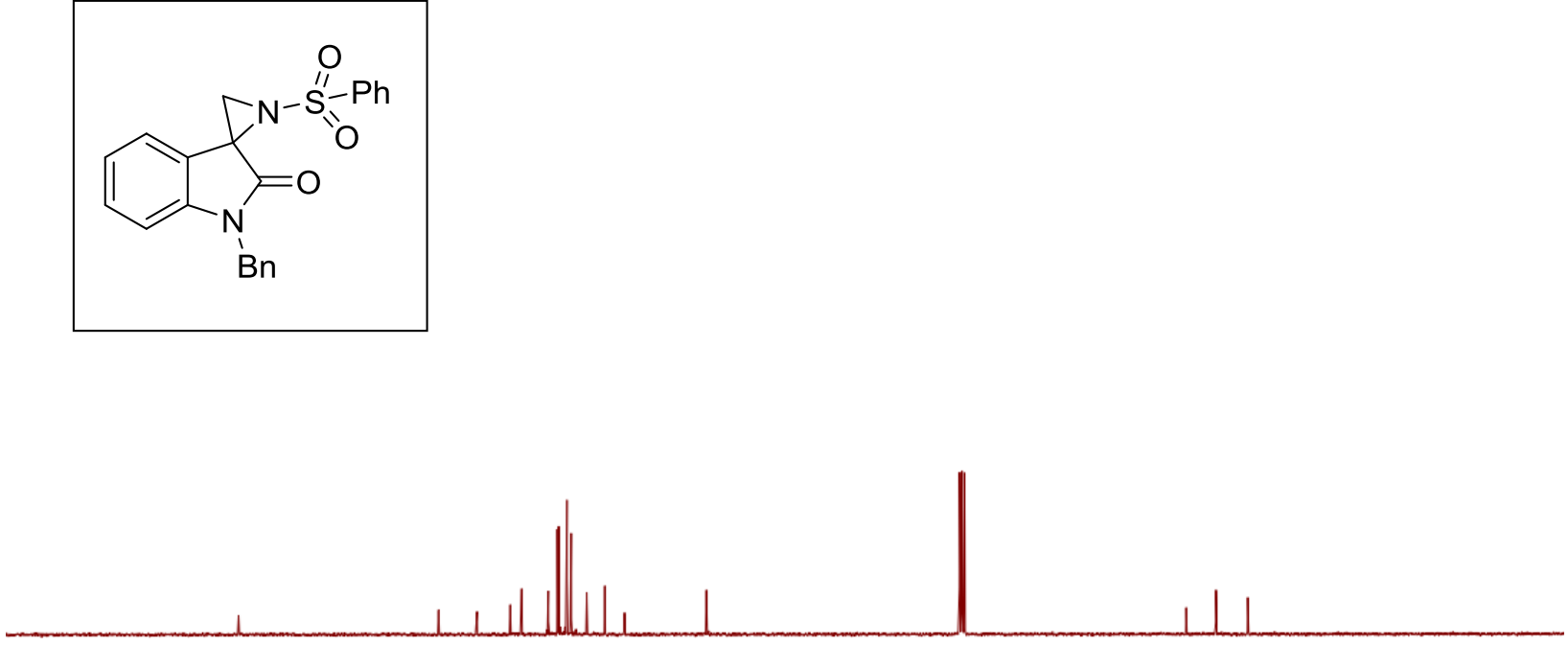

${ }^{13} \mathrm{C}$ NMR of compound ( \pm )-1b (100 $\left.\mathrm{MHz}, \mathrm{CDCl}_{3}\right)$

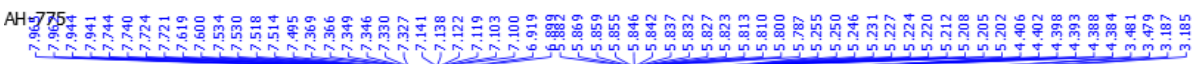
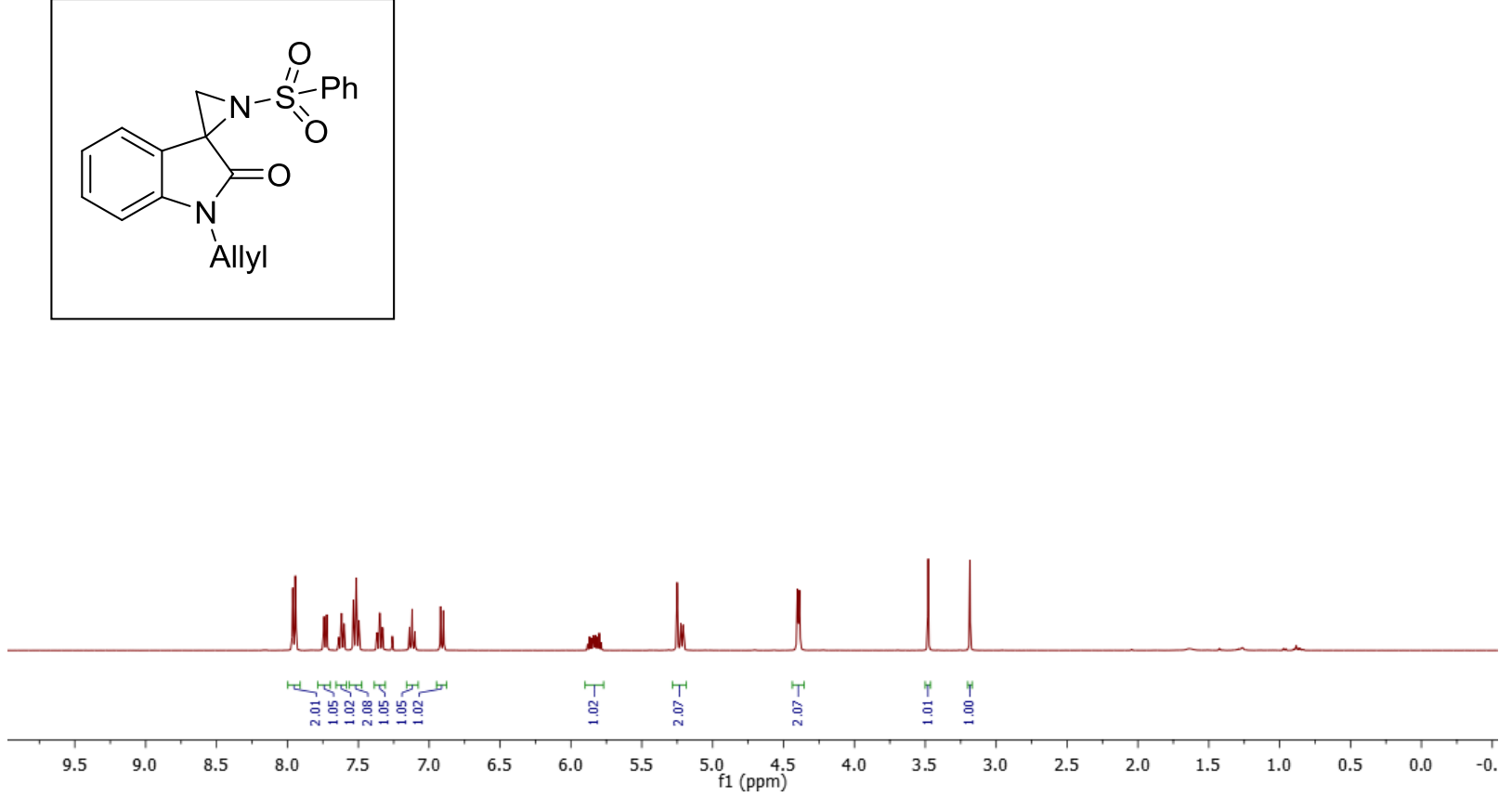

${ }^{1} \mathrm{H}$ NMR of compound ( \pm )-1c (400 $\left.\mathrm{MHz}, \mathrm{CDCl}_{3}\right)$ 

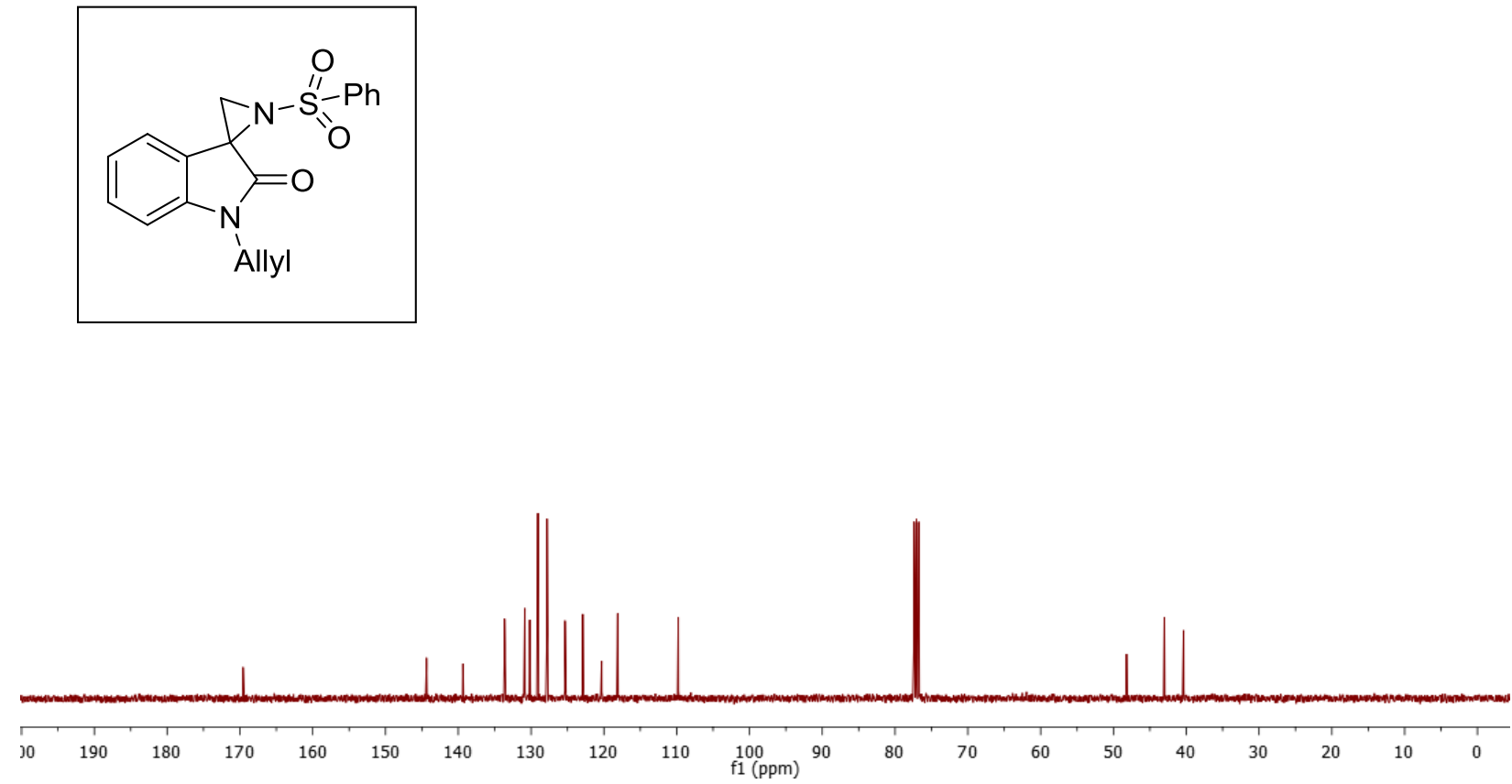

${ }^{13} \mathrm{C}$ NMR of compound ( \pm )-1c (100 $\left.\mathrm{MHz}, \mathrm{CDCl}_{3}\right)$
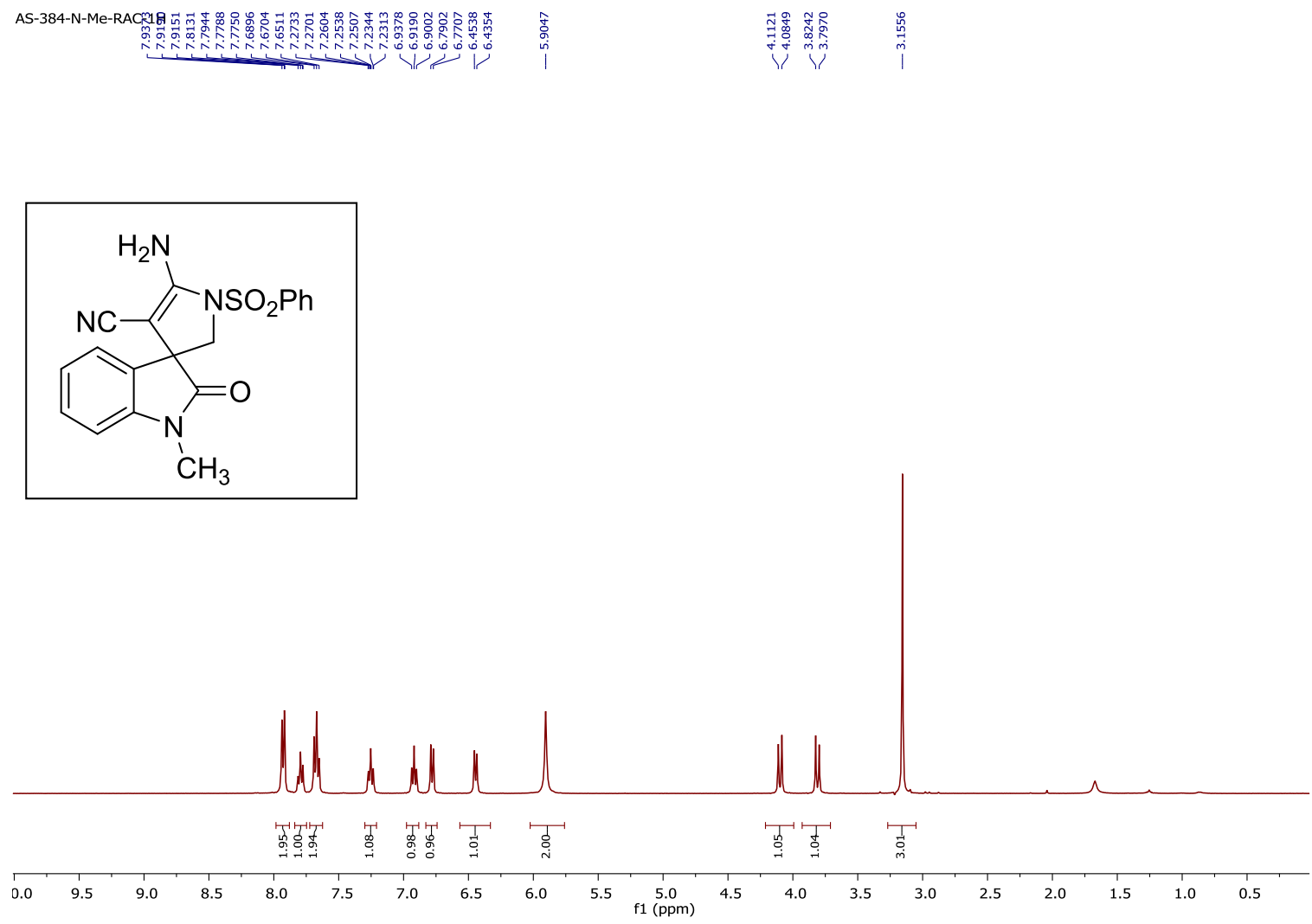

${ }^{1} \mathrm{H}$ NMR of compound ( \pm )-3a (400 $\left.\mathrm{MHz}, \mathrm{CDCl}_{3}\right)$ 

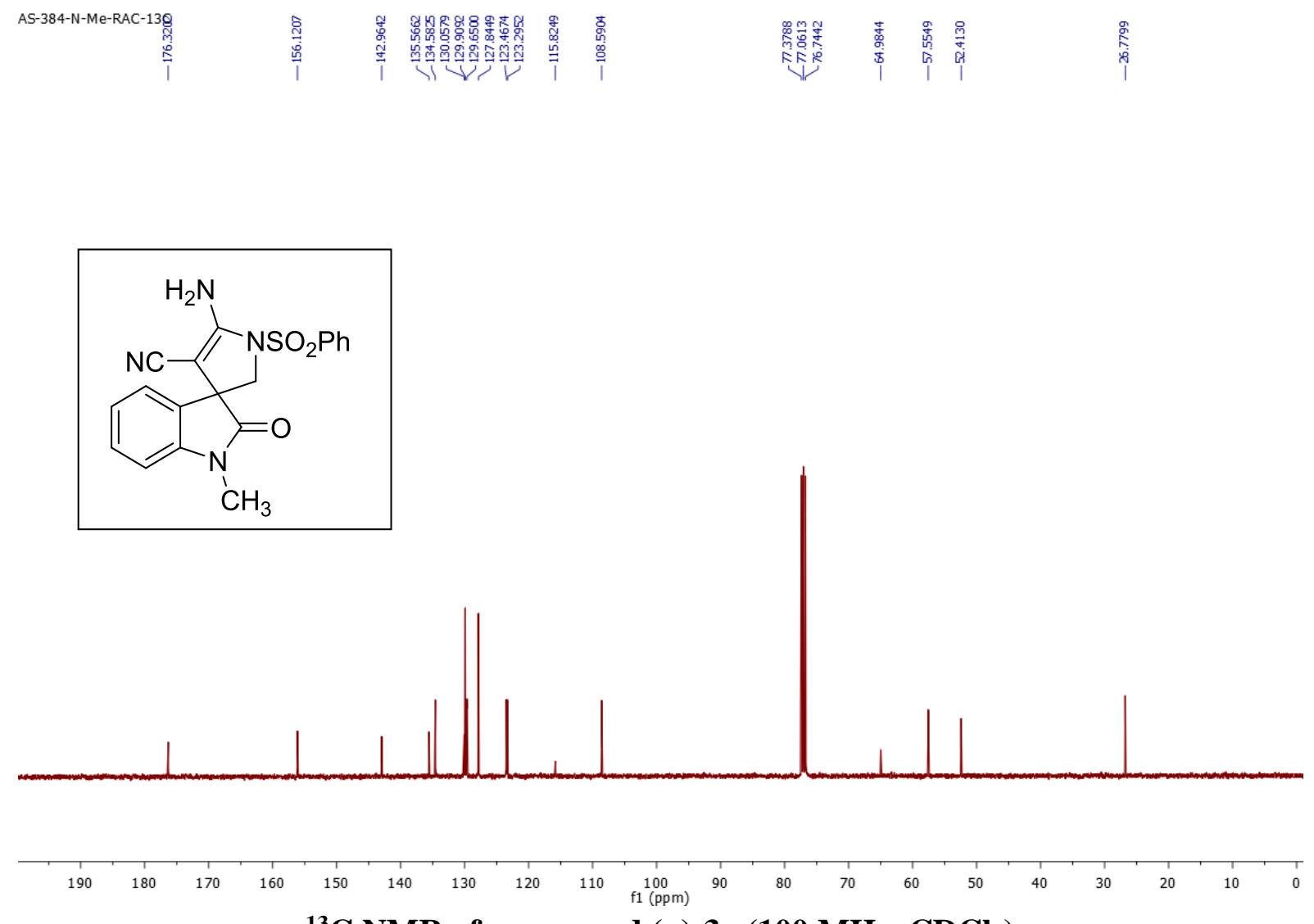

${ }^{13} \mathrm{C}$ NMR of compound ( \pm )-3a (100 $\left.\mathrm{MHz}, \mathrm{CDCl}_{3}\right)$
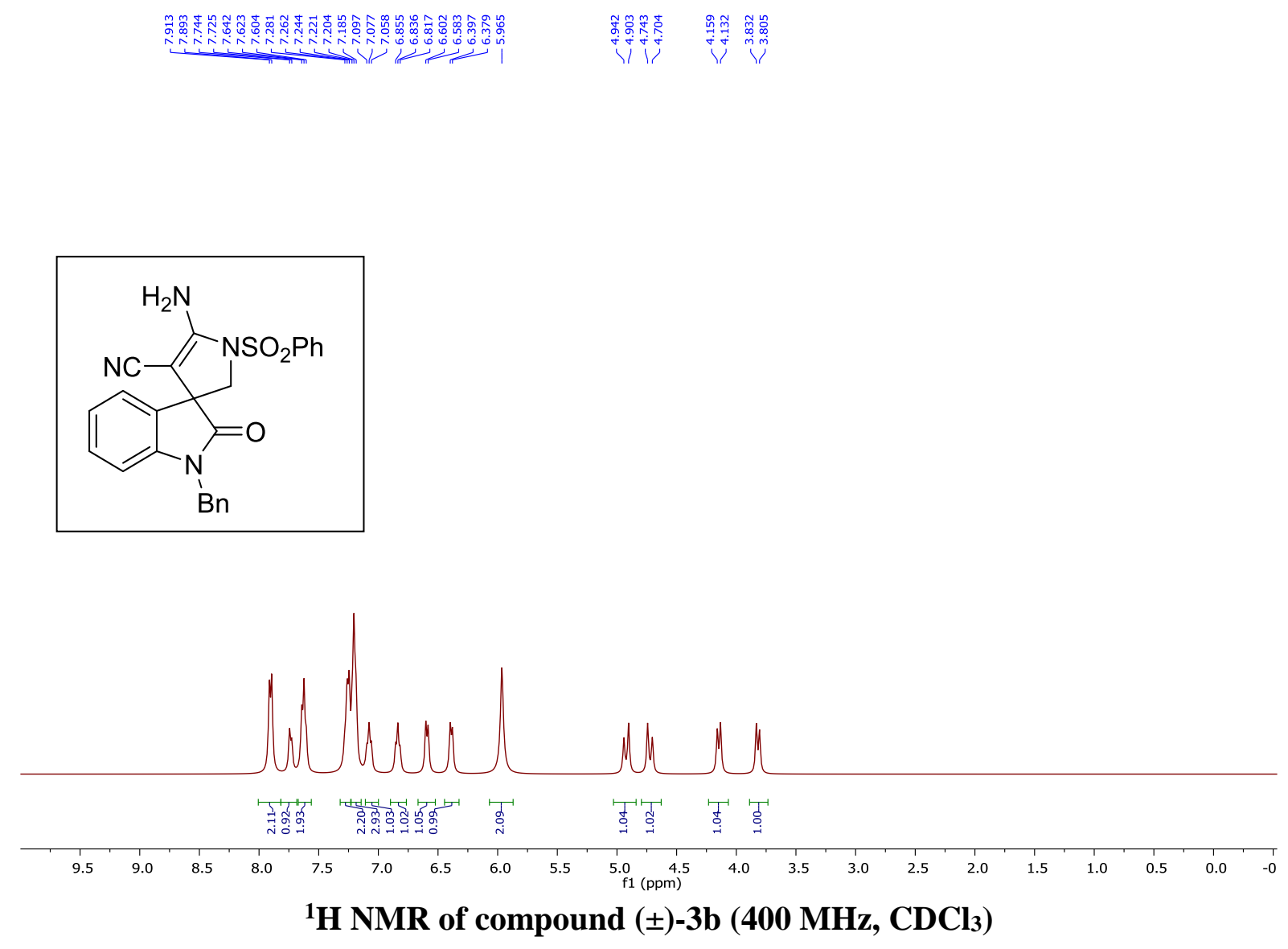

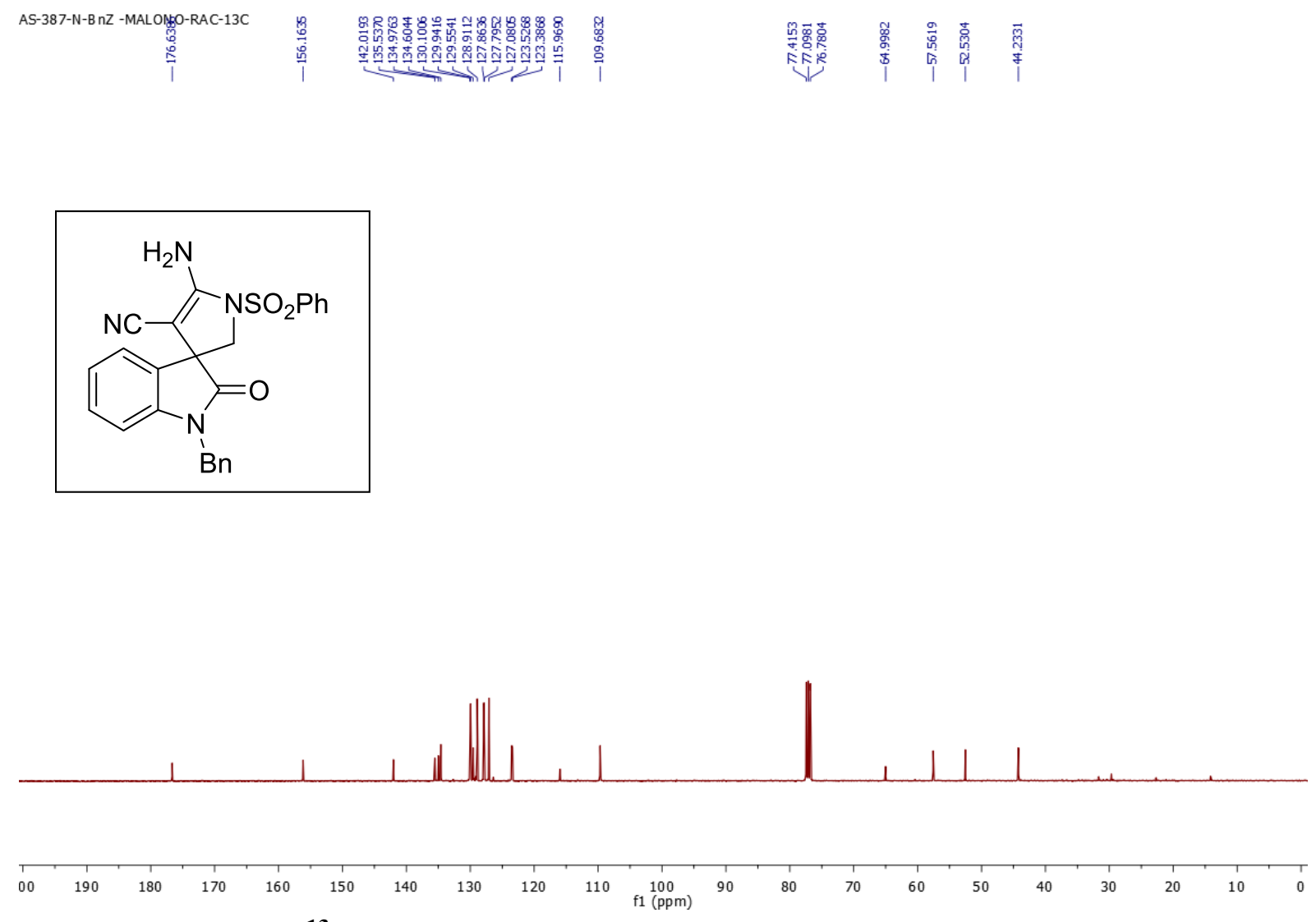

${ }^{13} \mathrm{C}$ NMR of compound $( \pm)-3 \mathrm{~b}\left(100 \mathrm{MHz}, \mathrm{CDCl}_{3}\right)$

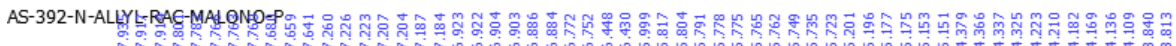

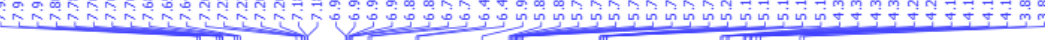
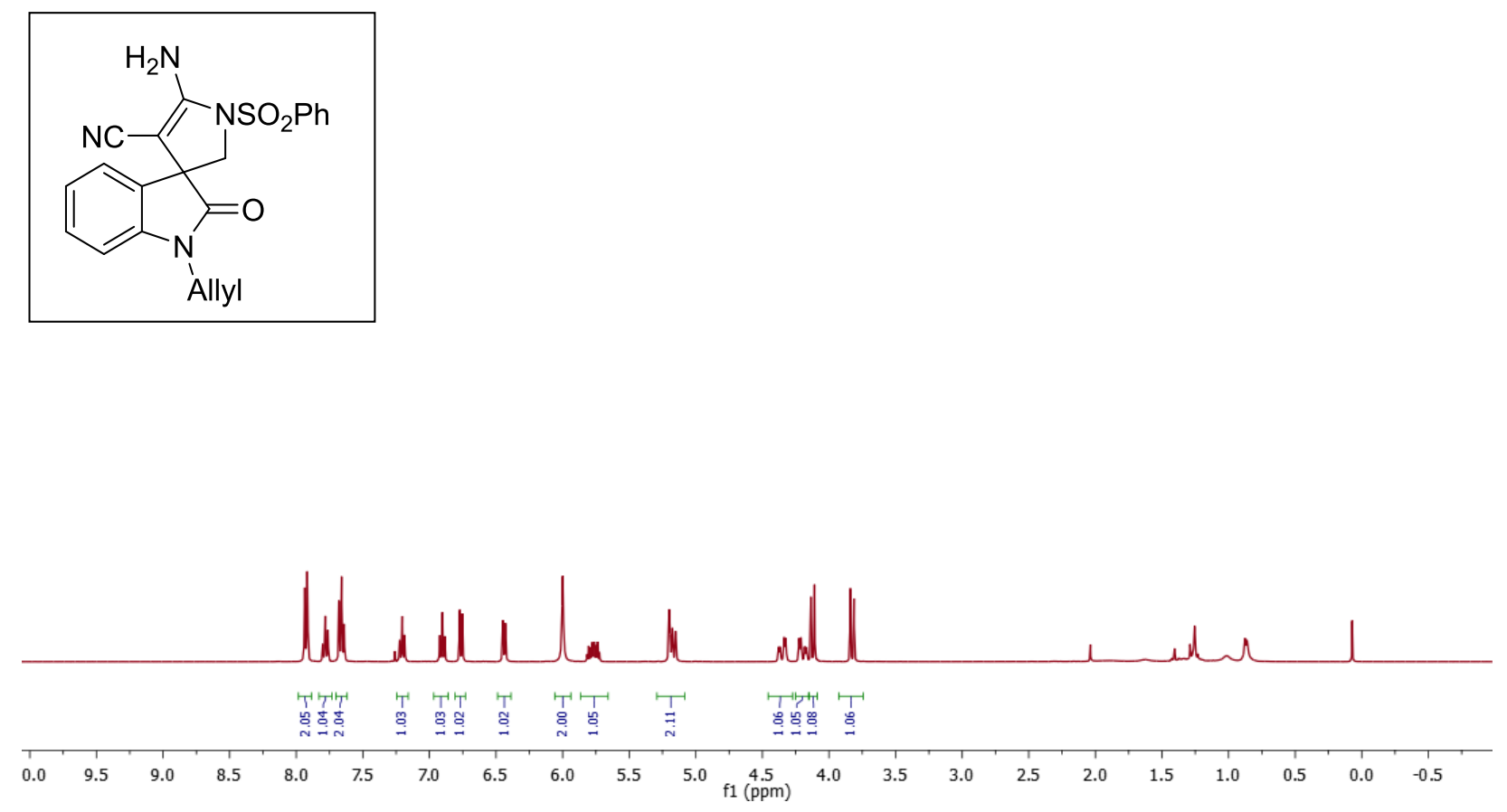

${ }^{1} \mathrm{H}$ NMR of compound ( \pm )-3c (400 $\left.\mathrm{MHz}, \mathrm{CDCl}_{3}\right)$ 

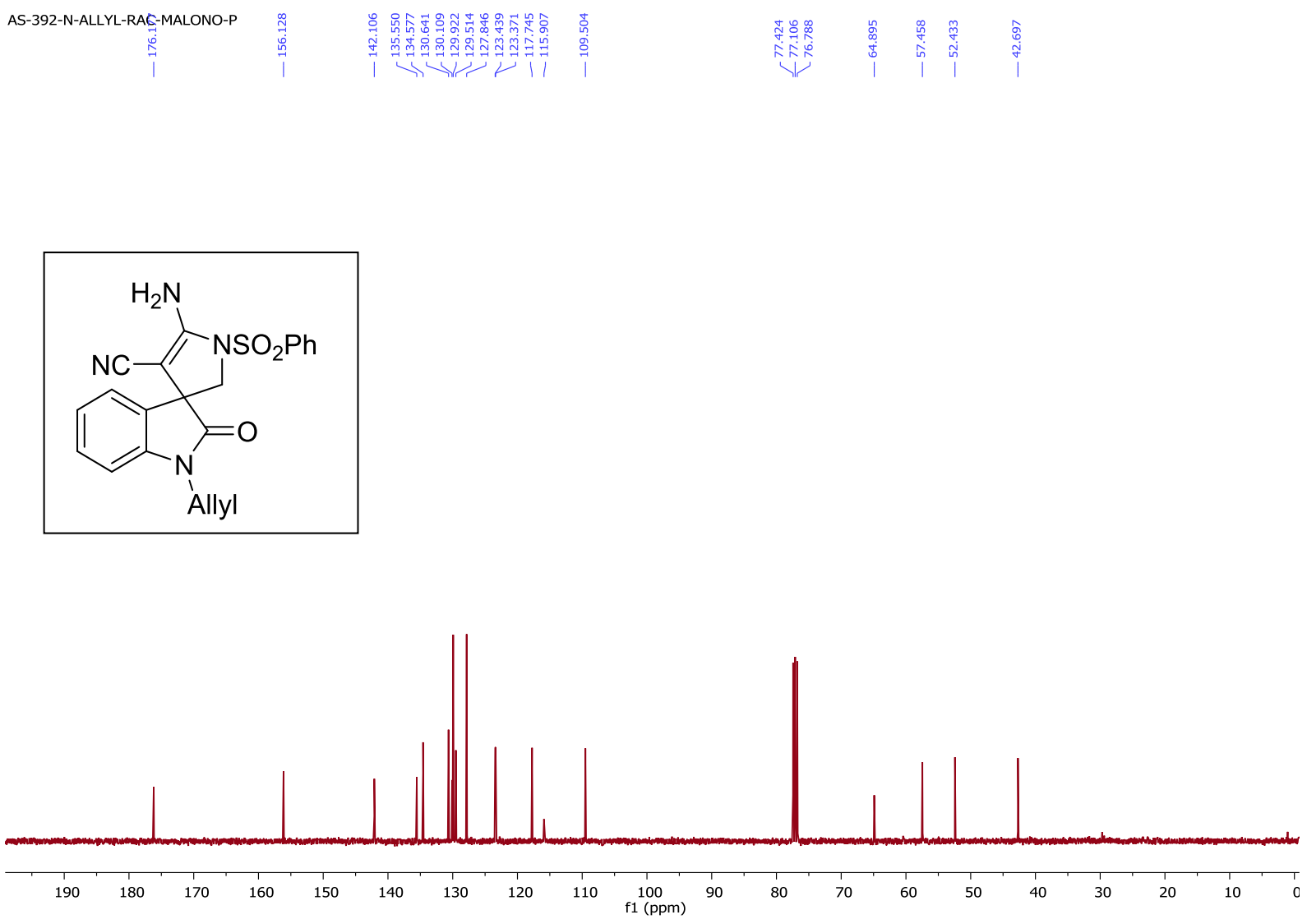

${ }^{13} \mathrm{C}$ NMR of compound $( \pm)-3 \mathrm{c}\left(100 \mathrm{MHz}, \mathrm{CDCl}_{3}\right)$

AS-298-R-P

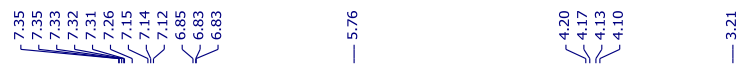

ำ
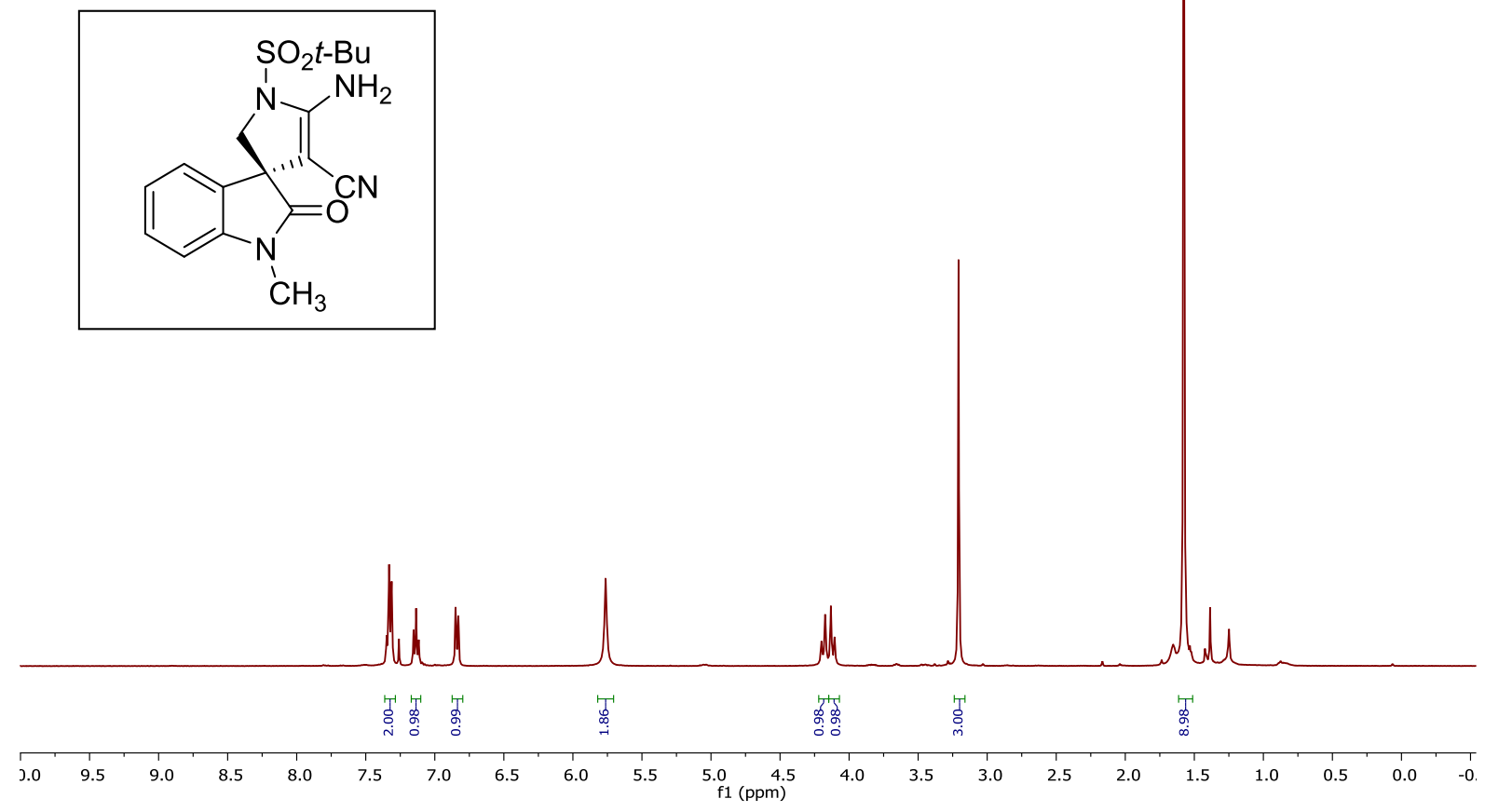

${ }^{1} \mathrm{H}$ NMR of compound 3d (400 $\left.\mathrm{MHz}, \mathrm{CDCl}_{3}\right)$ 

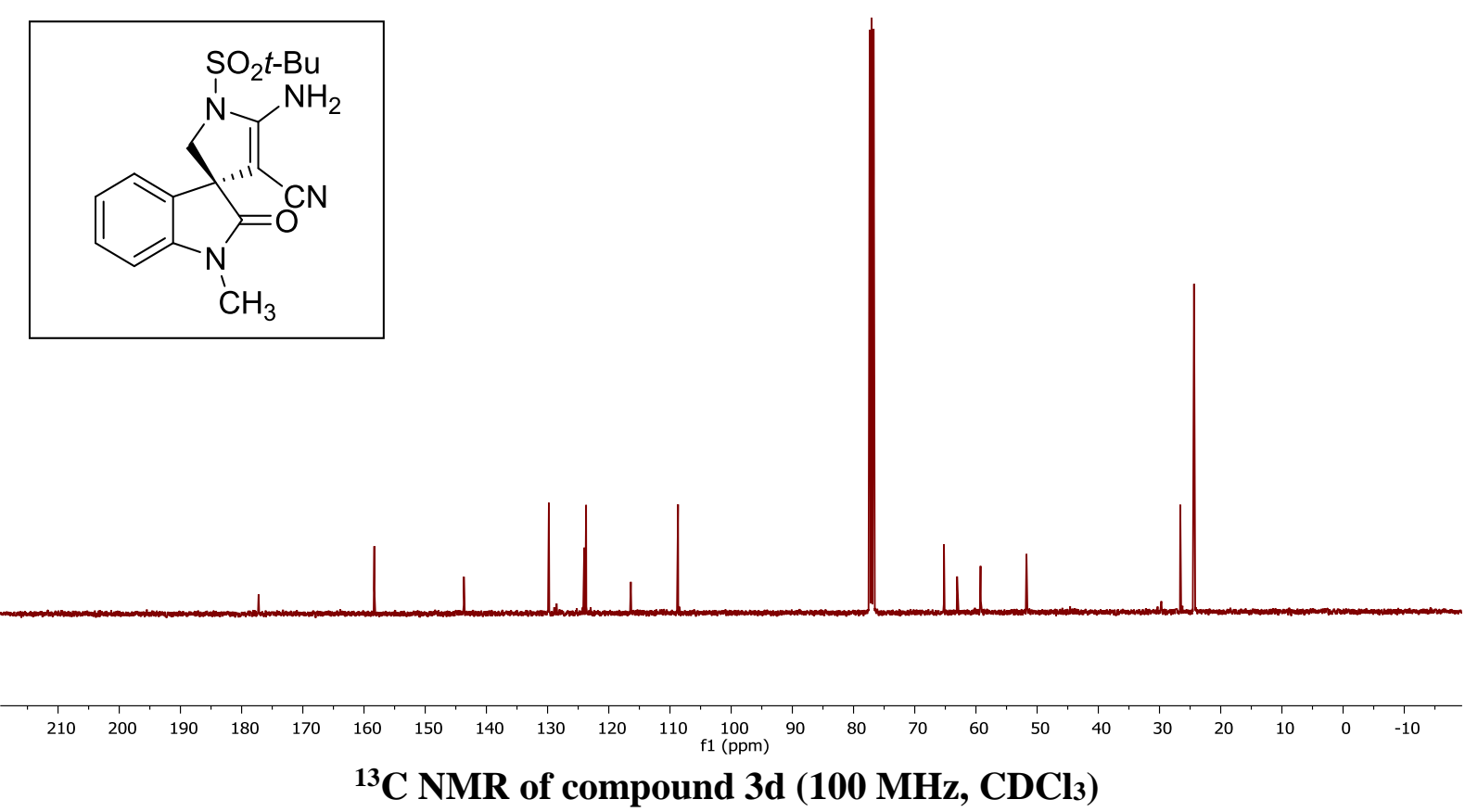

sh-as-306-p
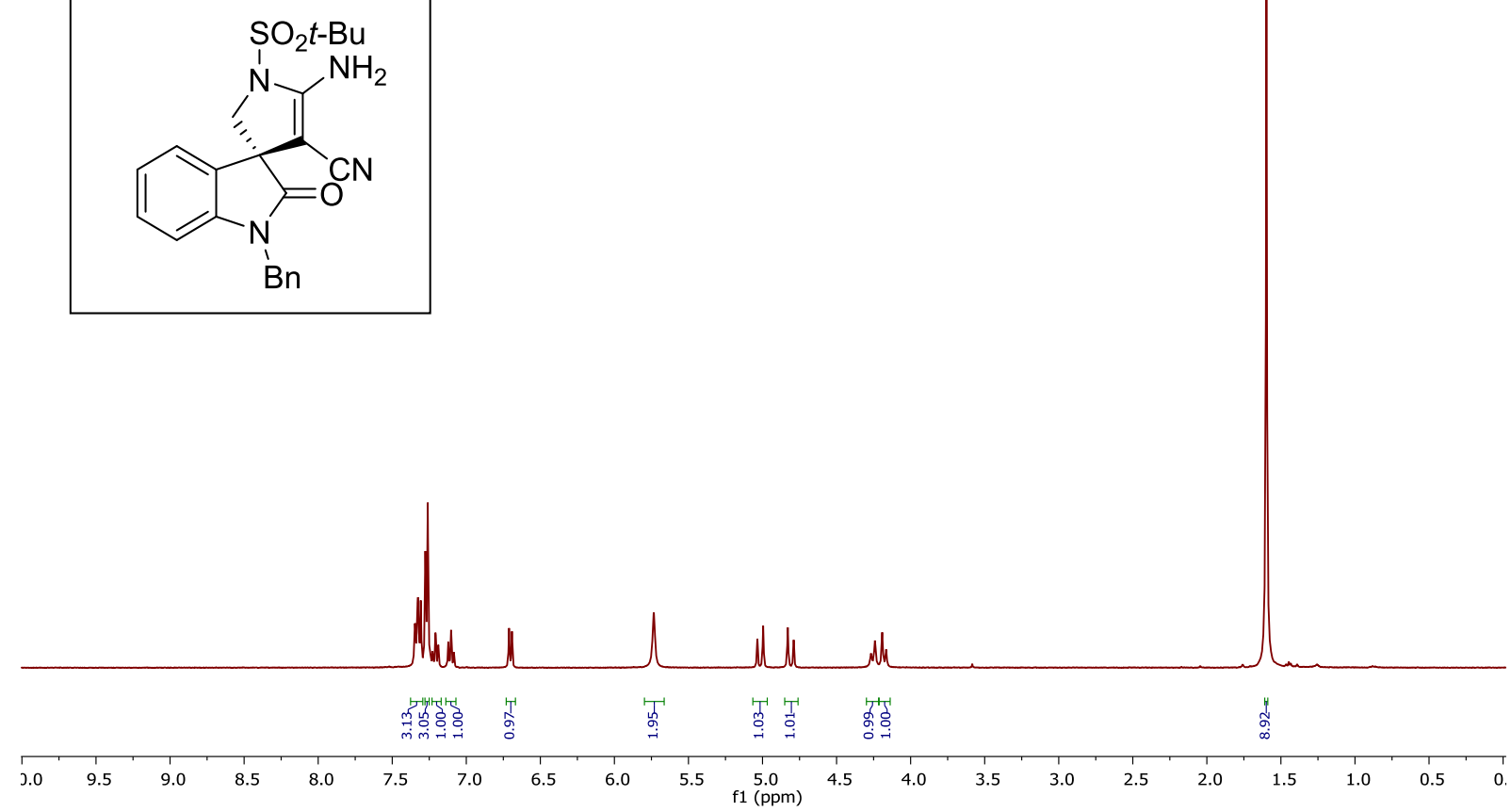

${ }^{1} \mathrm{H}$ NMR of compound ent-3e (400 $\mathrm{MHz}^{\left.-\mathrm{CDCl}_{3}\right)}$ 

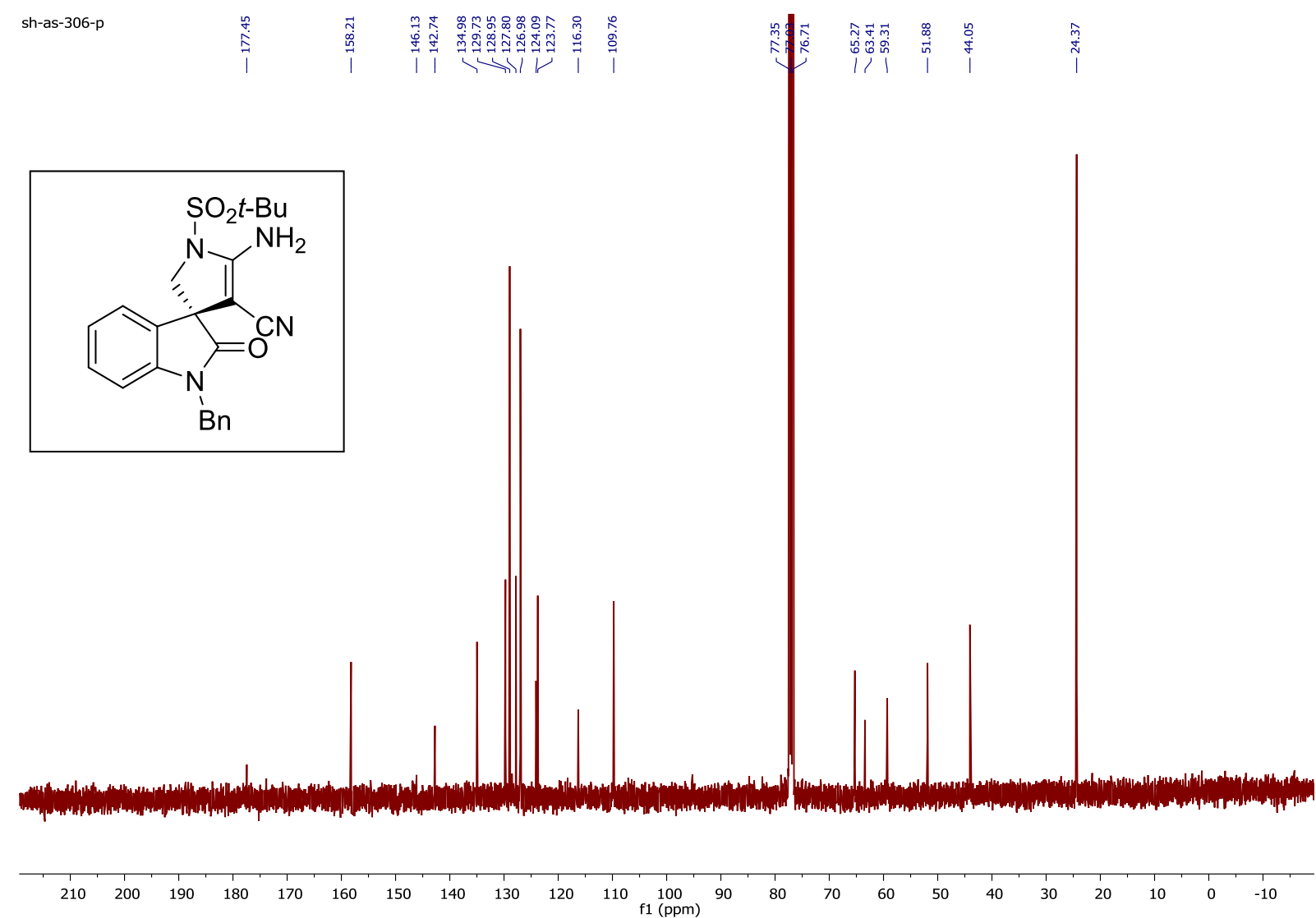

${ }^{13} \mathrm{C}$ NMR of compound ent-3e (100 $\left.\mathrm{MHz}, \mathrm{CDCl}_{3}\right)$
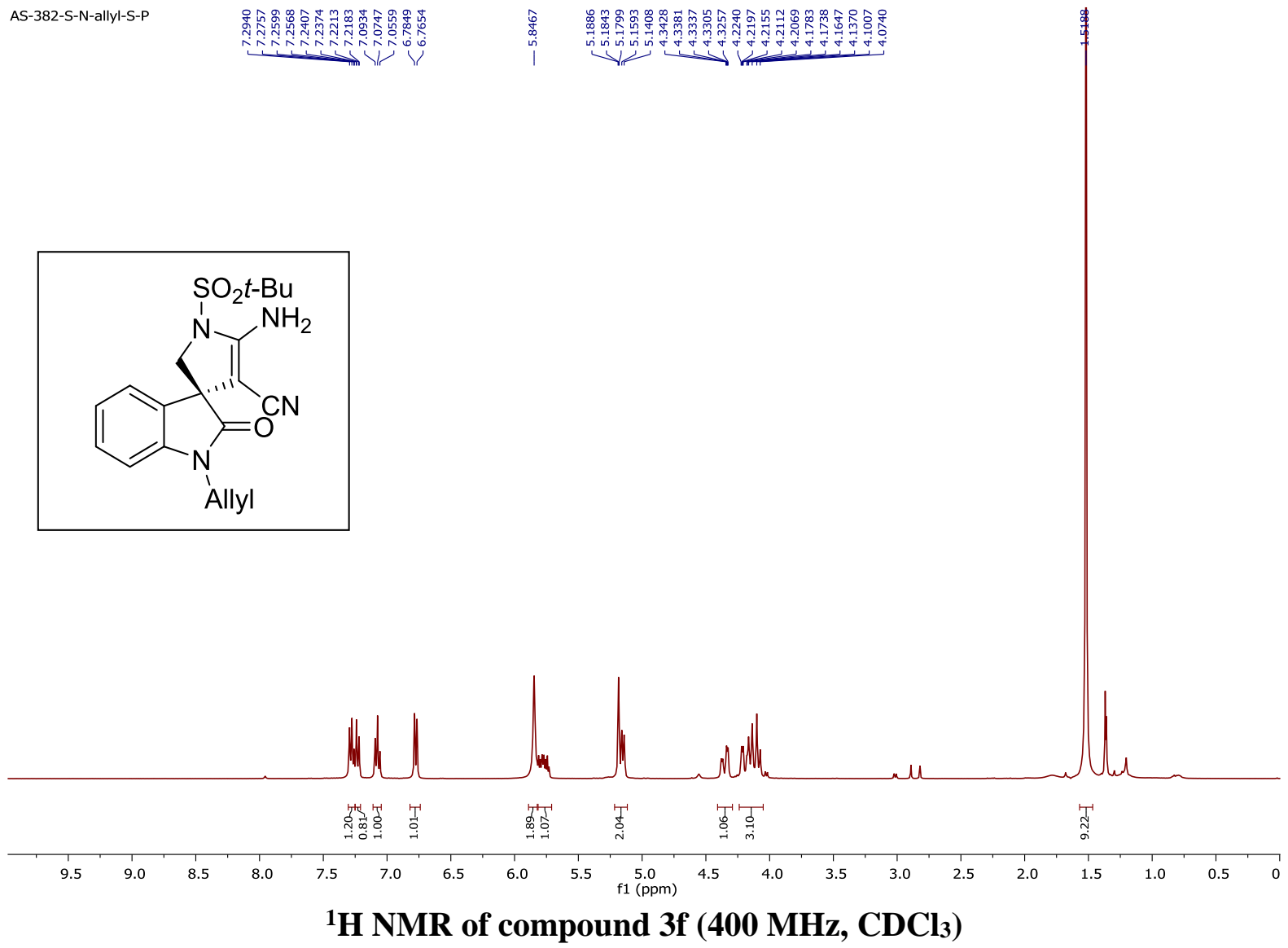

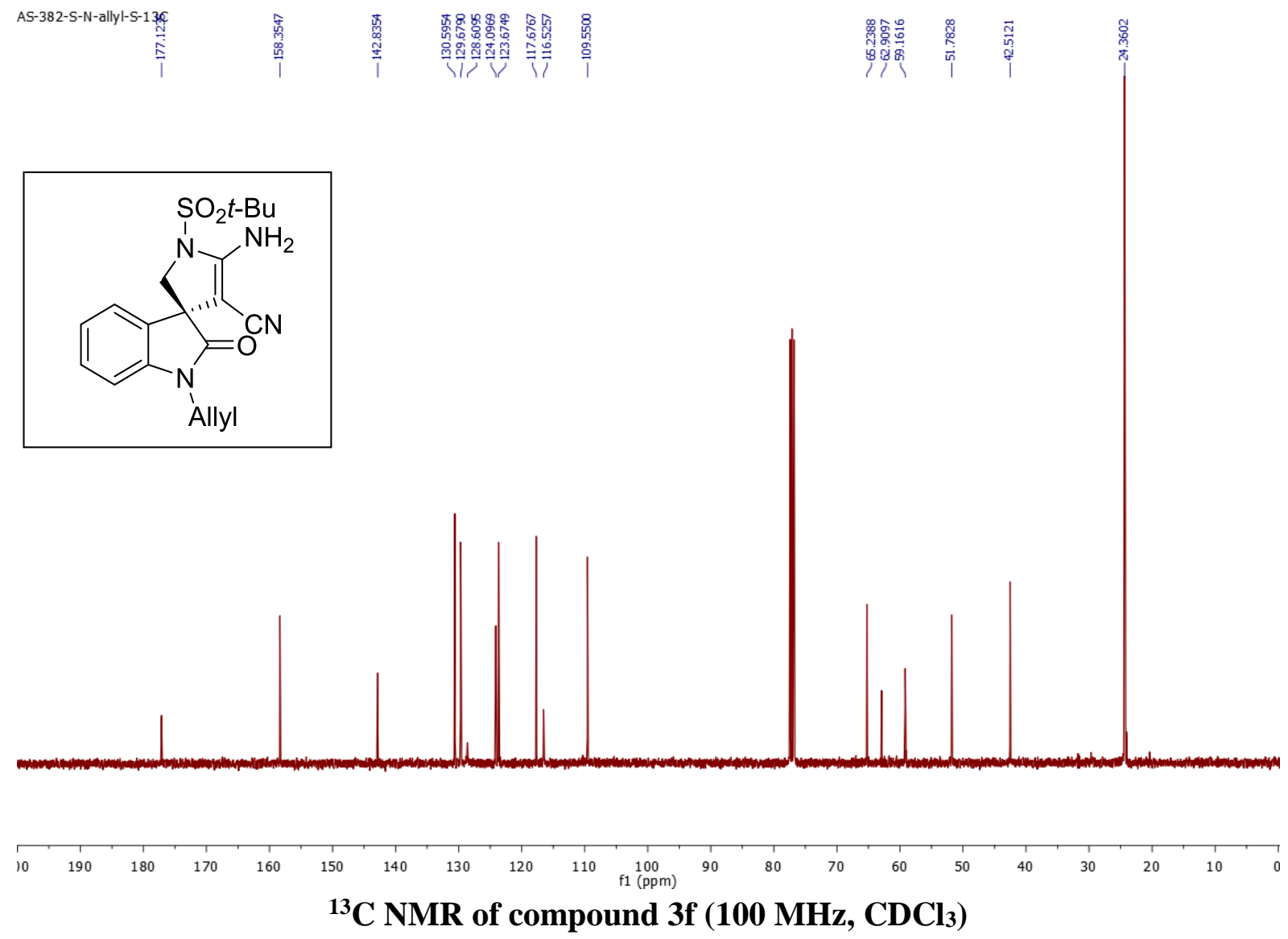

AS-N-BOC-R-MALONO-1H
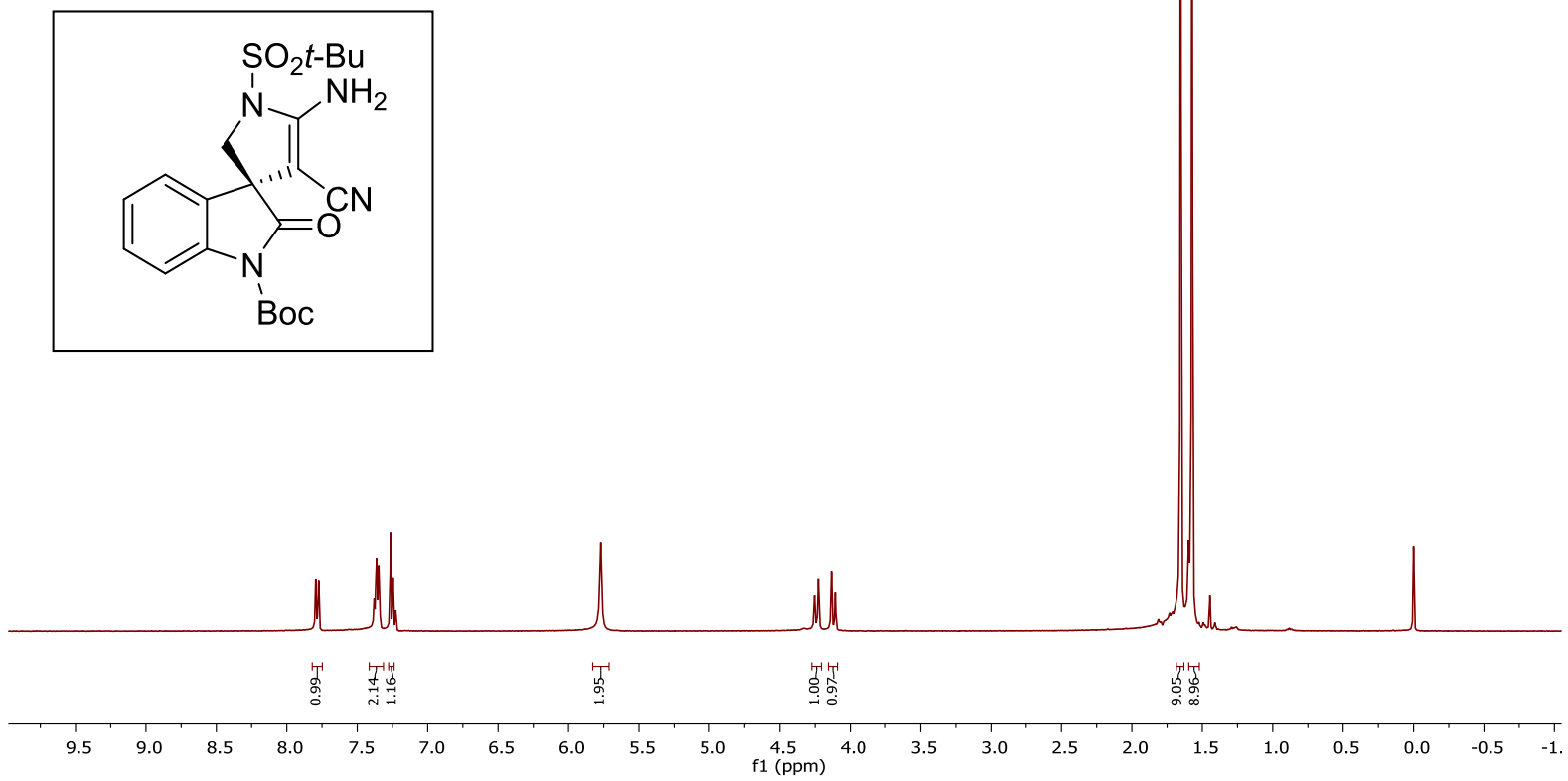

${ }^{\mathbf{1}} \mathrm{H}$ NMR of compound $\mathbf{3 g}\left(\mathbf{4 0 0} \mathrm{MHz}, \mathrm{CDCl}_{3}\right)$ 

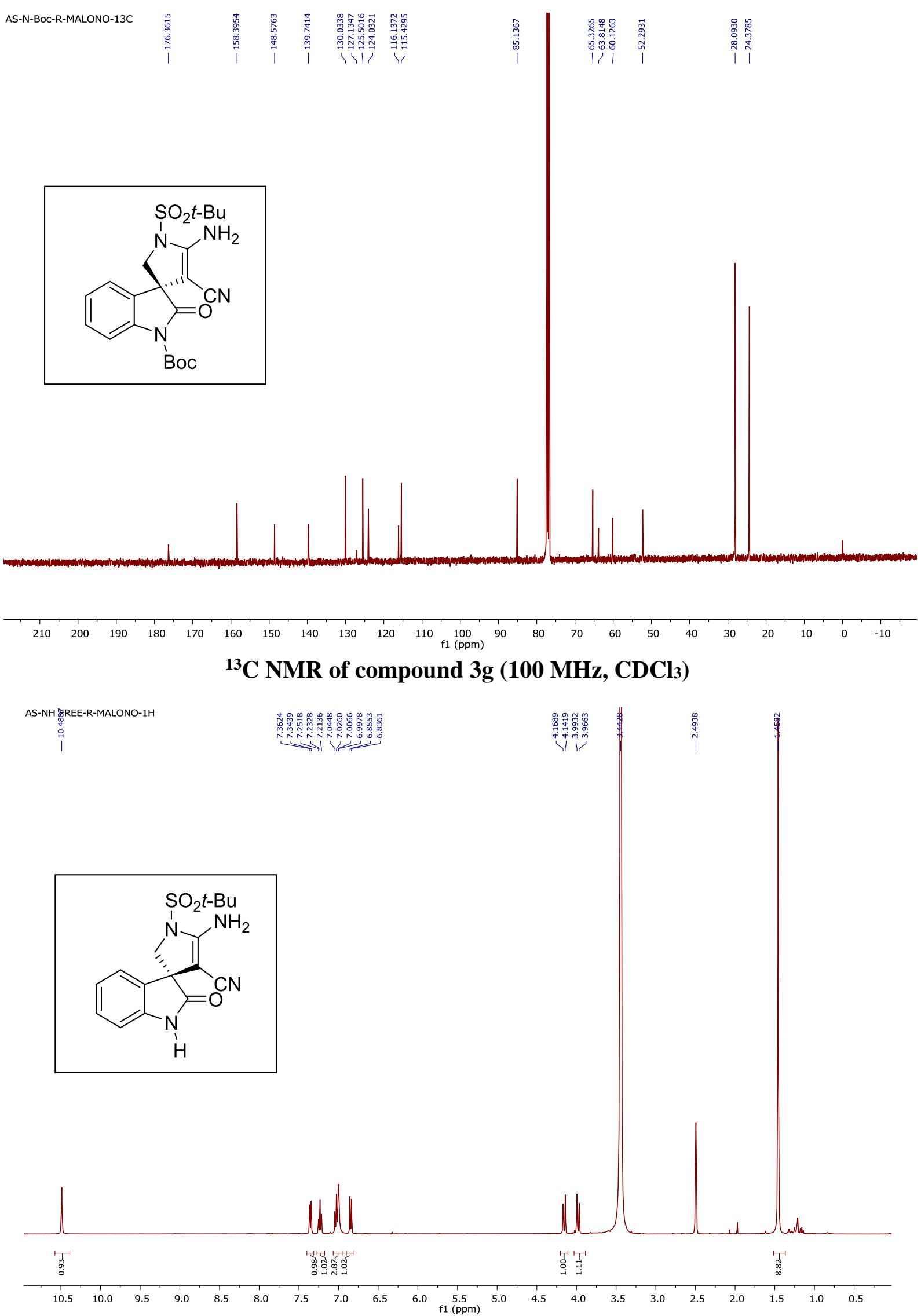

${ }^{1}$ H NMR of compound ent-3h (400 MHz, DMSO-d6) 


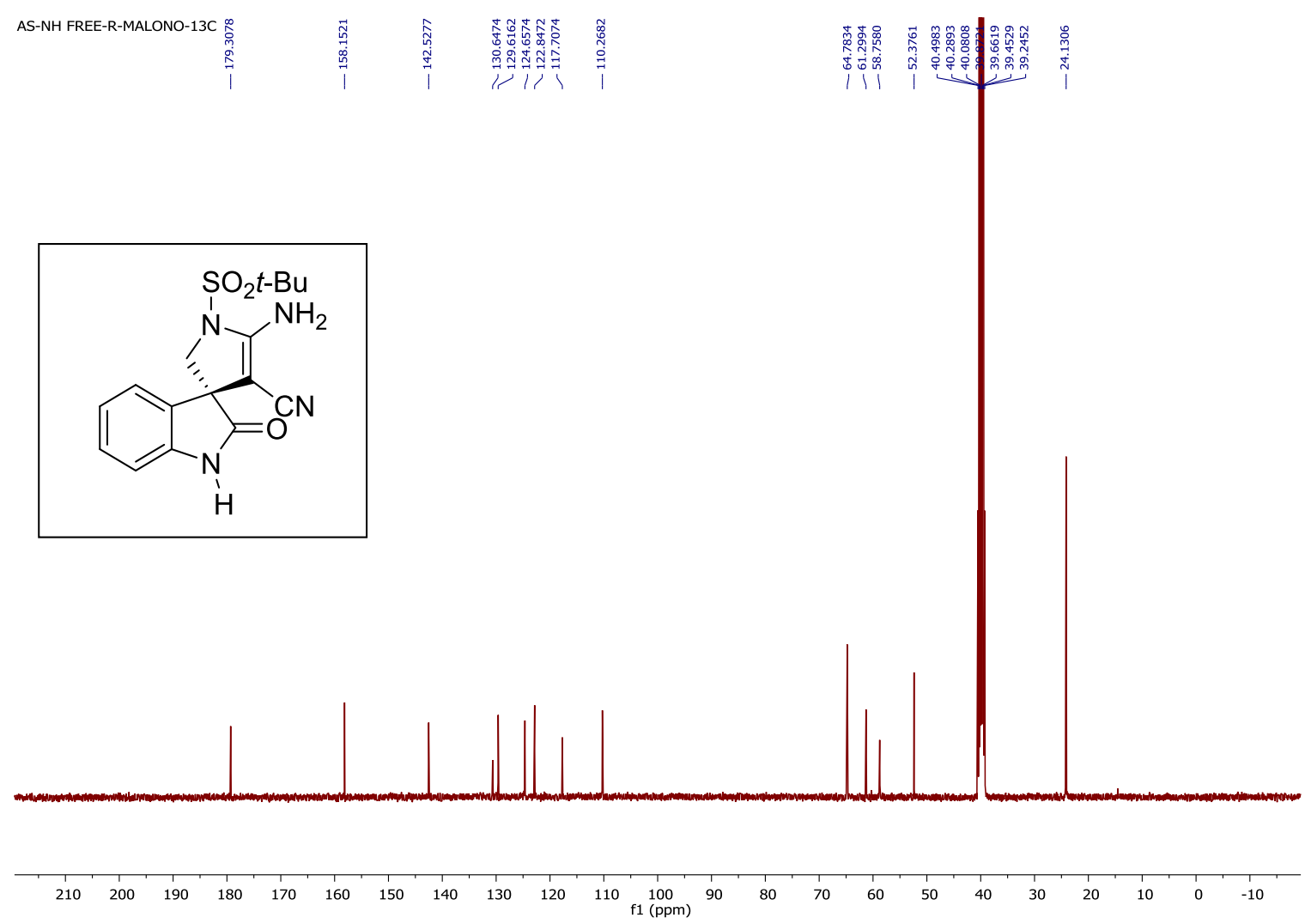

${ }^{13} \mathrm{C}$ NMR of compound ent-3h (100 MHz, DMSO-d6)

AS-338-5-OMe-R-P

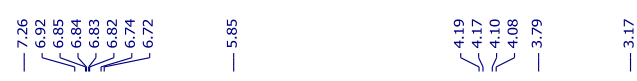
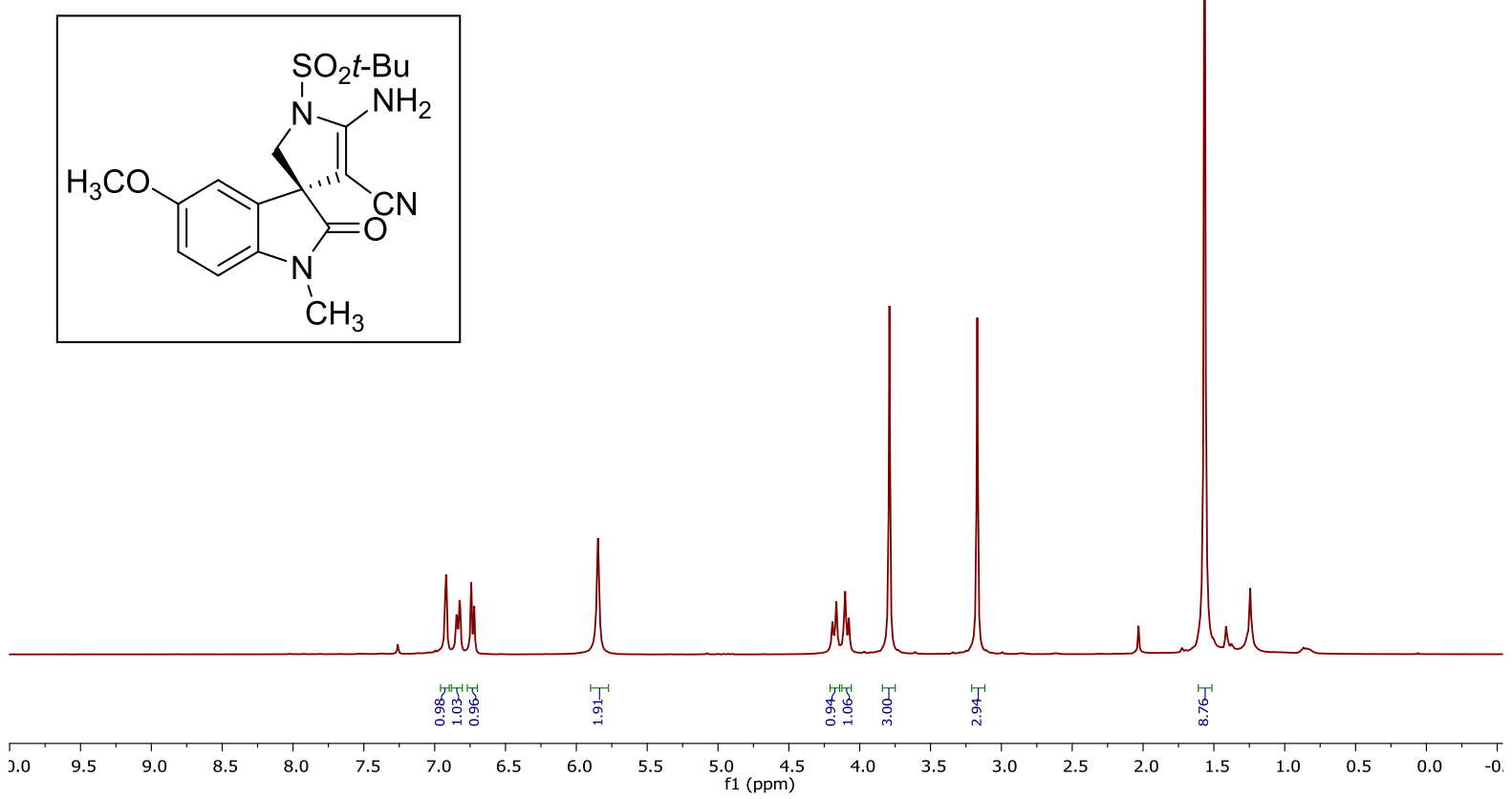

${ }^{1} \mathrm{H}$ NMR of compound $3 \mathrm{i}\left(400 \mathrm{MHz}, \mathrm{CDCl}_{3}\right)$ 

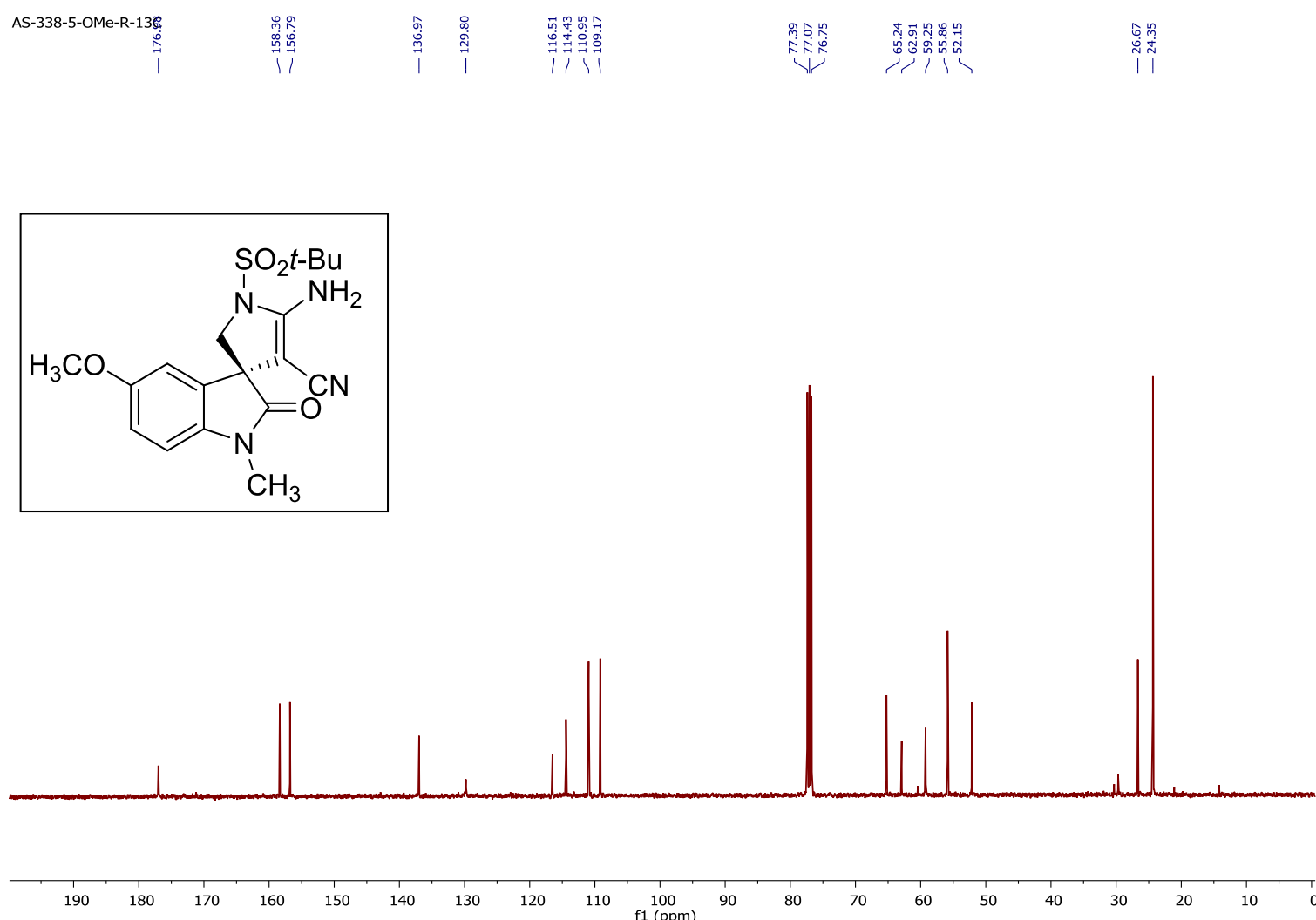

${ }^{13} \mathrm{C}$ NMR of compound 3i (100 $\left.\mathrm{MHz}, \mathrm{CDCl}_{3}\right)$

AS-375-R-P

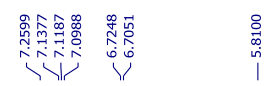

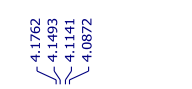
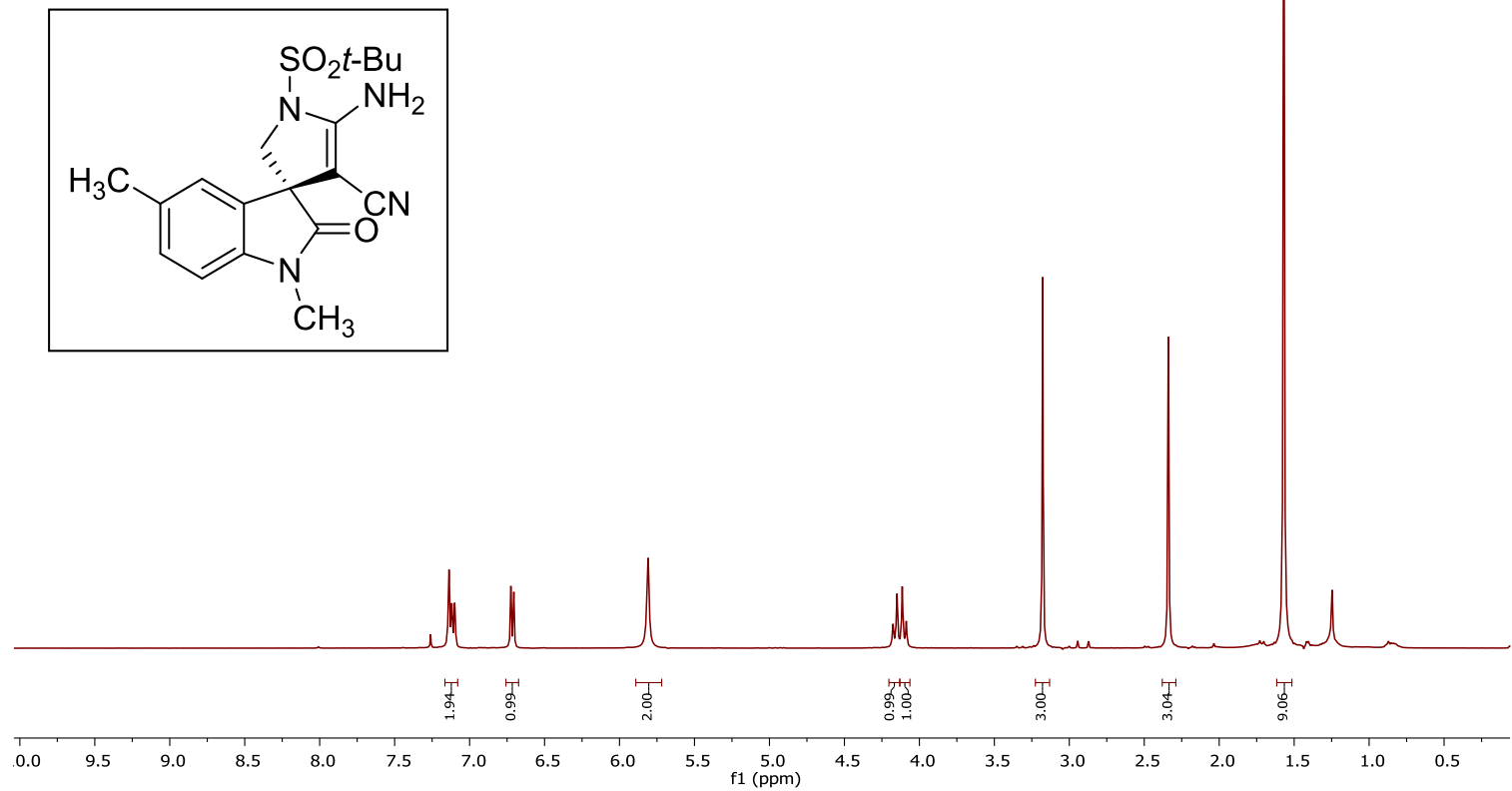

${ }^{1} \mathrm{H}$ NMR of compound ent-3j (400 $\left.\mathrm{MHz}, \mathrm{CDCl}_{3}\right)$ 

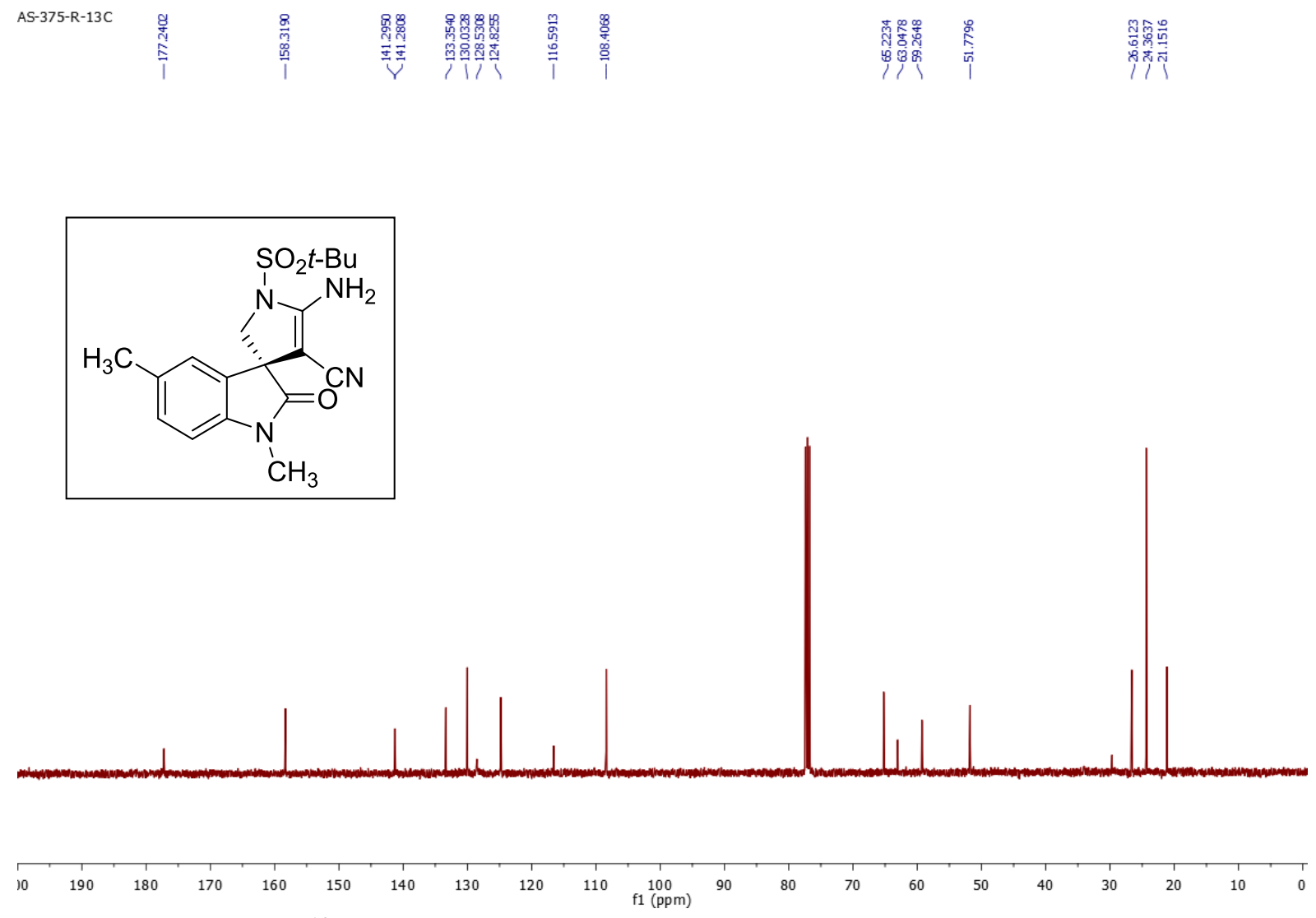

${ }^{13} \mathrm{C}$ NMR of compound ent-3j (100 $\left.\mathrm{MHz}, \mathrm{CDCl}_{3}\right)$

SH-AS-324-S-P

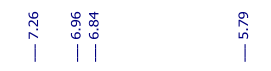

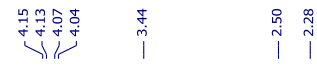
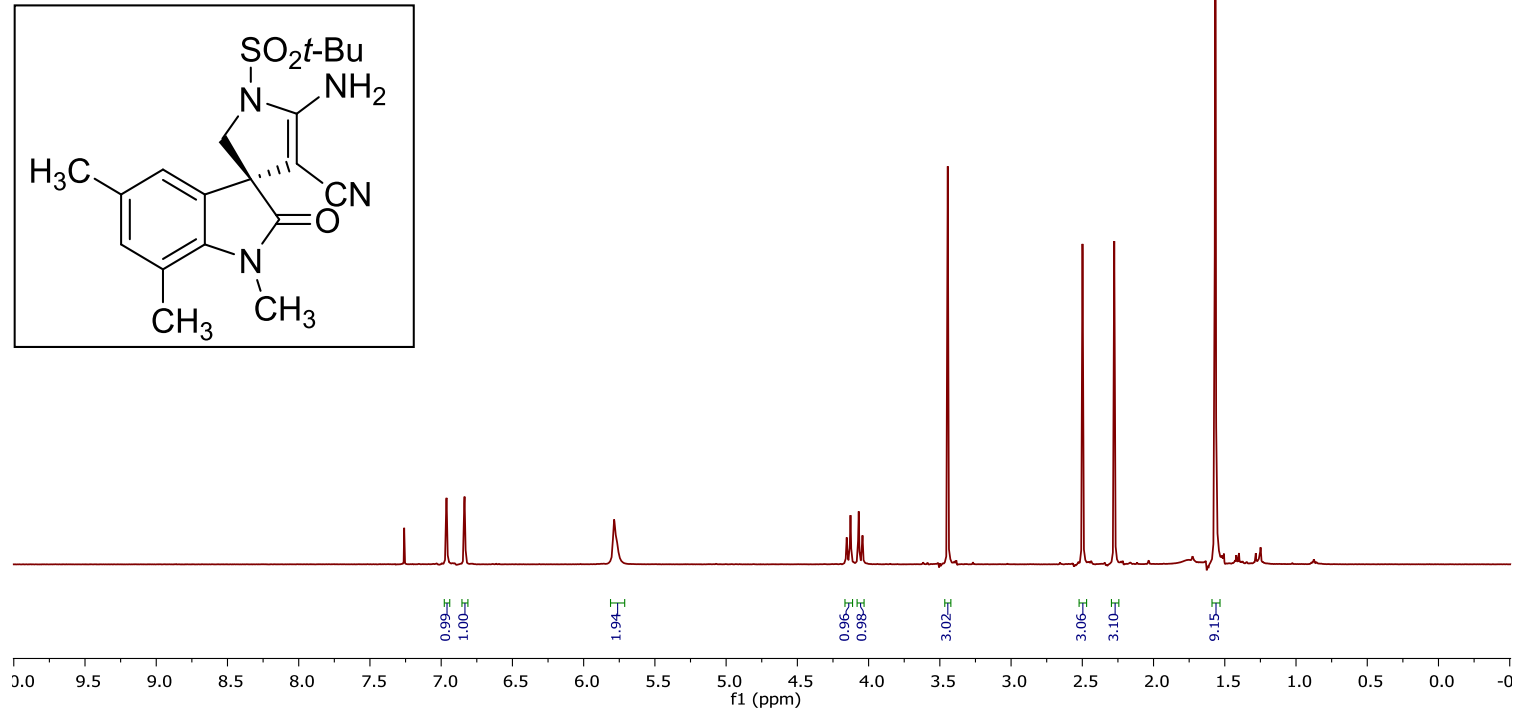

${ }^{1} \mathrm{H}$ NMR of compound 3k (400 $\left.\mathrm{MHz}, \mathrm{CDCl}_{3}\right)$ 


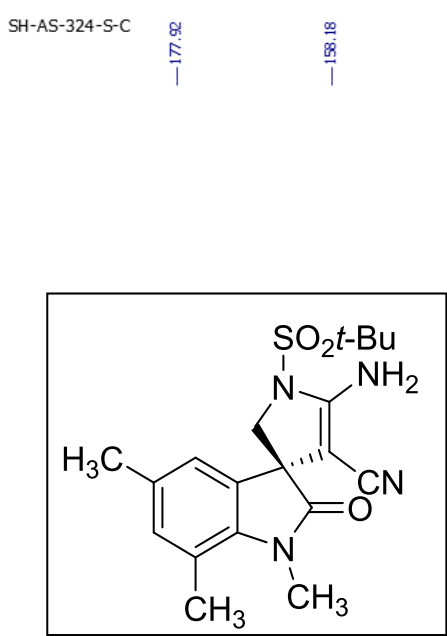

\section{${ }^{13} \mathrm{C}$ NMR of compound 3k (100 $\left.\mathrm{MHz}, \mathrm{CDCl}_{3}\right)$}
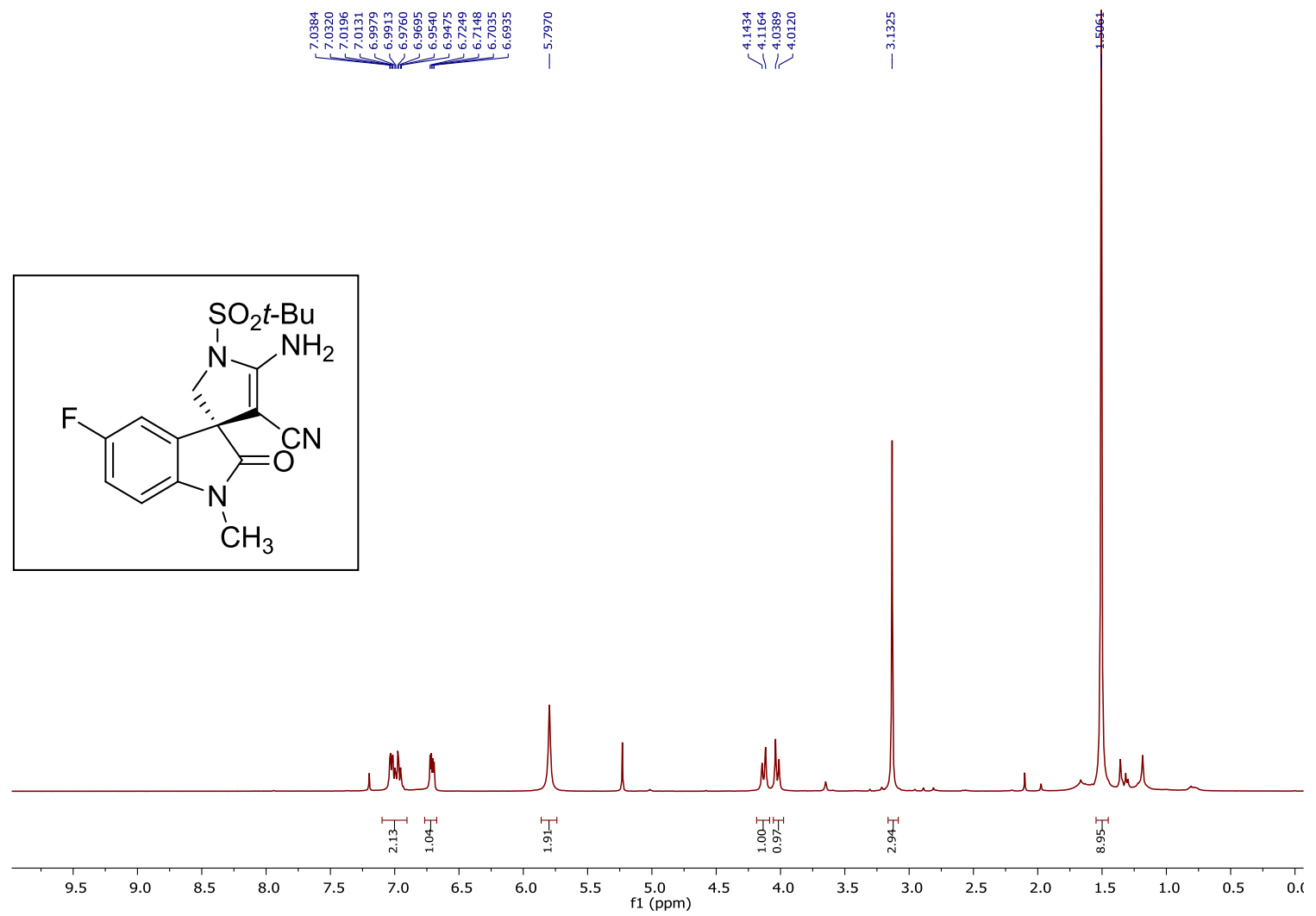

${ }^{1} \mathrm{H}$ NMR of compound ent-31 (400 $\left.\mathrm{MHz}, \mathrm{CDCl}_{3}\right)$ 

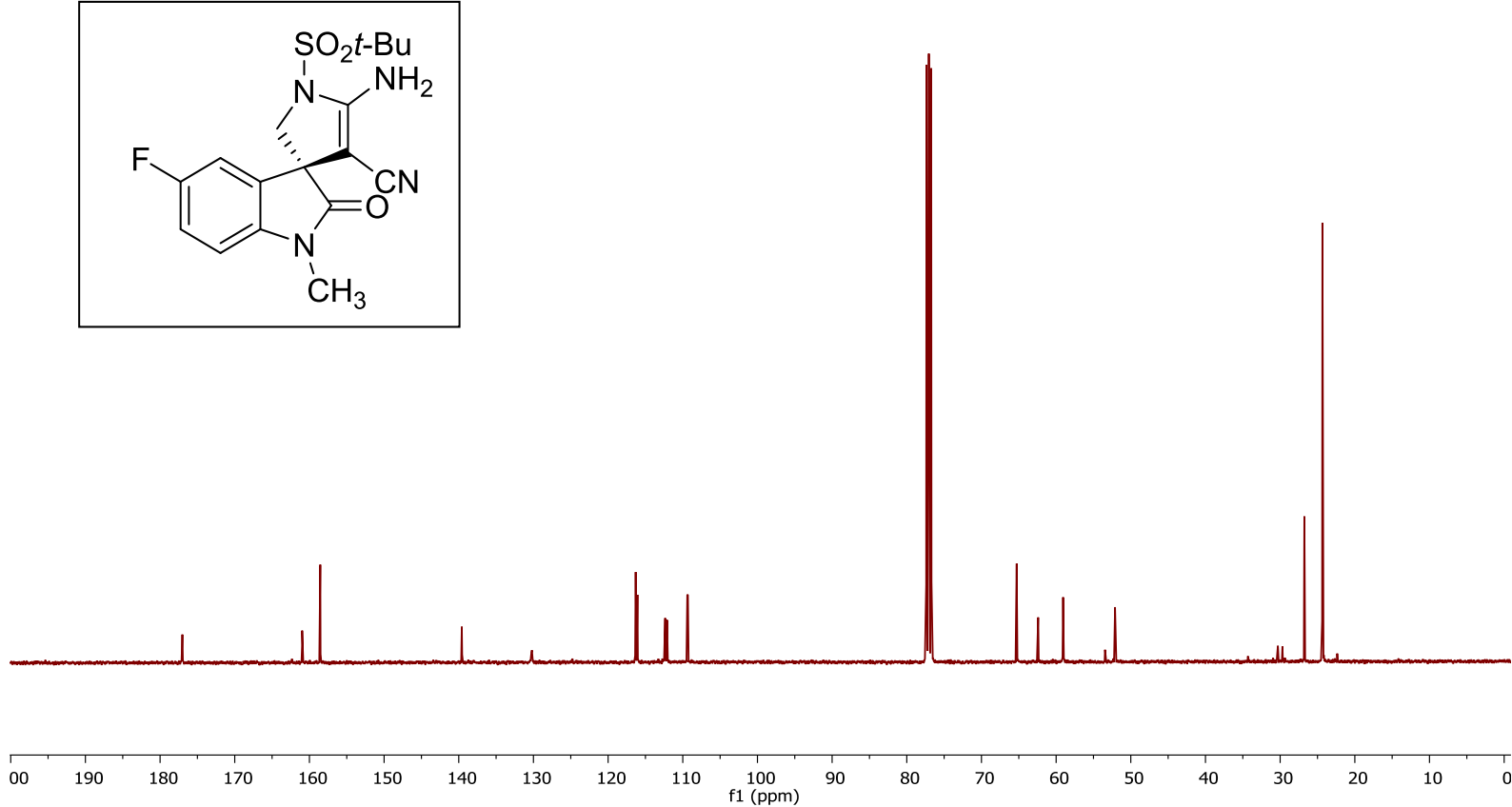

${ }^{13} \mathrm{C}$ NMR of compound ent-31 (100 $\left.\mathrm{MHz}, \mathrm{CDCl}_{3}\right)$

N-Me-5-F-S-MALONO-19F

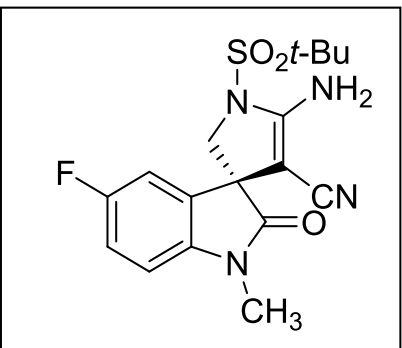

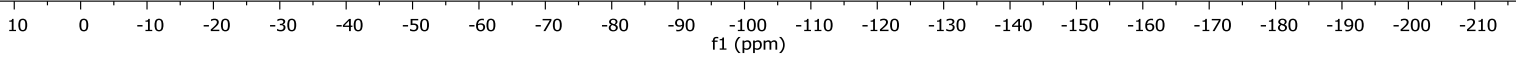
${ }^{19} \mathrm{~F}$ NMR of compound ent-3I (376 $\left.\mathrm{MHz}, \mathrm{CDCl}_{3}\right)$ 


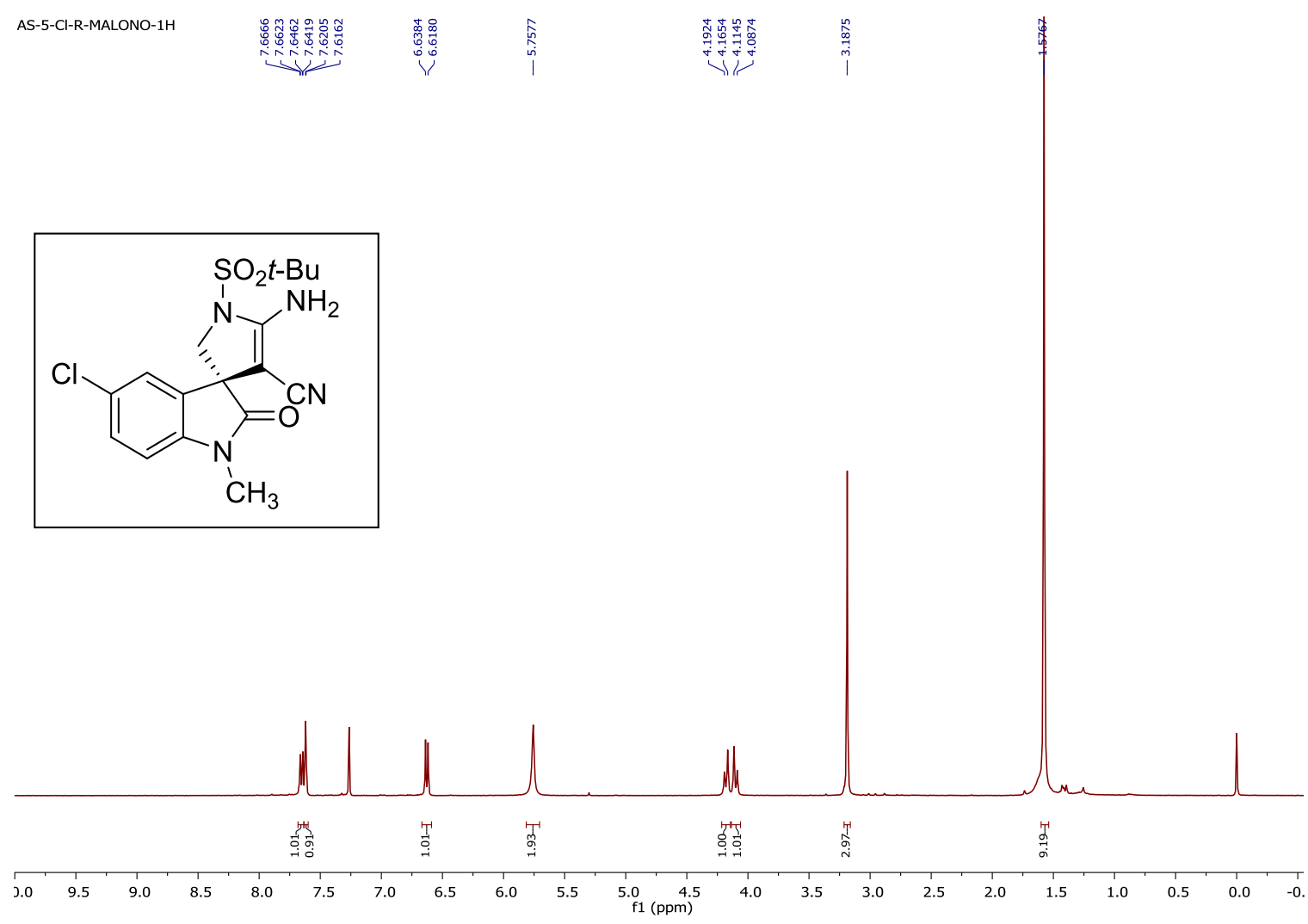

${ }^{1} \mathrm{H}$ NMR of compound ent-3m (400 $\left.\mathrm{MHz}, \mathrm{CDCl}_{3}\right)$
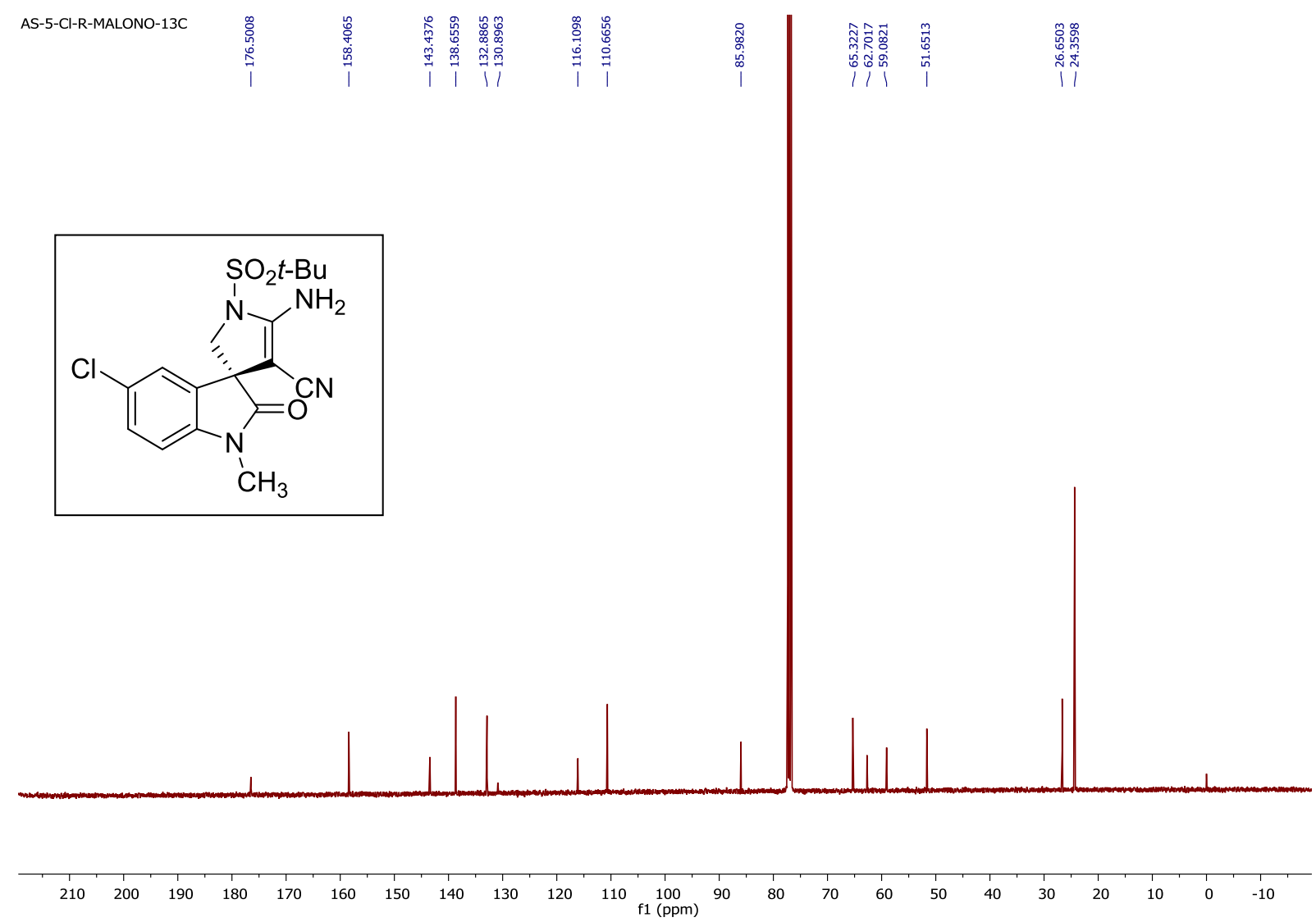

${ }^{13} \mathrm{C}$ NMR of compound ent-3m (100 MHz, $\left.\mathrm{CDCl}_{3}\right)$ 


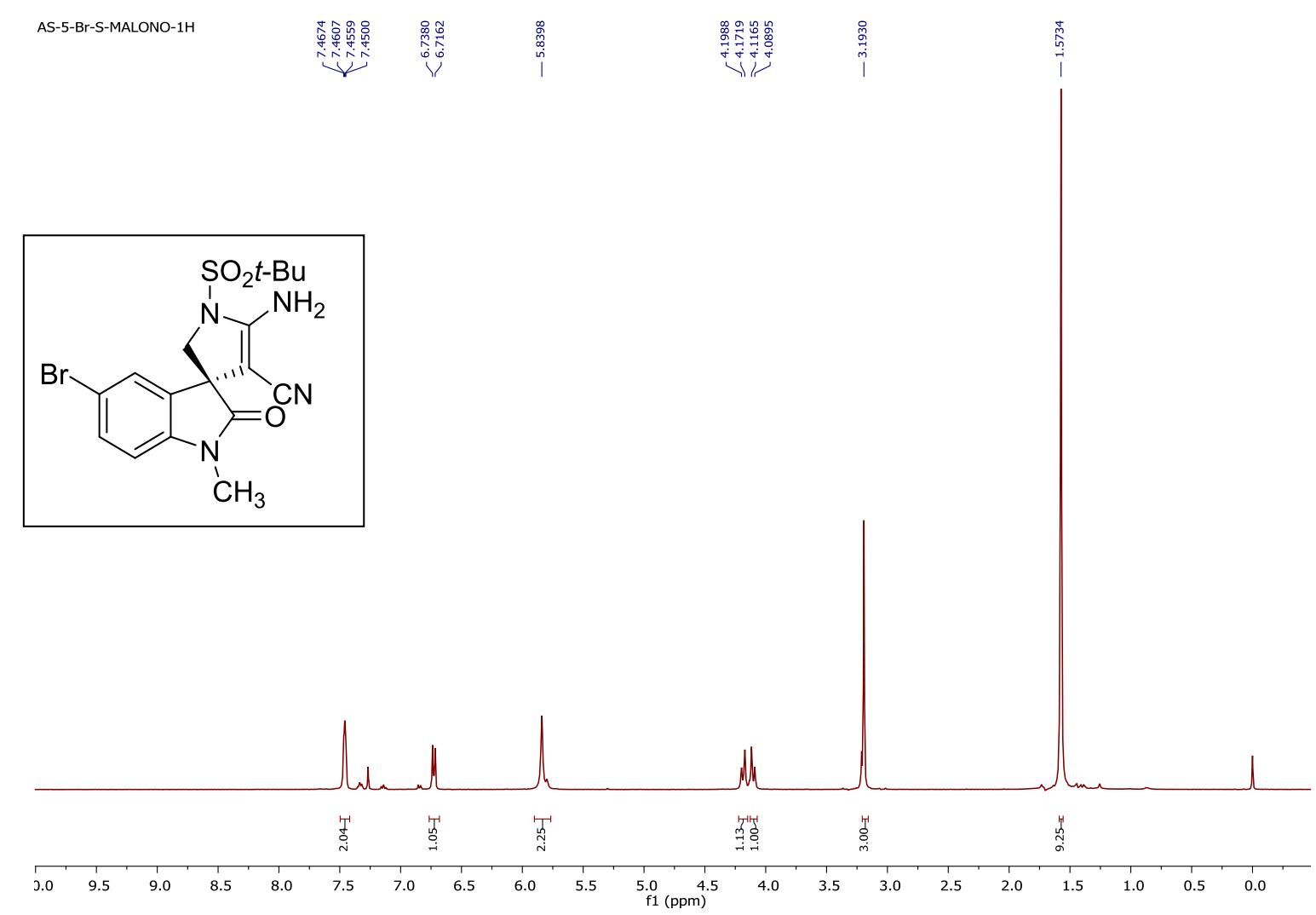

${ }^{1} \mathrm{H}$ NMR of compound 3n (400 $\left.\mathrm{MHz}, \mathrm{CDCl}_{3}\right)$
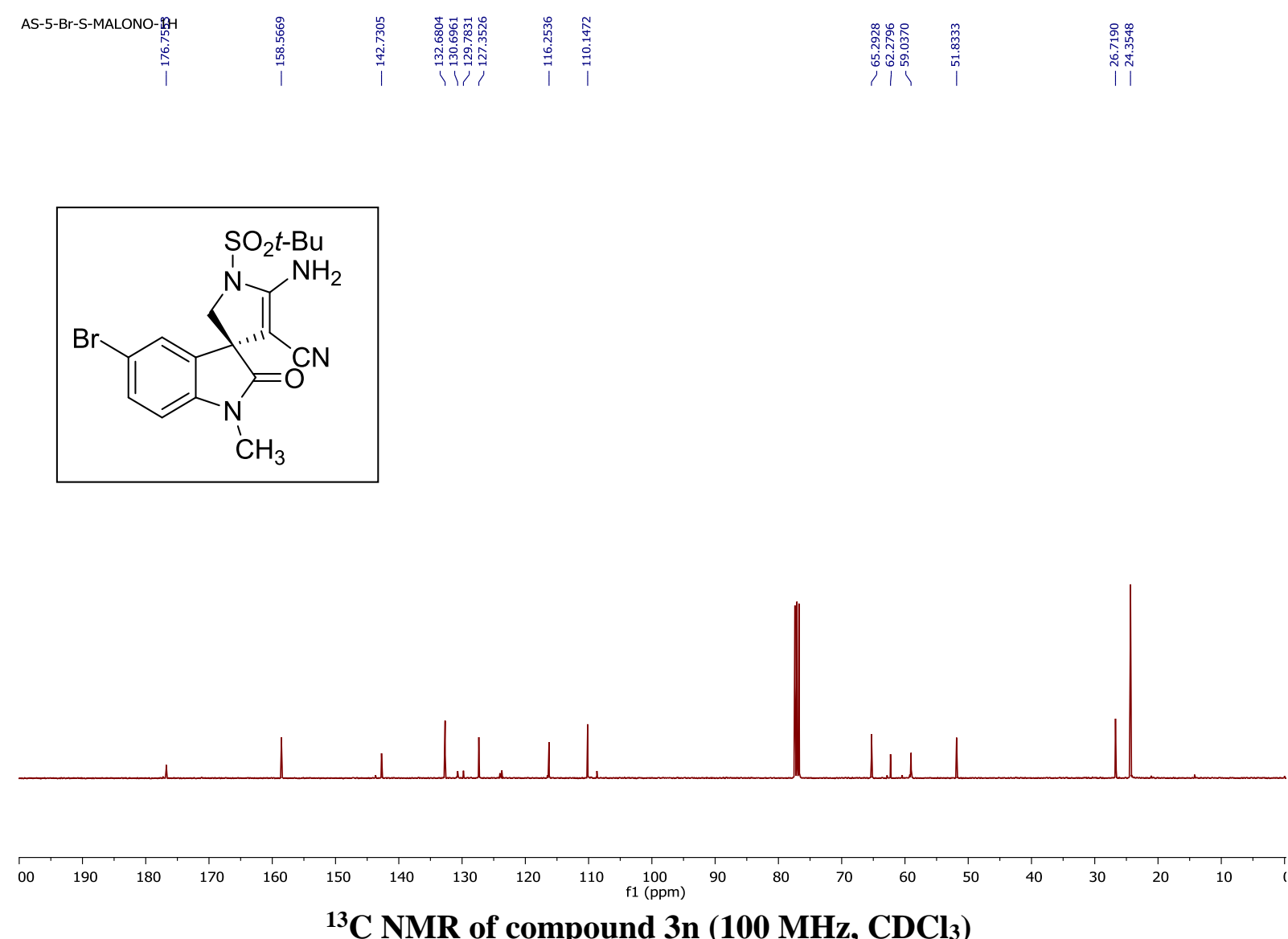

${ }^{13} \mathrm{C}$ NMR of compound 3n (100 $\left.\mathrm{MHz}, \mathrm{CDCl}_{3}\right)$ 


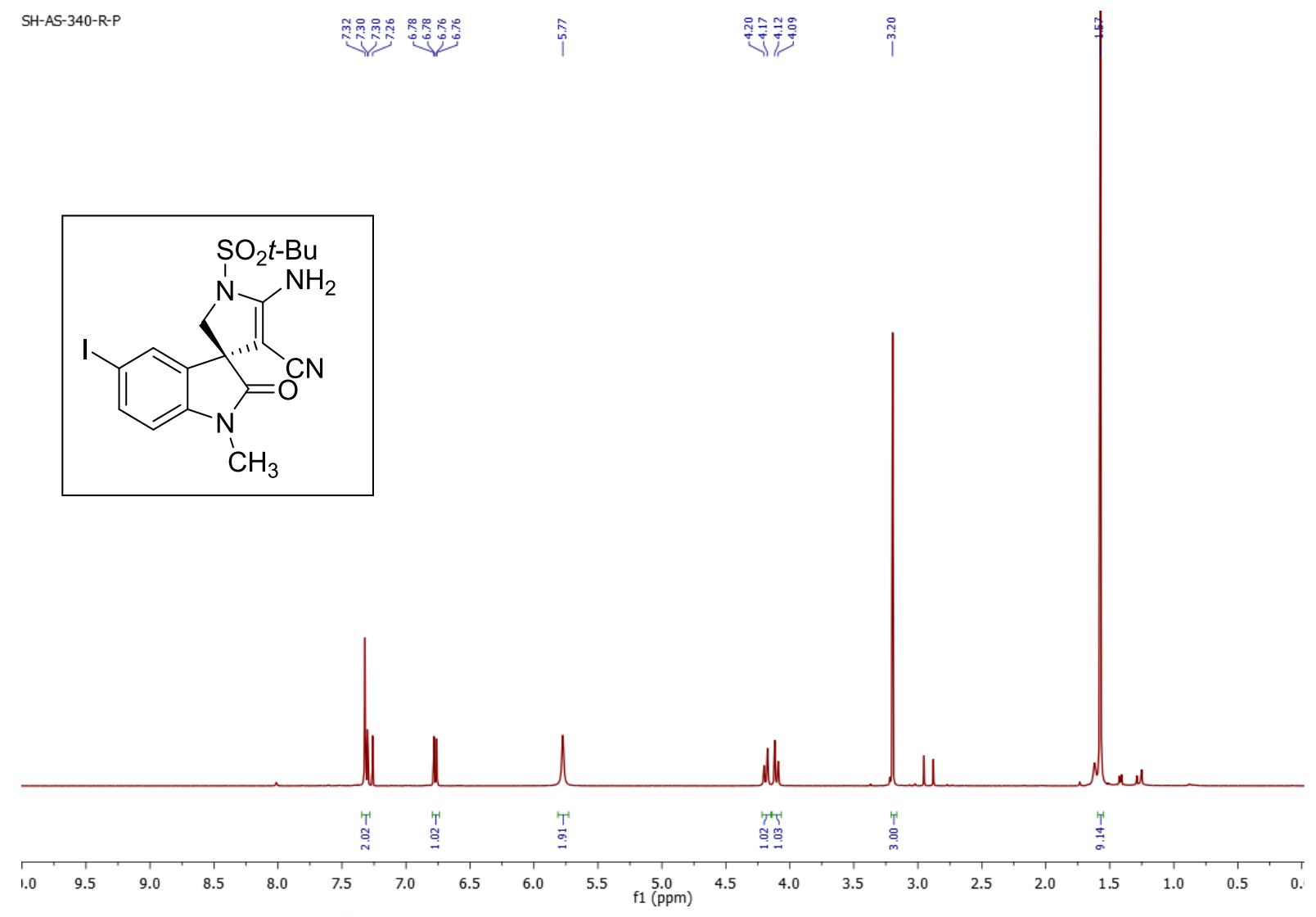

${ }^{1} \mathrm{H}$ NMR of compound 30 (400 $\left.\mathrm{MHz}, \mathrm{CDCl}_{3}\right)$
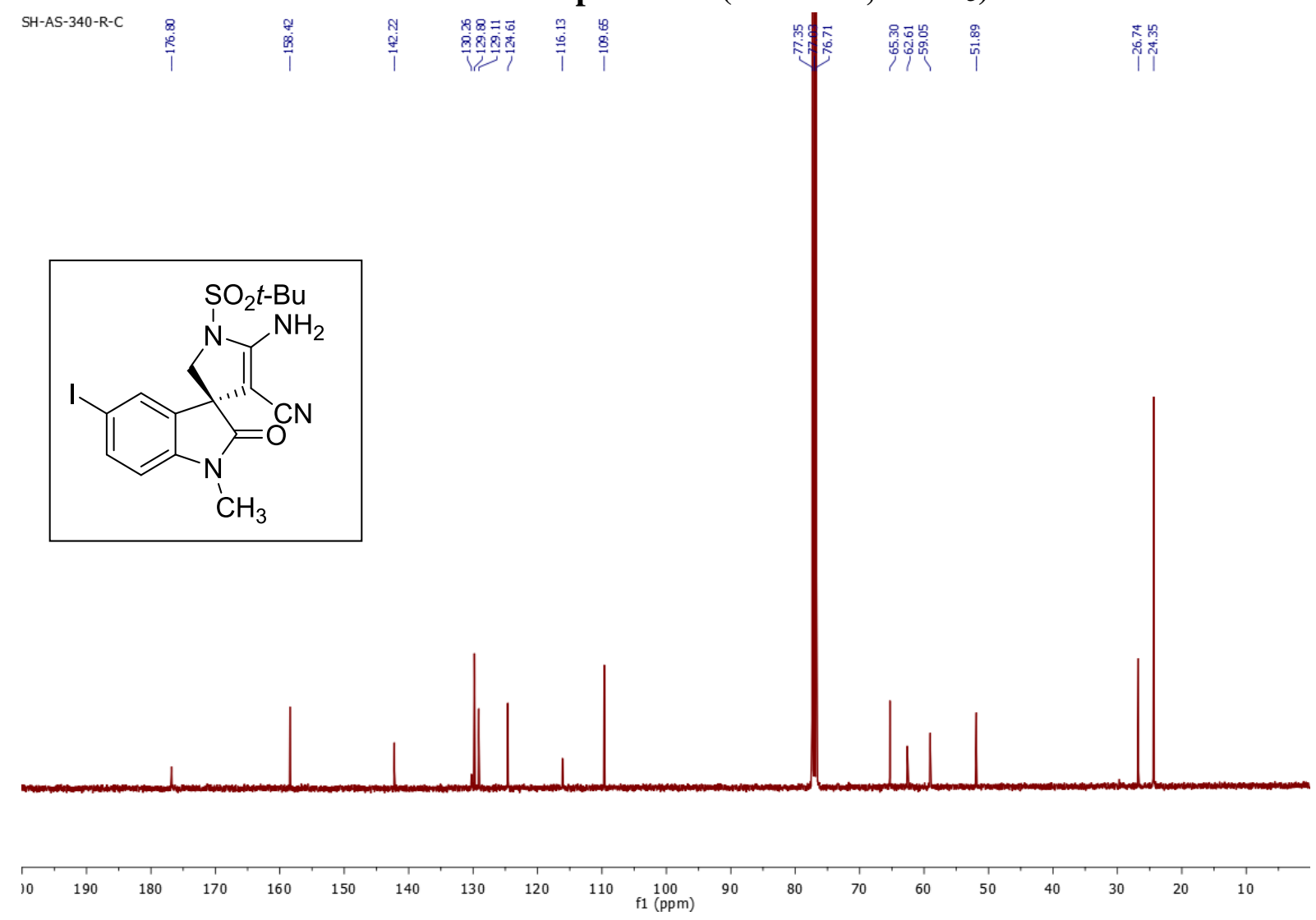

${ }^{13} \mathrm{C}$ NMR of compound $30\left(100 \mathrm{MHz}, \mathrm{CDCl}_{3}\right)$ 

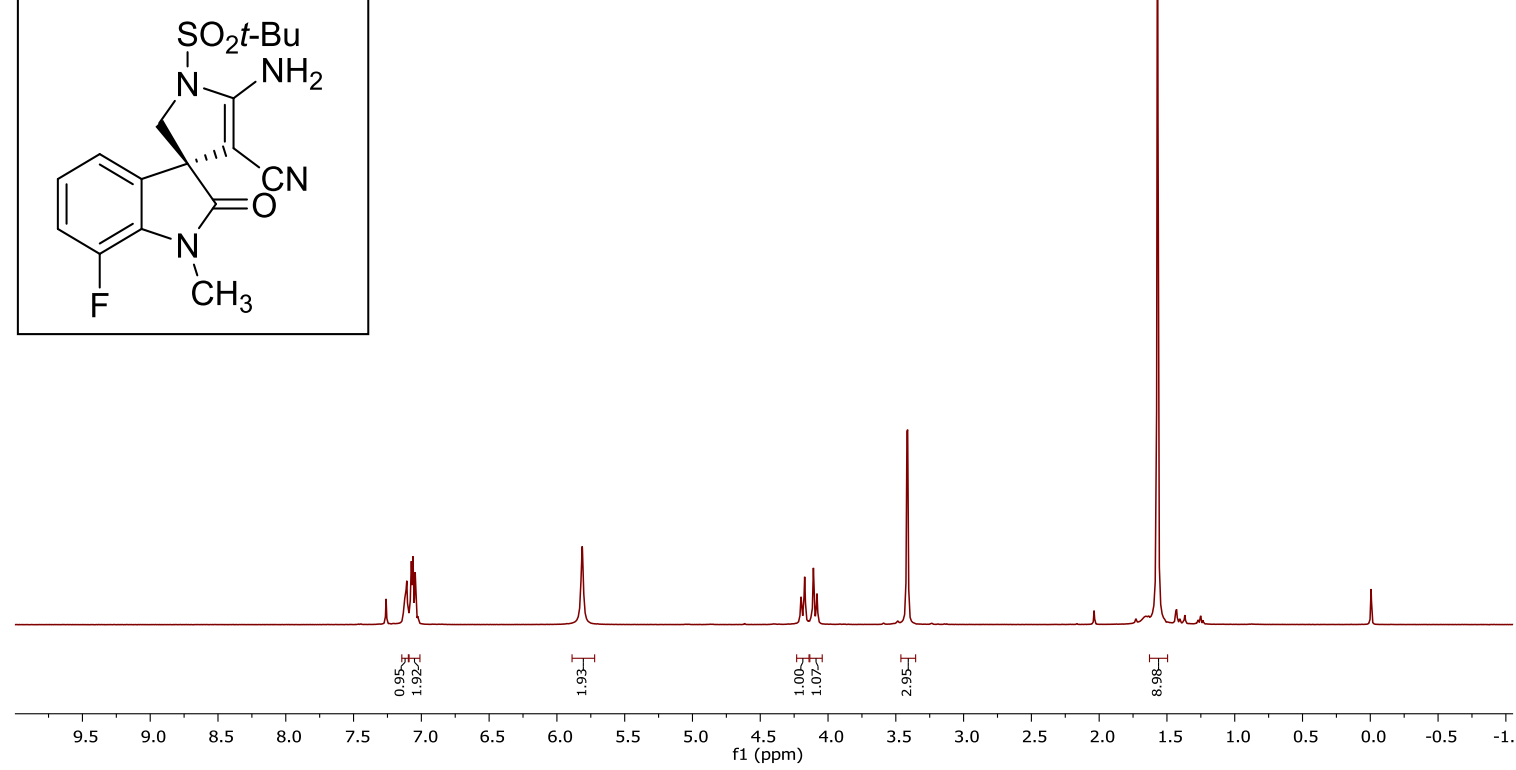

${ }^{1} \mathrm{H}$ NMR of compound 3p (400 $\left.\mathrm{MHz}, \mathrm{CDCl}_{3}\right)$

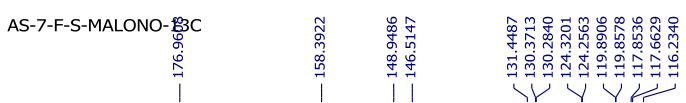
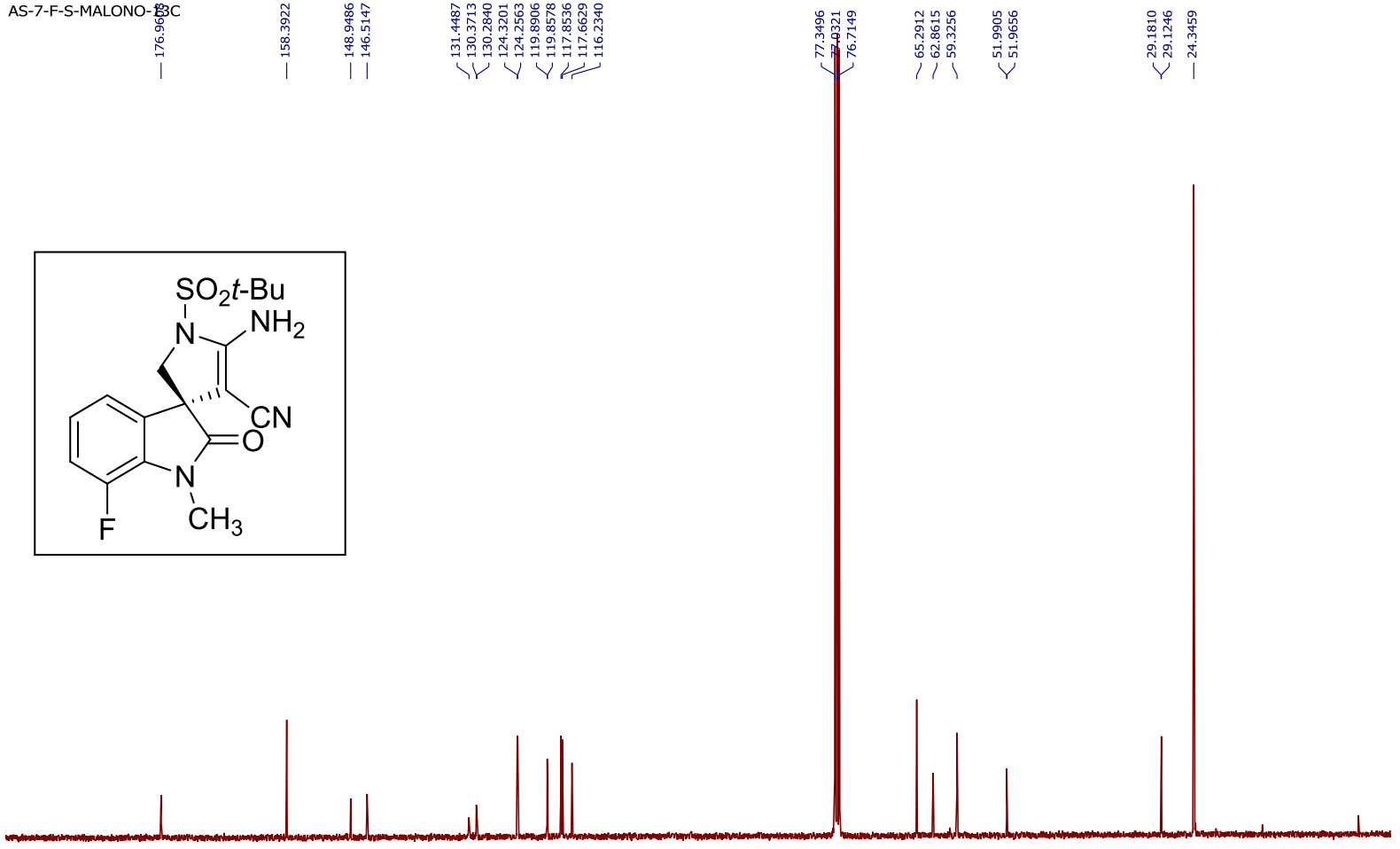

${ }^{13} \mathrm{C}$ NMR of compound 3p (100 MHz, $\left.\mathrm{CDCl}_{3}\right)$ 

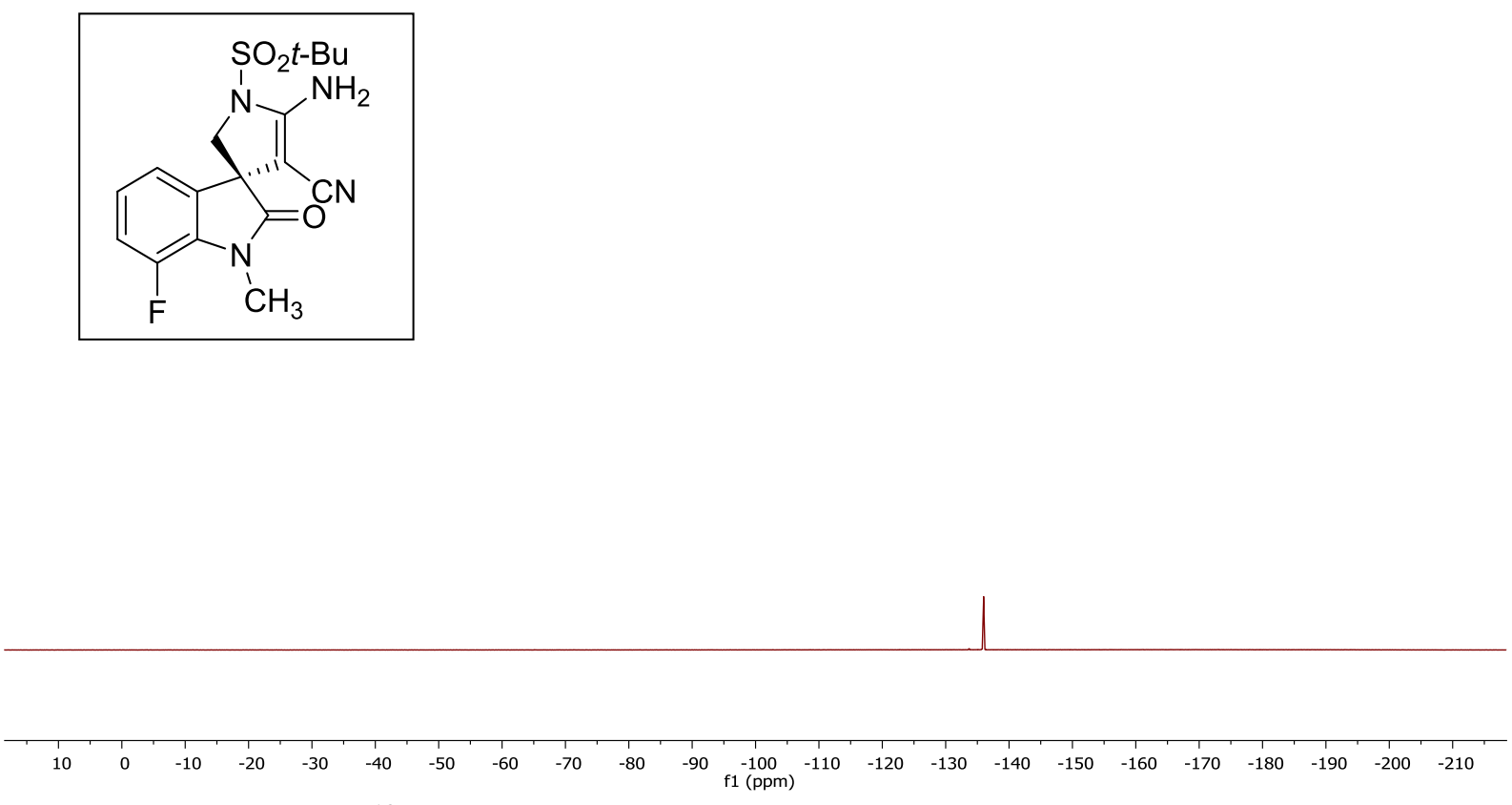

${ }^{19}$ F NMR of compound 3p (376 $\left.\mathrm{MHz}, \mathrm{CDCl}_{3}\right)$

AS-7-CI-MALONO-1H

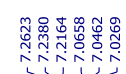

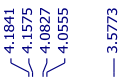
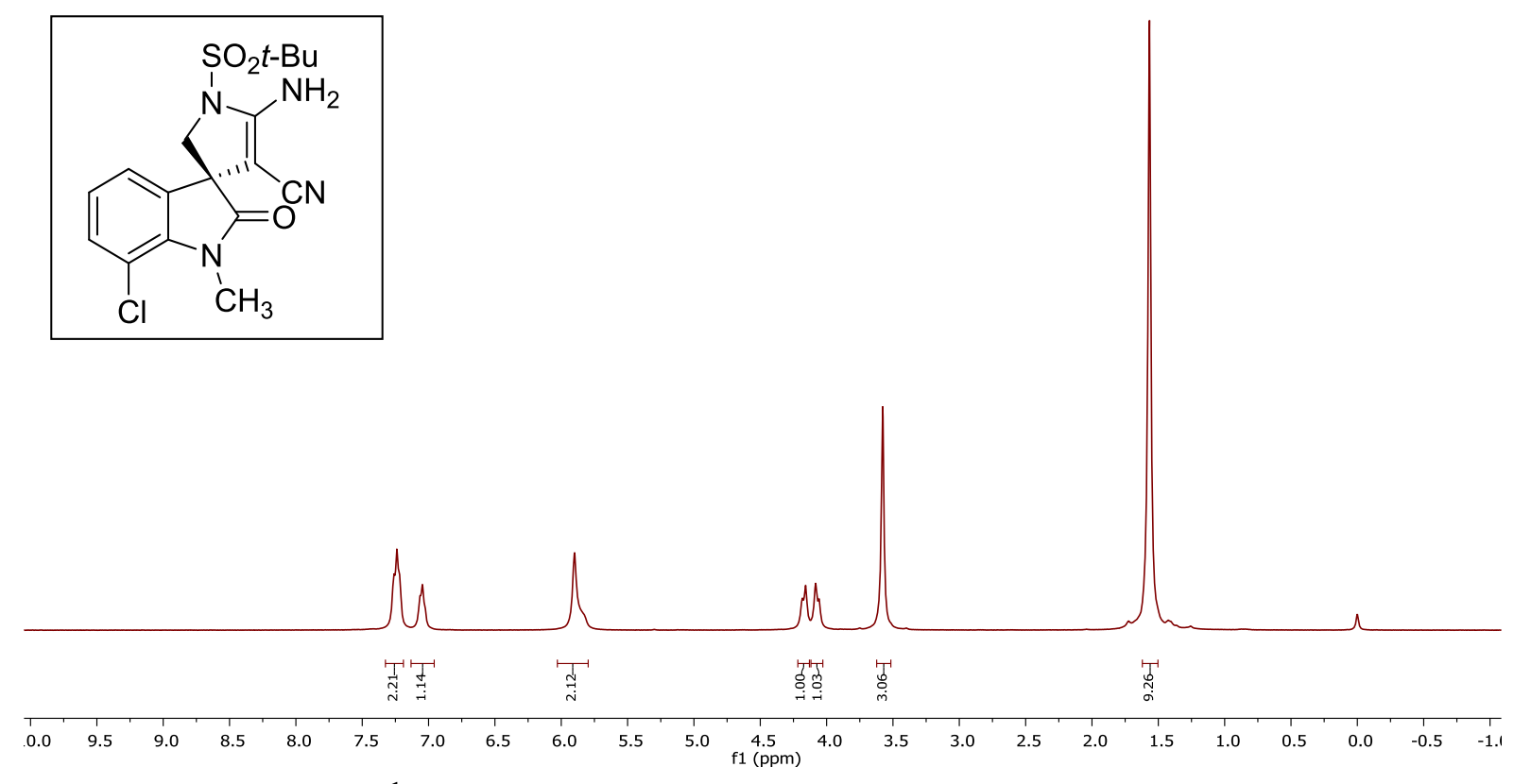

${ }^{1} \mathrm{H}$ NMR of compound $3 q\left(400 \mathrm{MHz}, \mathrm{CDCl}_{3}\right)$ 


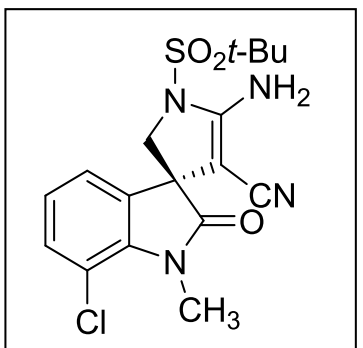

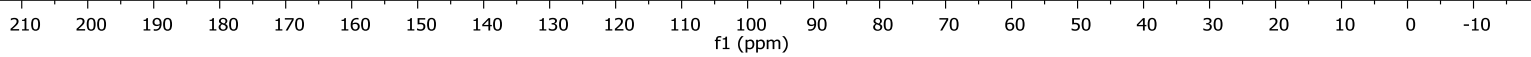
${ }^{13} \mathrm{C}$ NMR of compound 3q (100 $\left.\mathrm{MHz}, \mathrm{CDCl}_{3}\right)$

AS-7-Br-S-MALONO-1H

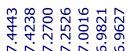
商

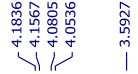<smiles>CN1C(=O)[C@@]2(CN1S(=O)(=O)O[Na])C(C#N)=C(N)N2C</smiles>
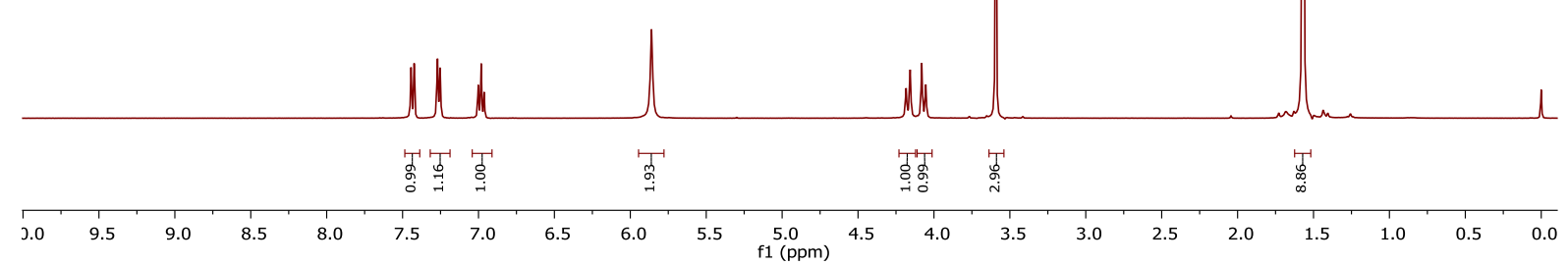

${ }^{1} \mathrm{H}$ NMR of compound $3 \mathbf{r}\left(400 \mathrm{MHz}, \mathrm{CDCl}_{3}\right)$ 

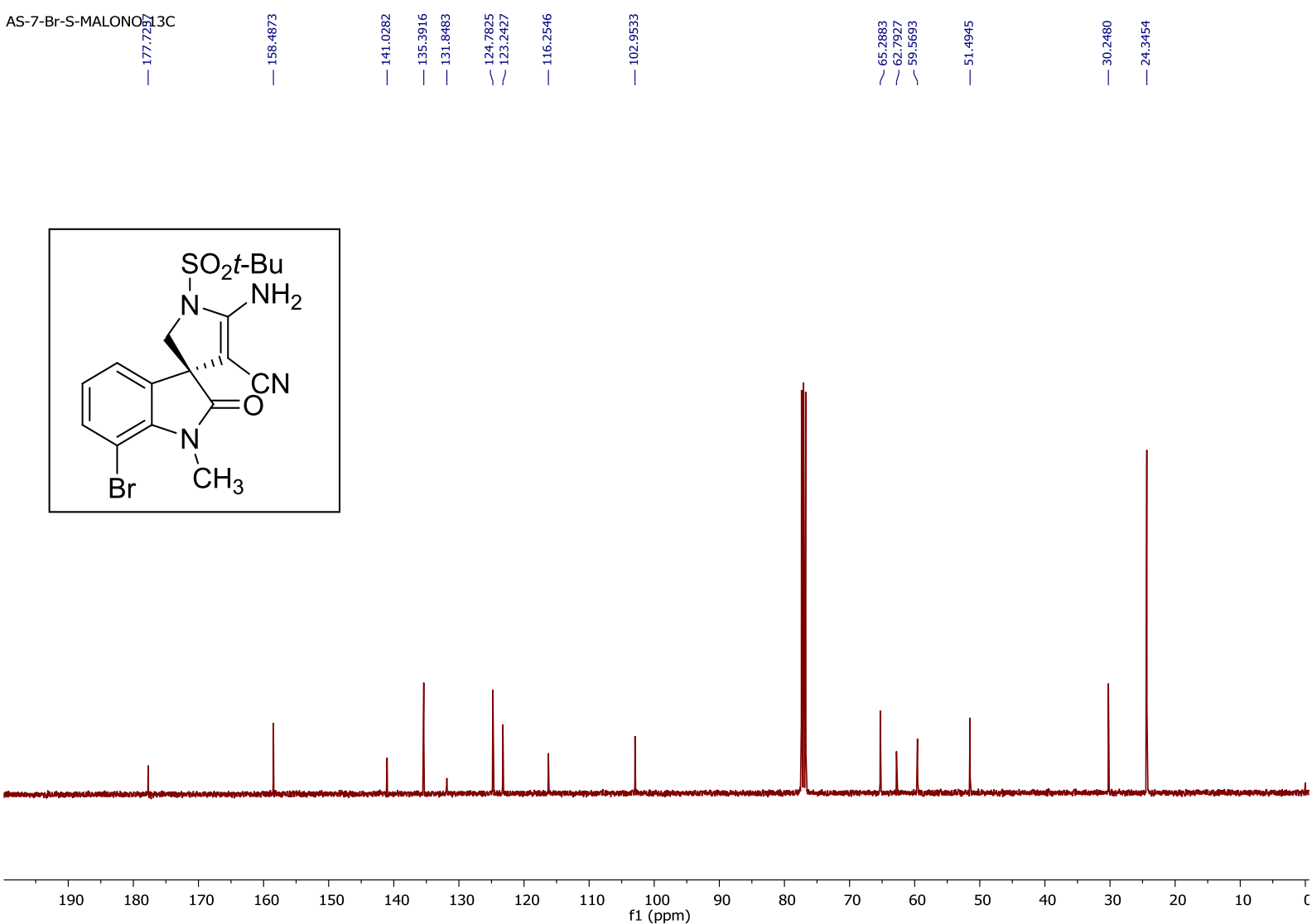

${ }^{13} \mathrm{C}$ NMR of compound $3 \mathrm{r}\left(100 \mathrm{MHz}, \mathrm{CDCl}_{3}\right)$
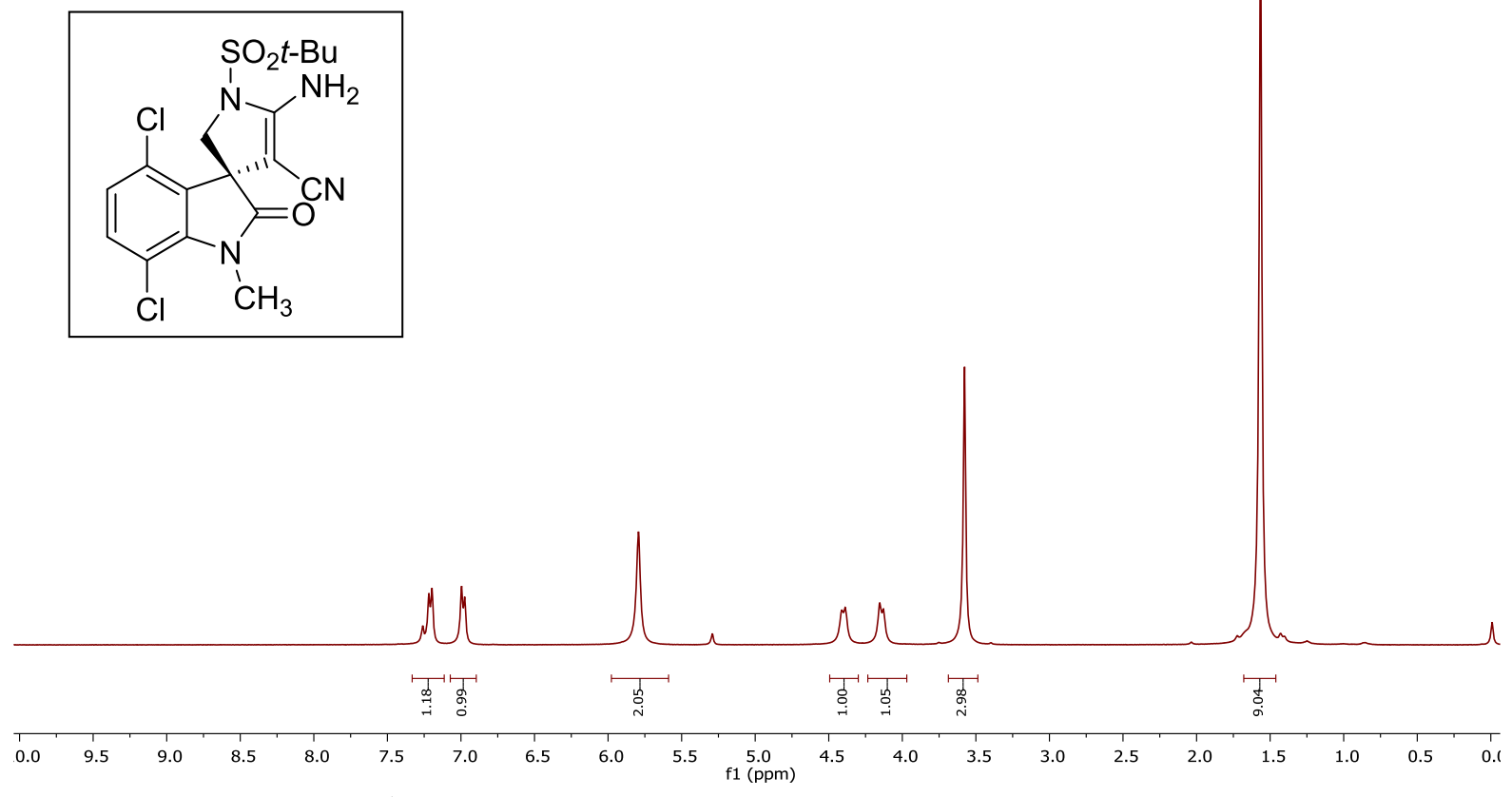

${ }^{1} \mathrm{H}$ NMR of compound 3s (400 MHz, $\left.\mathrm{CDCl}_{3}\right)$ 

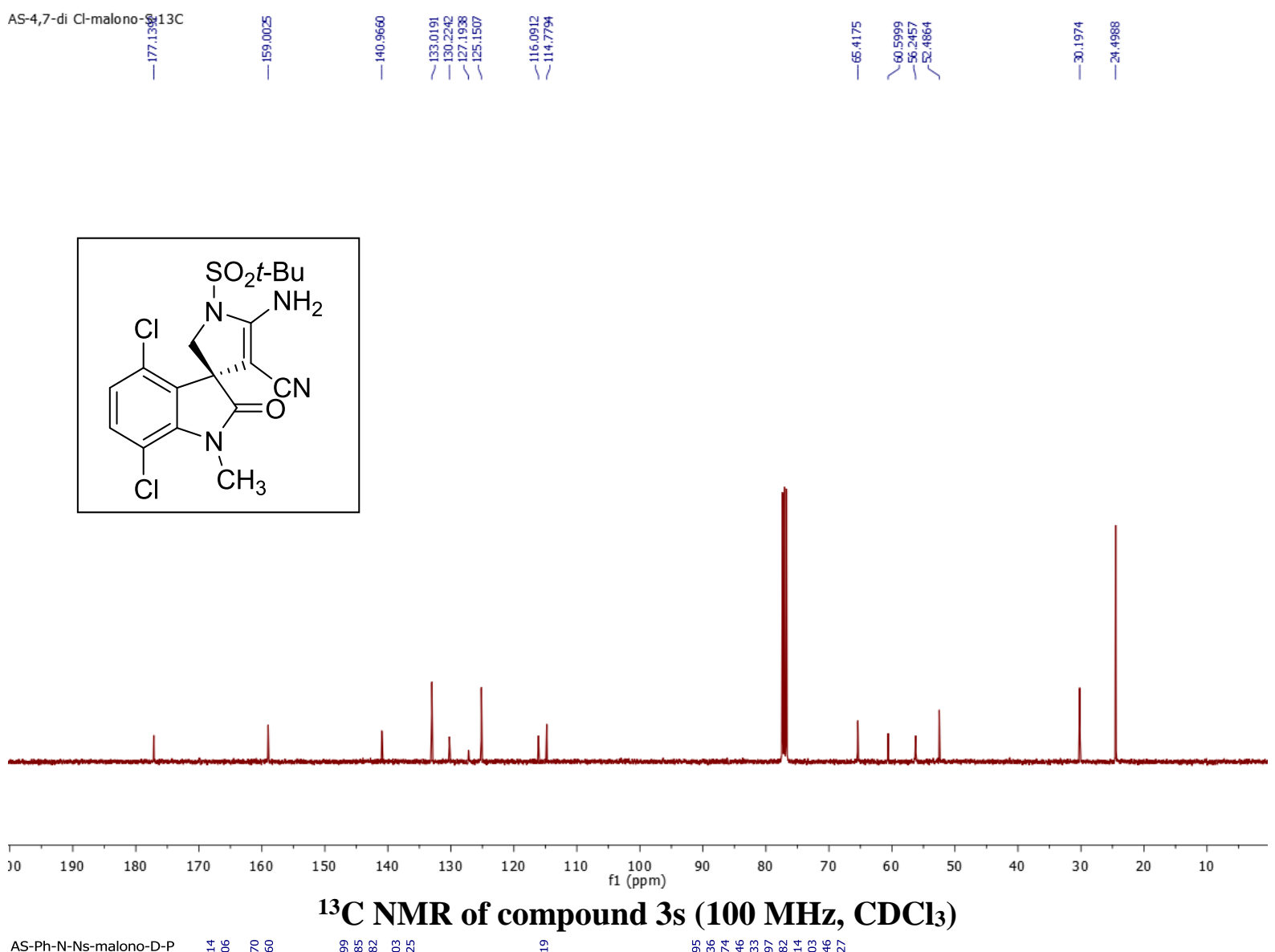

AS-Ph-N-Ns-malono-D-P
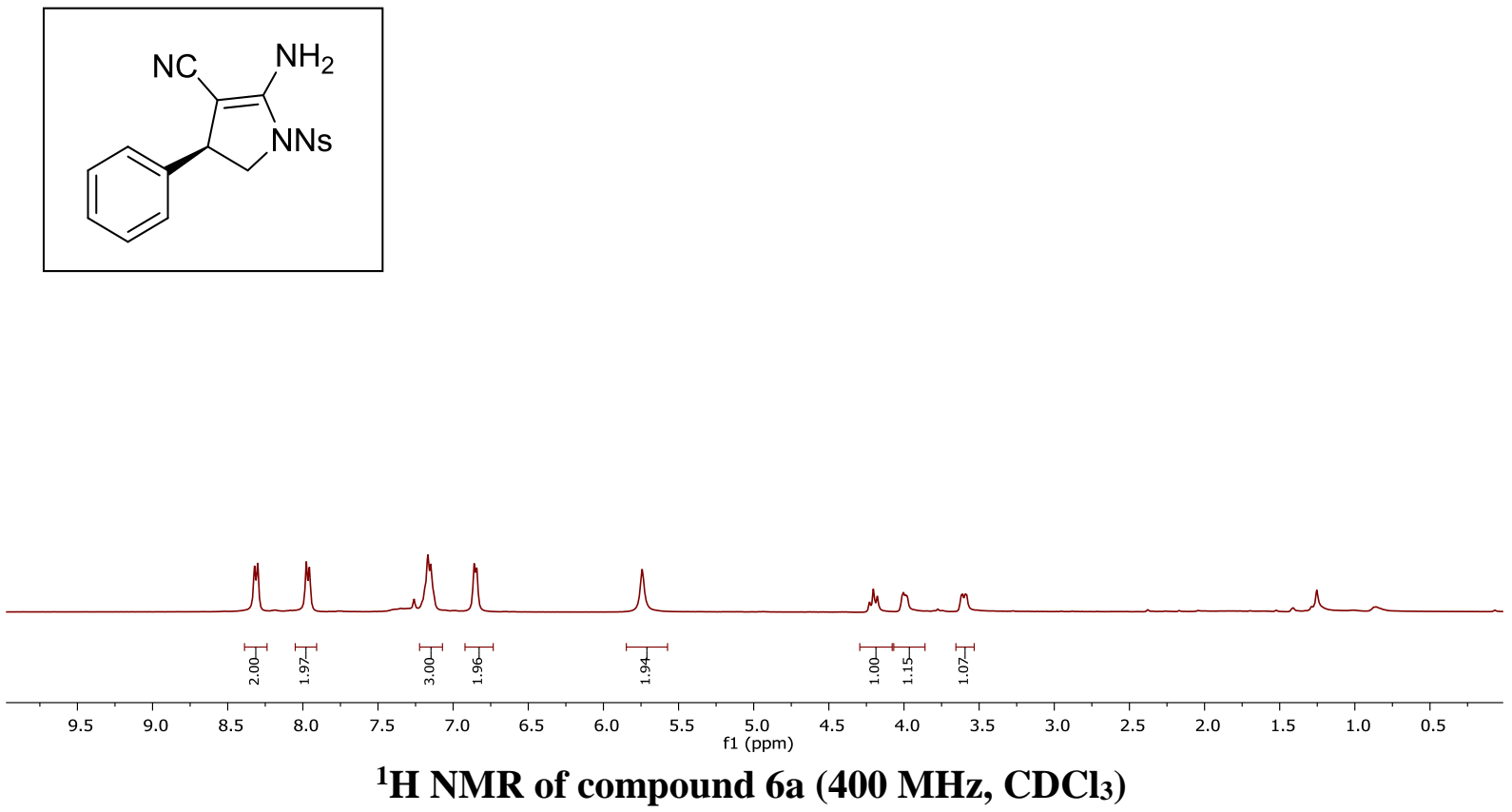

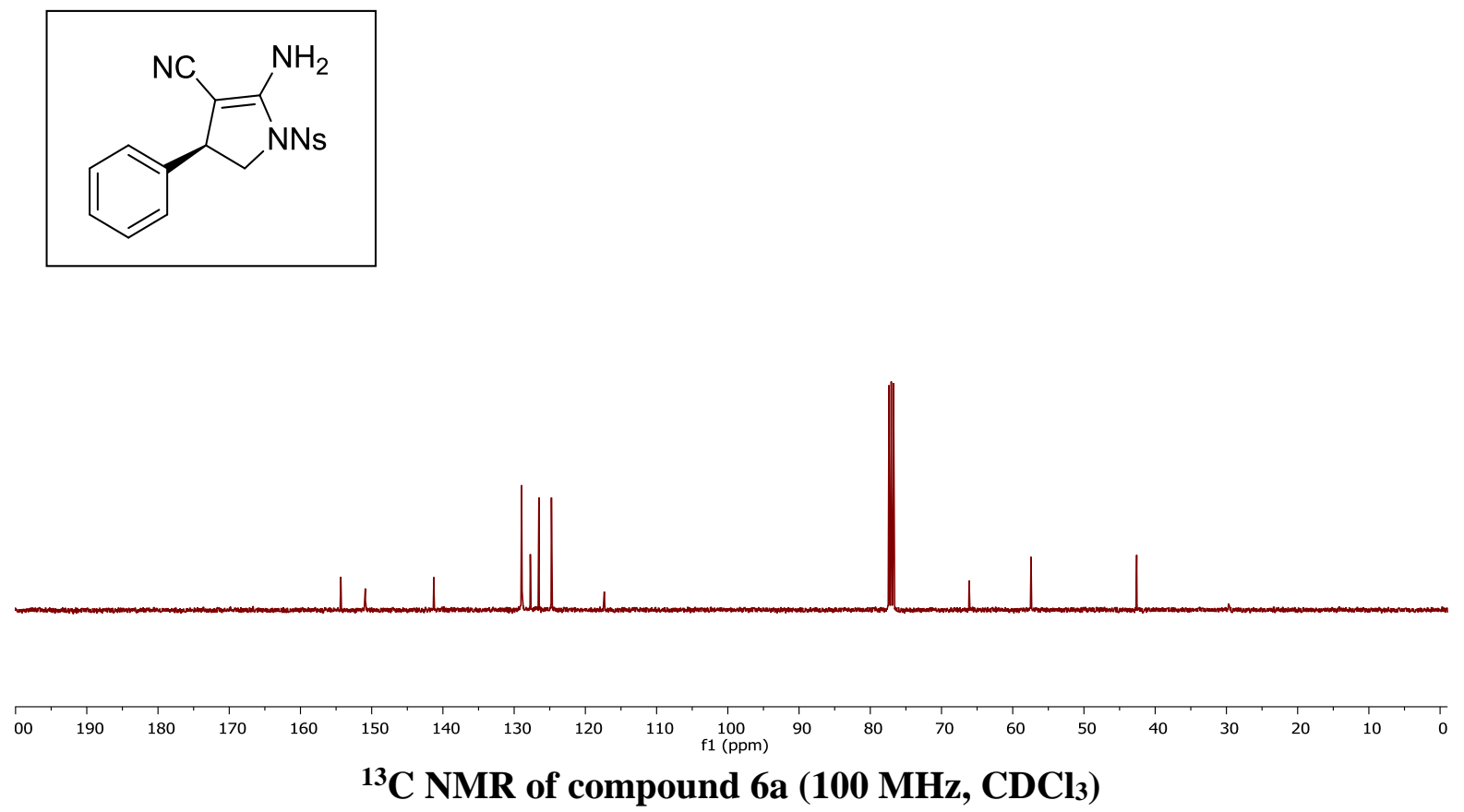

Phenyl-N-Ts-L-malono-1H
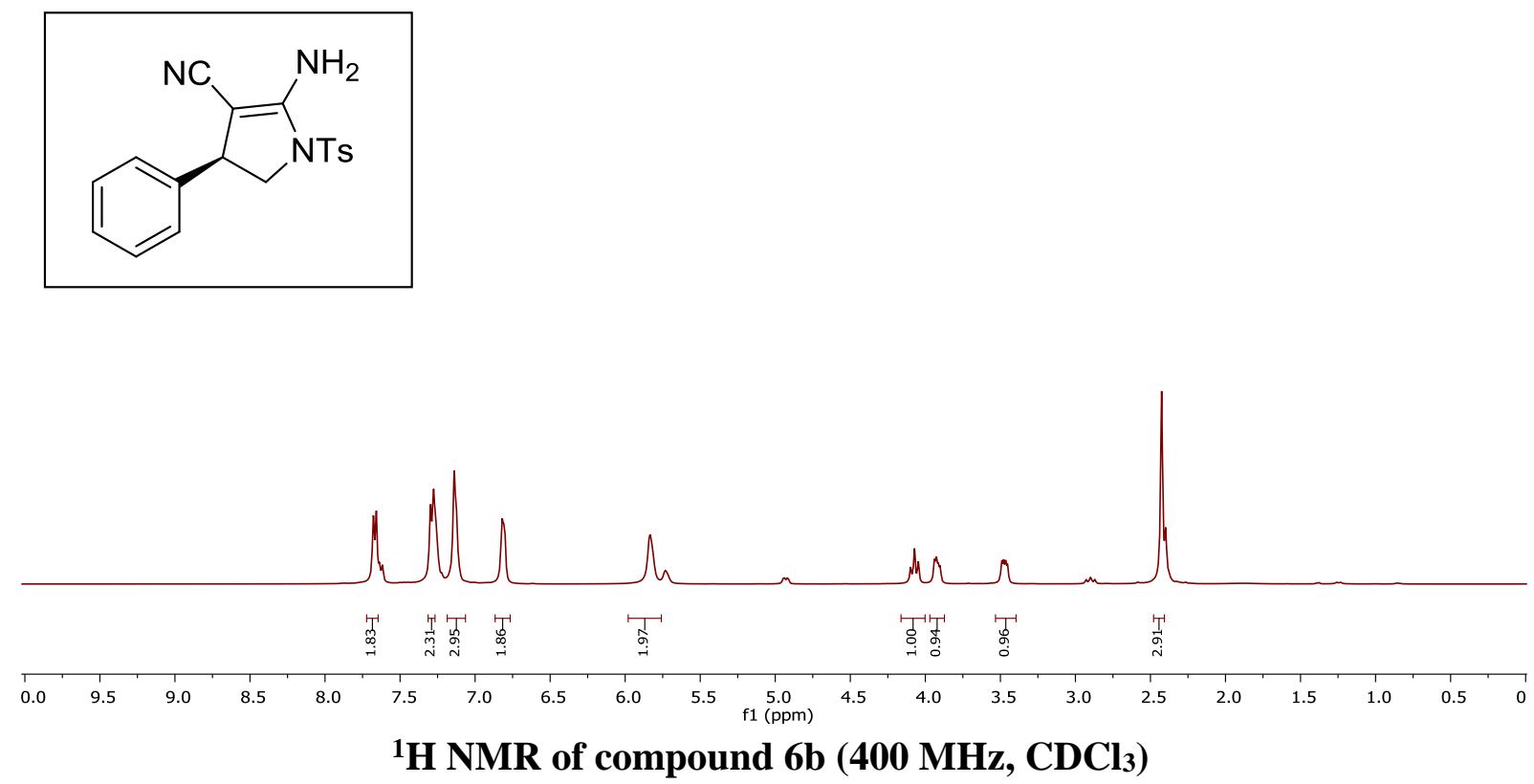

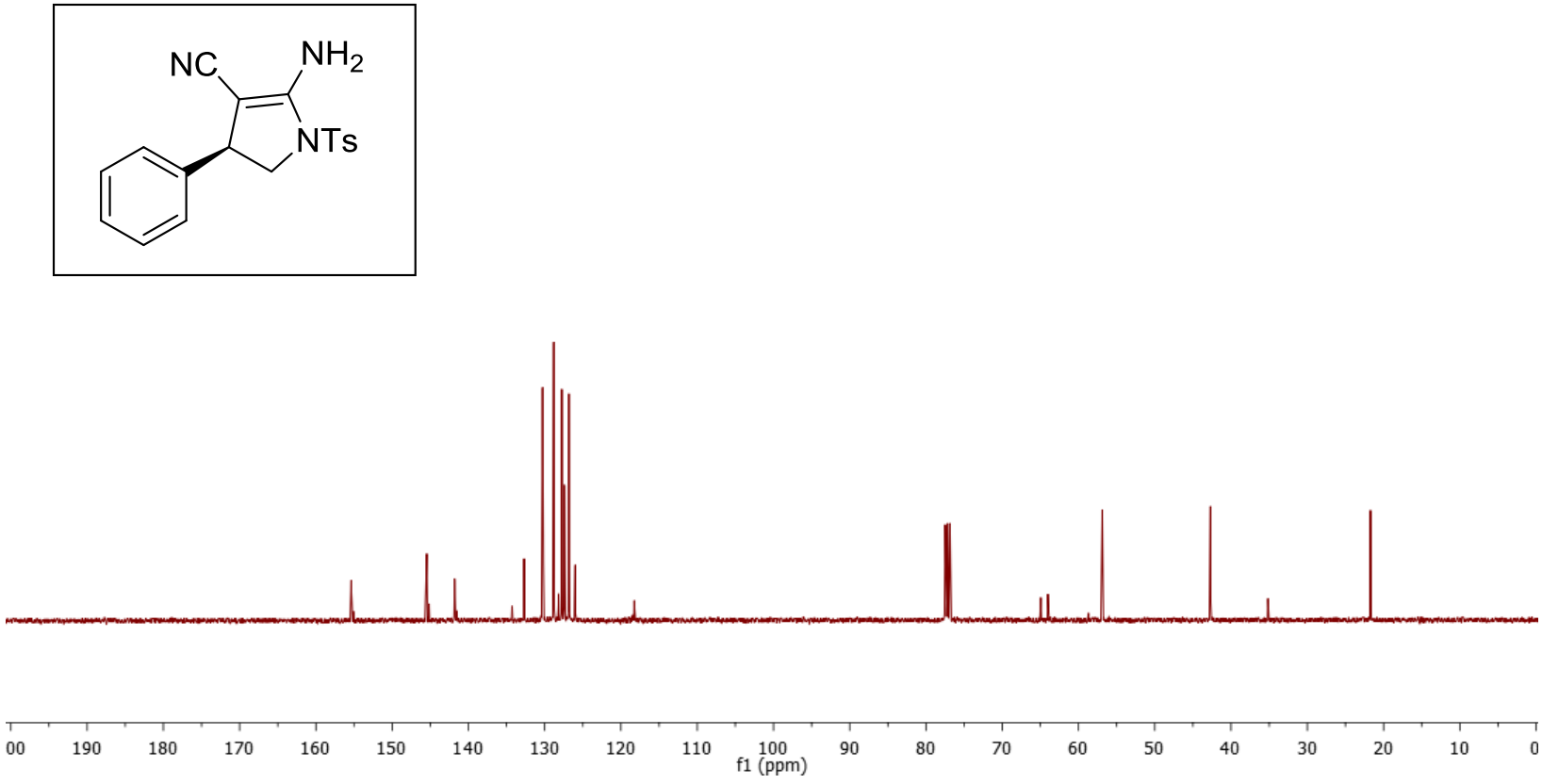

${ }^{13} \mathrm{C}$ NMR of compound $6 \mathrm{~b}\left(100 \mathrm{MHz}, \mathrm{CDCl}_{3}\right)$

\section{X-ray crystal structure details:}

\section{Datablock for the compound ent-3m:}

The details of crystal data collection and refinement of $\boldsymbol{e n t - 3 m}$ are summarized in Table S1. CCDC 1918606 contains the supplementary crystallographic data for this paper. This data can be obtained free of charge from The Cambridge Crystallography Data Center via www.ccdc.cam.ac.uk/data_request/cif. 


\begin{tabular}{|c|c|c|c|}
\hline Bond precision: & $C-C=0.0057$ & A & Wavelength $=0.71073$ \\
\hline \multirow[t]{2}{*}{ Cell: } & $a=8.8449(4)$ & & $\mathrm{b}=18.7623(8)$ \\
\hline & alpha $=90$ & & beta $=95.801(1)$ \\
\hline \multirow[t]{2}{*}{ Temperature: } & $273 \mathrm{~K}$ & & \\
\hline & Calculated & & Reported \\
\hline Volume & $1876.60(14)$ & & $1876.60(14)$ \\
\hline Space group & P 21 & & $\begin{array}{llll}P & 1 & 21 & 1\end{array}$ \\
\hline Hall group & P 2yb & & P 2yb \\
\hline Moiety formula & $\mathrm{C} 17 \mathrm{H} 19 \mathrm{Cl} \mathrm{N} 4$ & 03 & $2(\mathrm{C} 17 \mathrm{H} 19 \mathrm{Cl}$ N4 $\mathrm{O} 3 \mathrm{~S})$ \\
\hline Sum formula & C17 H19 Cl N4 & 03 & C34 H38 C12 N8 O6 S2 \\
\hline Mr & 394.87 & & 789.74 \\
\hline $\mathrm{Dx}, \mathrm{g} \mathrm{cm}-3$ & 1.398 & & 1.398 \\
\hline $\mathrm{Z}$ & 4 & & 2 \\
\hline $\mathrm{Mu} \quad(\mathrm{mm}-1)$ & 0.340 & & 0.340 \\
\hline $\mathrm{F} 000$ & 824.0 & & 824.0 \\
\hline F000' & 825.39 & & \\
\hline $\mathrm{h}, \mathrm{k}, \mathrm{lmax}$ & $10,22,13$ & & $10,22,13$ \\
\hline Nref & $6625[3423]$ & & 6504 \\
\hline Tmin, Tmax & $0.906,0.934$ & & $0.643,0.746$ \\
\hline Tmin' & 0.906 & & \\
\hline
\end{tabular}

Correction method $=\#$ Reported $\mathrm{T}$ Limits: $\mathrm{Tmin}=0.643 \mathrm{Tmax}=0.746$ AbsCorr = MULTI-SCAN

Data completeness $=1.90 / 0.98 \quad$ Theta $(\max )=25.000$

$\mathrm{R}($ reflections $)=0.0339(6426) \quad$ wR2 (reflections $)=0.0890(6504)$

$S=1.048 \quad$ Npar $=493$

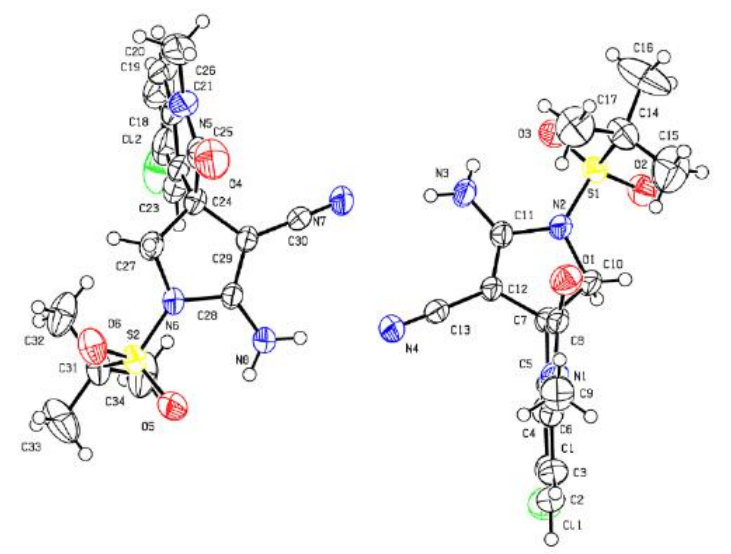

ORTEP diagram of $\boldsymbol{e n t}$-3m 


\section{HPLC data:}

Sample Info

N-Me-MALONO-MIX

CHIRALPAK IA-3; 250 MM

IPA/HEXANE : $15 / 85$

FLOW RATE: $1.0 \mathrm{~mL} / \mathrm{min}$

$254 \mathrm{~nm}$

Additional Info : Peak(s) manually integrated

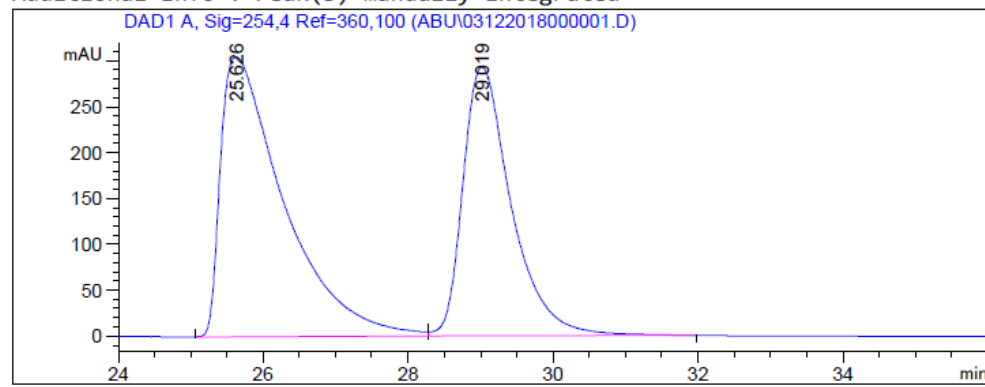

Area Percent Report

\begin{tabular}{|c|c|c|c|c|}
\hline Sorted By & : & Signal & & \\
\hline Multiplier & : & 1.0000 & & \\
\hline Dilution & $:$ & 1.0000 & & \\
\hline \multicolumn{5}{|c|}{ Use Multiplier \& Dilution Factor with ISTDs } \\
\hline \multicolumn{5}{|c|}{ Signal 1: DAD1 A, Sig=254,4 Ref=360,100 } \\
\hline $\begin{array}{l}\text { Peak RetTime Type } \\
\# \text { [min] }\end{array}$ & $\begin{array}{l}\text { Width } \\
\text { [min] }\end{array}$ & $\begin{array}{c}\text { Area } \\
{[\mathrm{mAU} * \mathrm{~s}]}\end{array}$ & $\begin{array}{l}\text { Height } \\
\text { [mAU] }\end{array}$ & $\begin{array}{c}\text { Area } \\
\%\end{array}$ \\
\hline $\begin{array}{ll}1 & 25.626 \mathrm{BV} \\
2 & 29.019 \mathrm{VB}\end{array}$ & $\begin{array}{l}0.8478 \\
0.6959\end{array}$ & $\begin{array}{l}1.85099 \mathrm{e} 4 \\
1.36266 \mathrm{e} 4\end{array}$ & $\begin{array}{l}305.10190 \\
293.34335\end{array}$ & $\begin{array}{l}57.5978 \\
42.4022\end{array}$ \\
\hline
\end{tabular}

HPLC Chromatogram of Compound ( \pm ) 3d (Manual mixing)
Sample Info

N-Me-DBU-DMF-MALONO-R

CHIRALPAK IA-3; 250 MM

IPA/HEXANE $: 15 / 85$

FLOW RATE: $1.0 \mathrm{~mL} / \mathrm{mi}$

$254 \mathrm{~nm}$

Additional Info : Peak(s) manually integrated

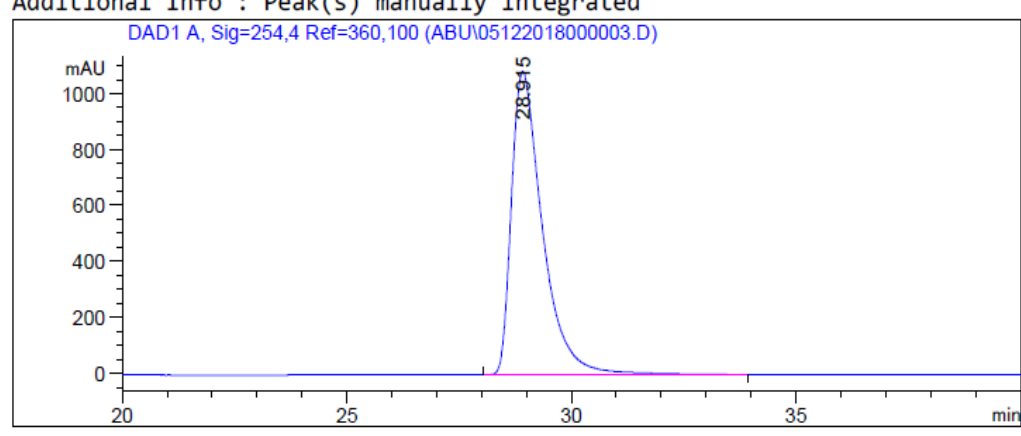

Area Percent Report

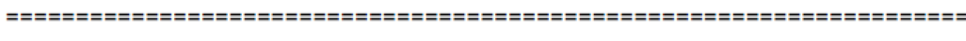

$\begin{array}{lll}\text { Sorted By } & : & \text { Signal } \\ \text { Multiplier } & : & 1.0000\end{array}$

$\begin{array}{lll}\text { Multiplier } & : & 1.0000 \\ \text { Dilution } & : & 1.0000\end{array}$

Use Multiplier \& Dilution Factor with ISTDs

Signal 1: DAD1 A, Sig $=254,4$ Ref $=360,100$

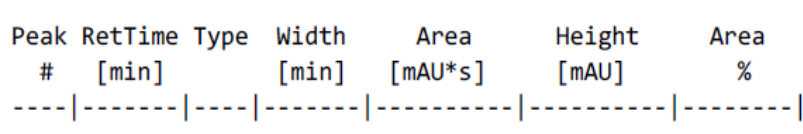

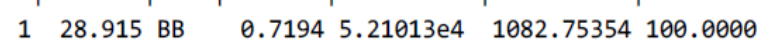

HPLC Chromatogram of Compound 3d 
Additional Info : Peak(s) manually integrated

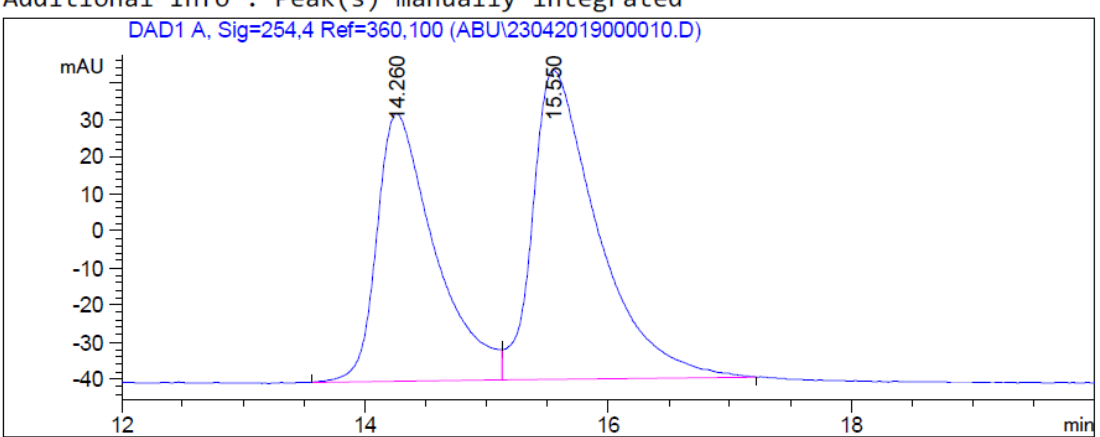

Sample Info

: N-BnZ-MALONO-S

CHIRALPAK IB-3; 250 MM

IPA/HEXANE : 15/85

FLOW RATE: $1 \mathrm{~mL} / \mathrm{min}$

$$
254 \mathrm{~nm}
$$

Additional Info : Peak(s) manually integrated

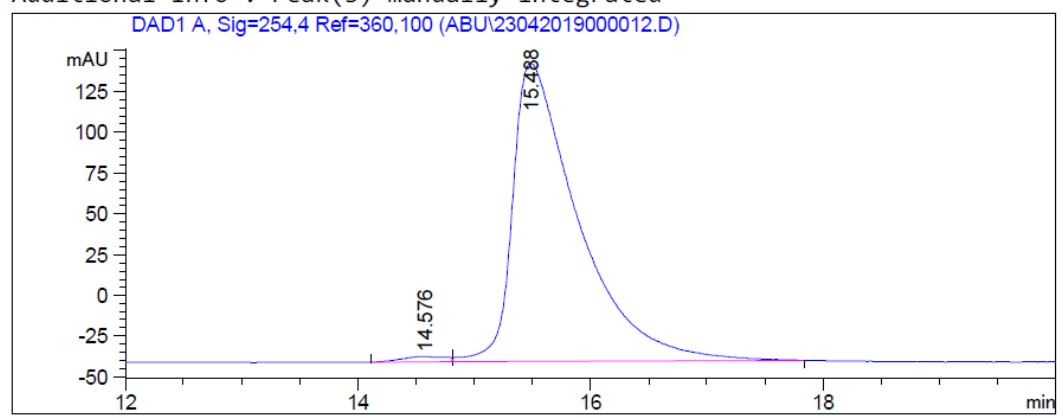

Area Percent Report

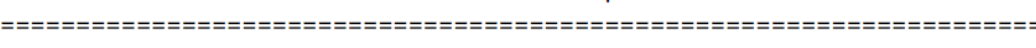

$\begin{array}{lll}\text { Sorted By } & : & \text { Signal } \\ \text { Multiplier } & : & 1.0000 \\ \text { Dilution } & : & 1.0000\end{array}$

Use Multiplier \& Dilution Factor with ISTDs

Signal 1: DAD1 A, Sig=254,4 Ref $=360,100$

\begin{tabular}{|c|c|c|c|c|c|}
\hline $\begin{array}{c}\text { Peak } \\
\#\end{array}$ & $\begin{array}{l}\text { RetTime Type } \\
\text { [min] }\end{array}$ & $\begin{array}{l}\text { Width } \\
\text { [min] }\end{array}$ & $\begin{array}{c}\text { Area } \\
{\left[\mathrm{mAU}^{*} \mathrm{~s}\right]}\end{array}$ & $\begin{array}{l}\text { Height } \\
{[\mathrm{mAU}]}\end{array}$ & $\begin{array}{c}\text { Area } \\
\%\end{array}$ \\
\hline 1 & 14. & a & 25 & 72 & 372 \\
\hline
\end{tabular}

HPLC Chromatogram of Compound ( \pm ) 3e (Manual mixing)

Area Percent Report

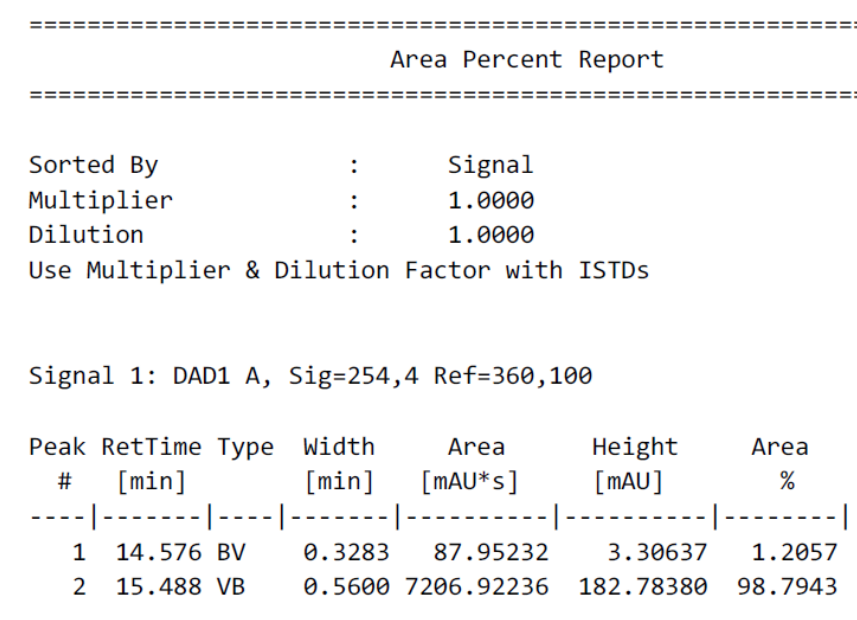

HPLC Chromatogram of Compound ent-3e 
CHIRALPAK IA- $3 ; 250$ MM

IPA/HEXANE : $10 / 90$

FLOW RATE: $1 \mathrm{~mL} / \mathrm{min}$

$254 \mathrm{~nm}$

Additional Info : Peak(s) manually integrated

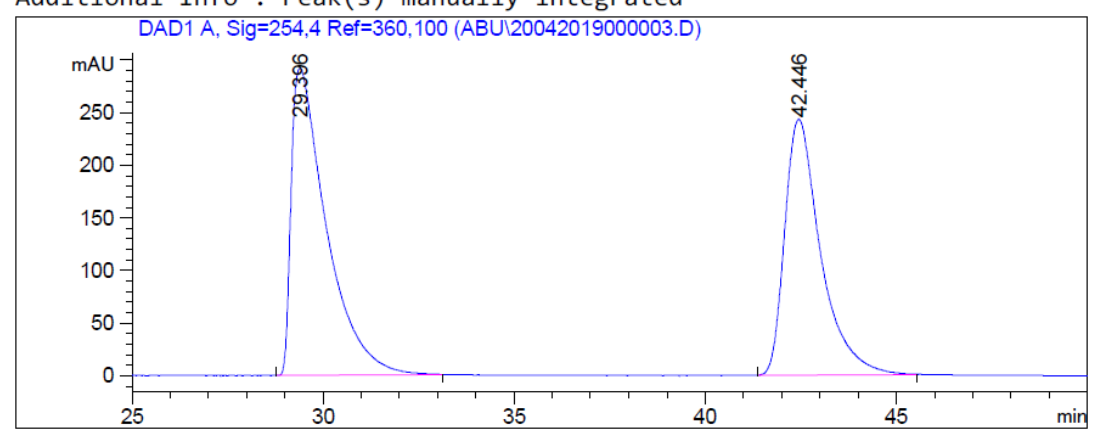

Area Percent Report

$\begin{array}{lll} & & \\ \text { Sorted By } & : & \text { Signal } \\ \text { Multiplier } & : & 1.0000 \\ \text { Dilution } & : & 1.0000\end{array}$

Use Multiplier \& Dilution Factor with ISTDS

Signal 1: DAD1 A, Sig=254,4 Ref $=360,100$

\begin{tabular}{|c|c|c|c|c|c|}
\hline $\begin{array}{l}\text { eak } \\
\#\end{array}$ & $\begin{array}{l}\text { RetTime Type } \\
\text { [min] }\end{array}$ & $\begin{array}{l}\text { Width } \\
\text { [min] }\end{array}$ & $\begin{array}{c}\text { Area } \\
{\left[\mathrm{mAU}^{*} \mathrm{~s}\right]}\end{array}$ & $\begin{array}{l}\text { Height } \\
{[\mathrm{mAU}]}\end{array}$ & $\begin{array}{c}\text { Area } \\
\%\end{array}$ \\
\hline & & & & & \\
\hline 1 & & & 86484 & 9 & 34 \\
\hline 2 & & & 995 e4 & 43.10358 & 46.0216 \\
\hline
\end{tabular}

HPLC Chromatogram of Compound ( \pm ) $3 f$ (Manual mixing)

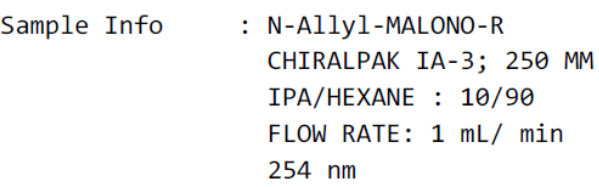

FLOW RATE: $1 \mathrm{~mL} / \mathrm{min}$ $254 \mathrm{~nm}$

Additional Info : Peak(s) manually integrated

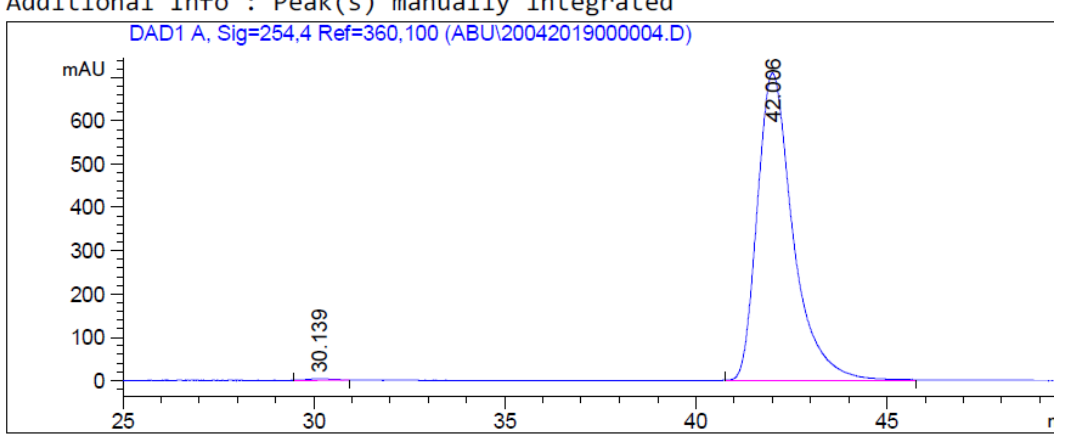

Area Percent Report

$\begin{array}{lll}\text { Sorted By } & : & \text { Signal } \\ \text { Multiplier } & : & 1.0000 \\ \text { Dilution } & : & 1.0000\end{array}$

Use Multiplier \& Dilution Factor with ISTD

Signal 1: DAD1 A, Sig=254,4 Ref=360,100

\begin{tabular}{|c|c|c|c|c|c|}
\hline $\begin{array}{l}\text { eak } \\
\#\end{array}$ & $\begin{array}{l}\text { RetTime Type } \\
\text { [min] }\end{array}$ & $\begin{array}{l}\text { Width } \\
\text { [min] }\end{array}$ & $\begin{array}{c}\text { Area } \\
{\left[\mathrm{mAU}^{*} \mathrm{~s}\right]}\end{array}$ & $\begin{array}{l}\text { Height } \\
{[\mathrm{mAU}]}\end{array}$ & $\begin{array}{c}\text { Area } \\
\%\end{array}$ \\
\hline 1 & $39 \mathrm{BB}$ & 12 & 154.90373 & 3.51479 & 0.3353 \\
\hline 2 & $42.006 \mathrm{BB}$ & 9665 & $4.60421 \mathrm{e} 4$ & 10.04126 & 647 \\
\hline
\end{tabular}

HPLC Chromatogram of Compound $\mathbf{3 f}$ 


$$
\begin{array}{ll}
\text { Sample Info } & \text { AS-N-Boc-malono-mix } \\
& \text { CHIRALPAK IA-3; } 250 \mathrm{MM} \\
& \text { IPA/HEXANE }: 10 / 90 \\
& \text { FLOW RATE: } 1.0 \mathrm{~mL} / \mathrm{min}
\end{array}
$$

Additional Info : Peak(s) manually integrated

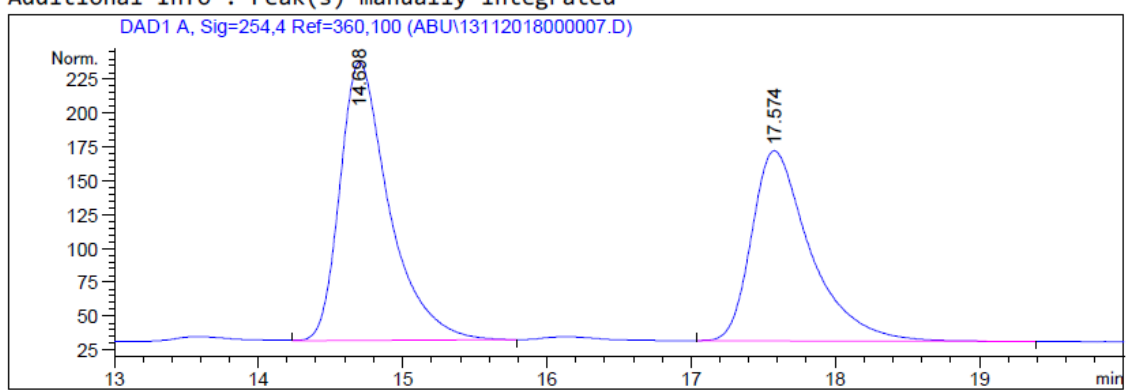

Area Percent Report

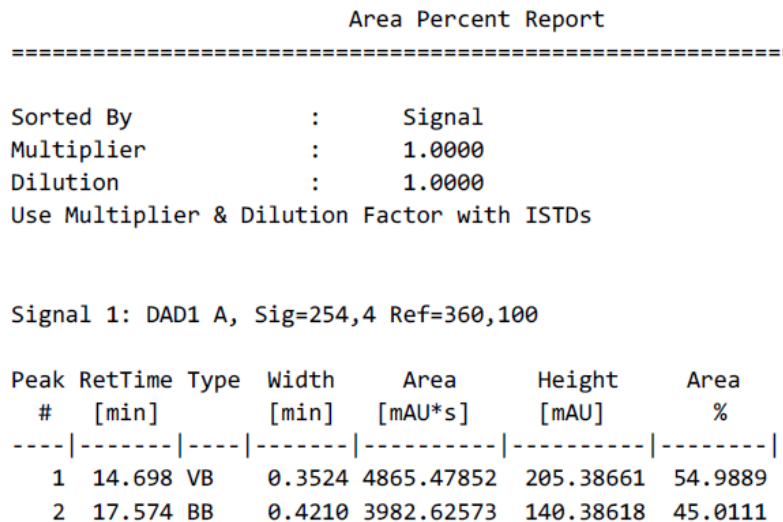

HPLC Chromatogram of Compound ( \pm ) 3g (Manual mixing)
Sample Info : AS-N-Boc-malono- $\mathrm{R}$
CHIRALPAK IA-3; 250 MM
IPA/HEXANE : $10 / 90$
FLOW RATE: $1.0 \mathrm{~mL}$ min

Additional Info : Peak(s) manually integrated

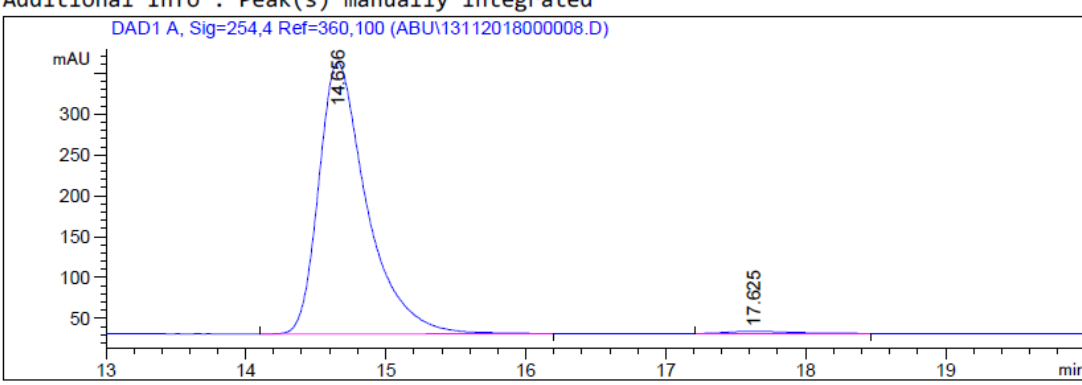

Area Percent Report

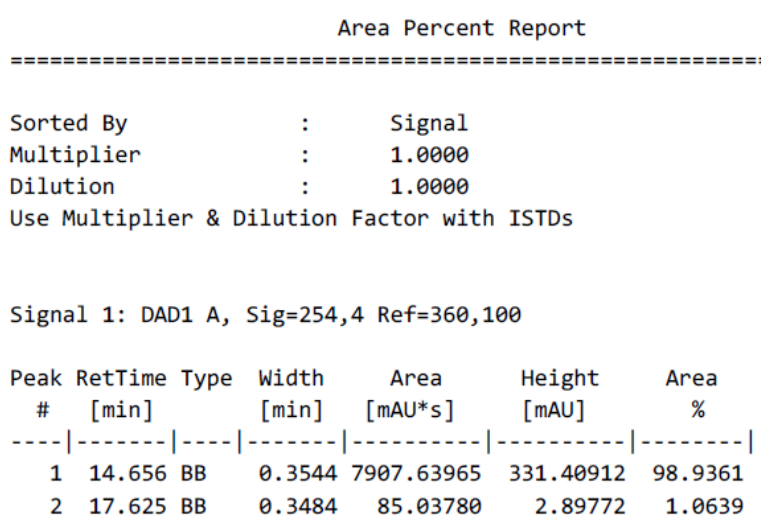

HPLC Chromatogram of Compound $\mathbf{3 g}$ 
Sample Info

NH FREE-MALONO-MIX

CHIRALPAK OJ-H; 250 MM

IPA/HEXANE : $15 / 85$

FLOW RATE: $1.0 \mathrm{~mL} / \mathrm{min}$

$254 \mathrm{~nm}$

Additional Info : Peak(s) manually integrated

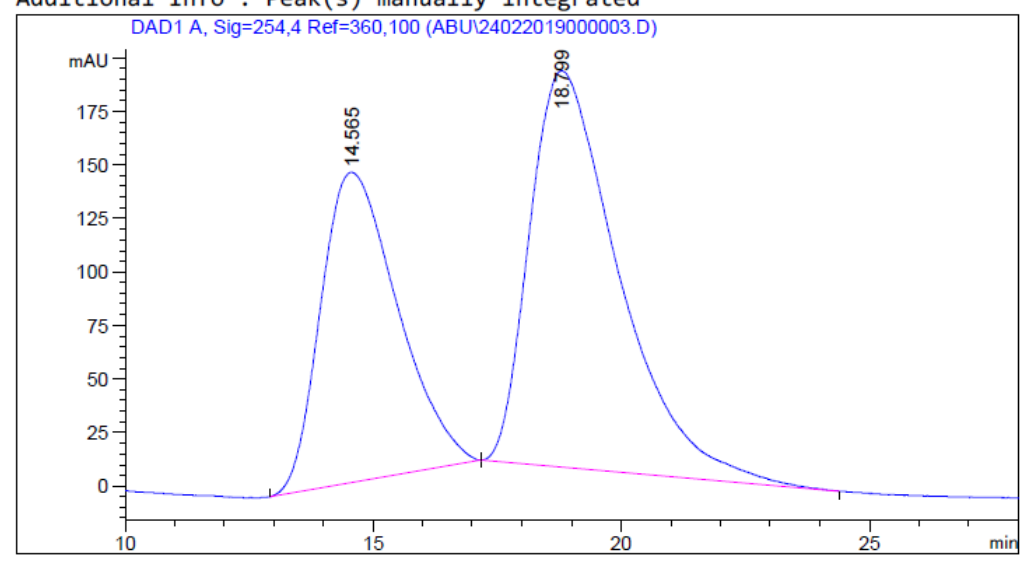

Area Percent Report

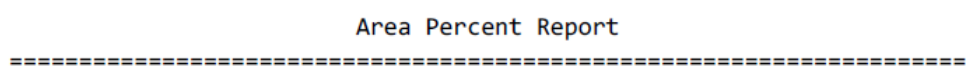

\section{Sorted By : Signal \\ : 1.0000 \\ 1.0000}

Dilution

Use Multiplier \& Dilution Factor with ISTDs

Signal 1: DAD1 A, Sig $=254,4$ Ref $=360,100$

\begin{tabular}{|c|c|c|c|c|c|}
\hline $\begin{array}{c}\text { Peak } \\
\#\end{array}$ & $\begin{array}{l}\text { RetTime Type } \\
\text { [min] }\end{array}$ & $\begin{array}{l}\text { Width } \\
\text { [min] }\end{array}$ & $\begin{array}{c}\text { Area } \\
{\left[\mathrm{mAU}^{*} \mathrm{~s}\right]}\end{array}$ & $\begin{array}{l}\text { Height } \\
{[\mathrm{mAU}]}\end{array}$ & $\begin{array}{c}\text { Area } \\
\%\end{array}$ \\
\hline & 1 & & 1.558 & 145. & 697 \\
\hline 2 & 18.799 ВB & 4771 & $2.32164 \mathrm{e} 4$ & 185.12378 & 59.8303 \\
\hline
\end{tabular}

HPLC Chromatogram of Compound ( \pm ) $3 \mathbf{h}$ (Manual mixing)
Sample Info : NH FREE-MALONO-S

CHIRALPAK OJ-H; 250 MM

IPA/HEXANE : $15 / 85$

FLOW RATE: $1.0 \mathrm{~mL} / \mathrm{min}$

$254 \mathrm{~nm}$

Additional Info : Peak(s) manually integrated

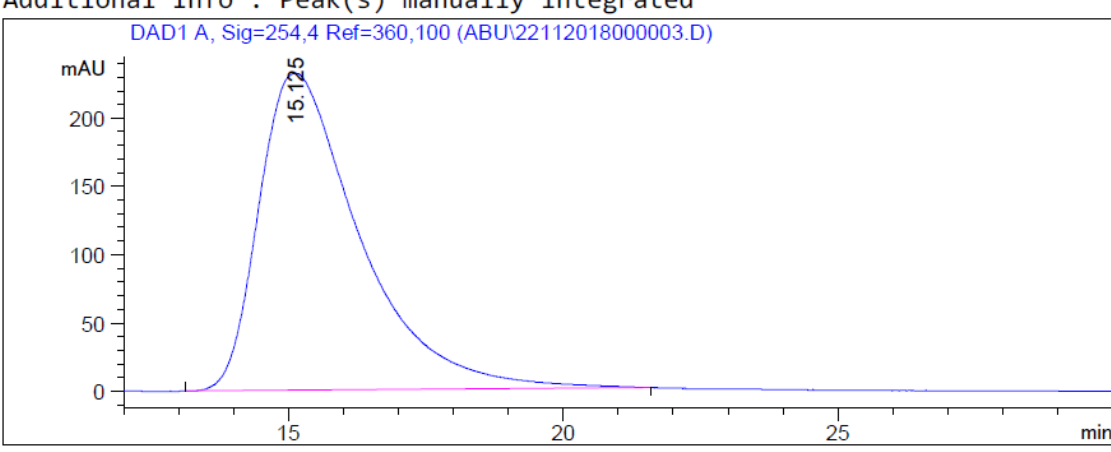

Area Percent Report

$\begin{array}{lll}\text { Sorted By } & : & \text { Signal } \\ \text { Multiplier } & : & 1.0000 \\ \text { Dilution } & : & 1.0000\end{array}$

Use Multiplier \& Dilution Factor with ISTDs

Signal 1: DAD1 A, Sig $=254,4$ Ref $=360,100$

\begin{tabular}{|c|c|c|c|c|}
\hline $\begin{array}{l}\text { Peak RetTime Type } \\
\# \quad[\text { min }]\end{array}$ & $\begin{array}{l}\text { Width } \\
\text { [min] }\end{array}$ & $\begin{array}{c}\text { Area } \\
{\left[\mathrm{mAU}^{*} \mathrm{~s}\right]}\end{array}$ & $\begin{array}{l}\text { Height } \\
{[\mathrm{mAU}]}\end{array}$ & $\begin{array}{c}\text { Area } \\
\%\end{array}$ \\
\hline
\end{tabular}

15.125 BB $1.51132 .9766904 \quad 232.24408100 .0000$

HPLC Chromatogram of Compound ent $\mathbf{- 3 h}$ 
IPA/HEXANE : $25 / 75$

FLOW RATE: $1 \mathrm{~mL} / \mathrm{min}$

$254 \mathrm{~nm}$

Additional Info : Peak(s) manually integrated

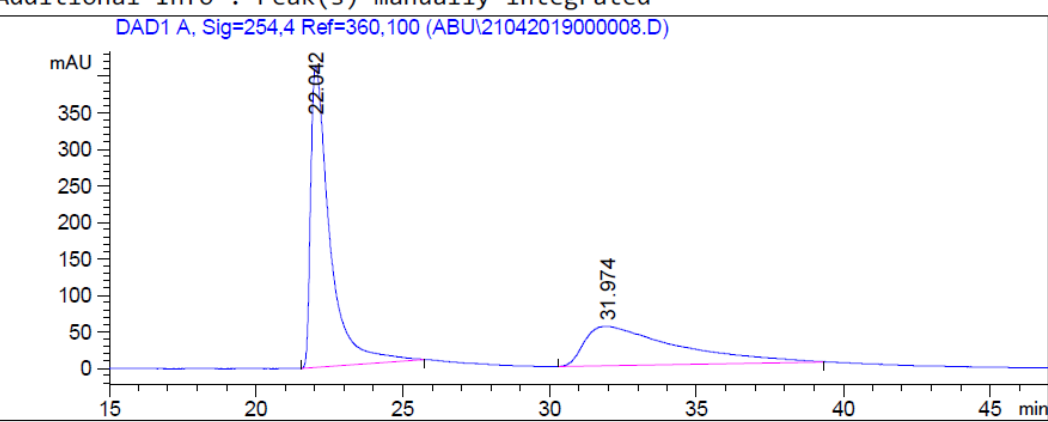

Area Percent Report

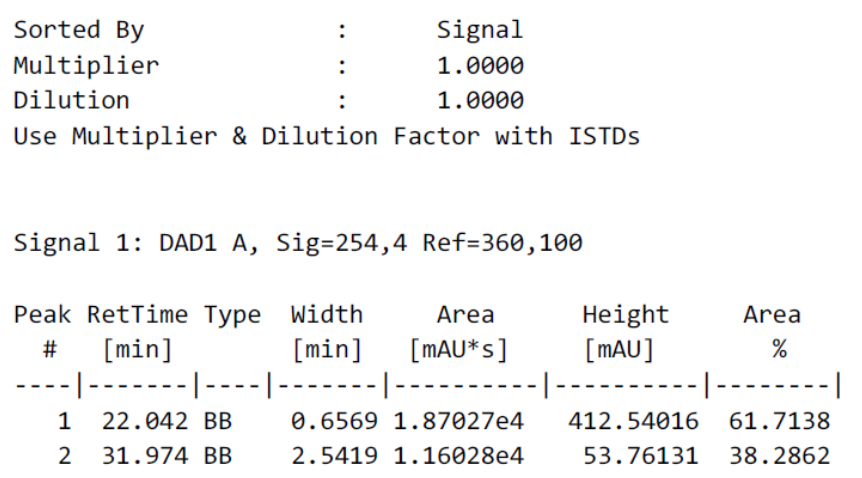

HPLC Chromatogram of Compound ( \pm ) 3i (Manual mixing)
Sample Info

: N-Me-5-OMe-MALONO-R

CHIRALPAK IA-3; 250 MM

IPA/HEXANE : $25 / 75$

FLOW RATE: $1 \mathrm{~mL} / \mathrm{min}$

$254 \mathrm{~nm}$

Additional Info : Peak(s) manually integrated

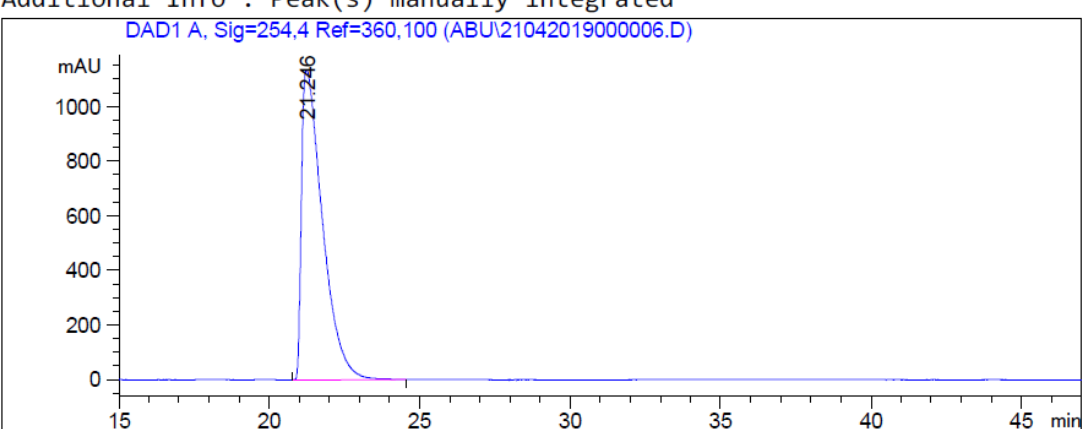

Area Percent Report

$\begin{array}{lll}\text { Sorted By } & : & \text { Signal } \\ \text { Multiplier } & : & 1.0000 \\ \text { Dilution } & : & 1.0000\end{array}$

Dilution : 1.0000

Use Multiplier \& Dilution Factor with ISTDs

Signal 1: DAD1 A, Sig=254,4 Ref=360,100

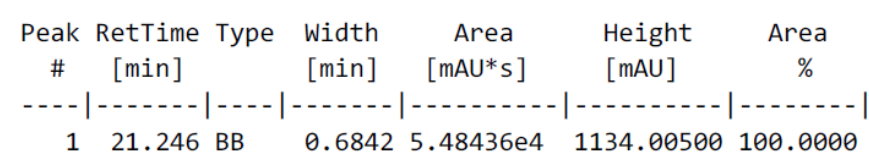

HPLC Chromatogram of Compound 3i 
Sample Info

N-Me-5-Me-MALONO-MIX

CHIRALPAK IA-3; 250 MM

IPA/HEXANE $: 10 / 90$

FLOW RATE: $1.0 \mathrm{~mL} / \mathrm{min}$

$254 \mathrm{~nm}$

Additional Info : Peak(s) manually integrated

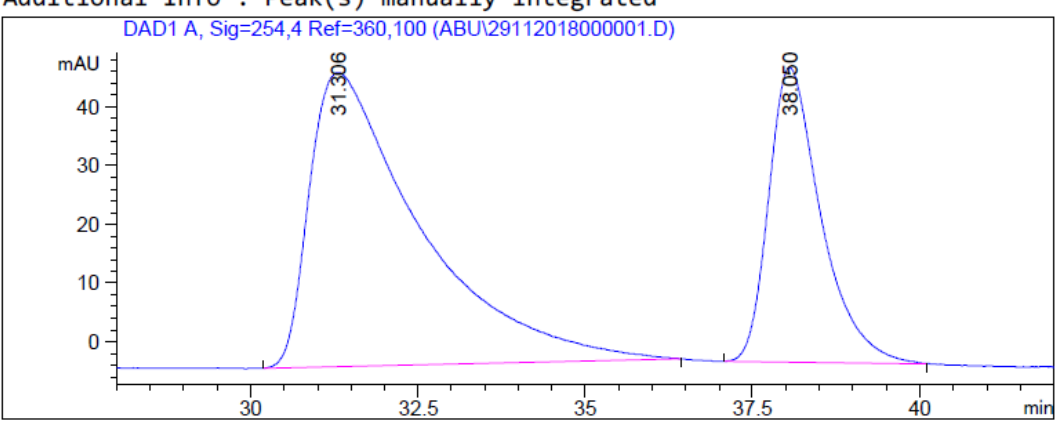

Area Percent Report

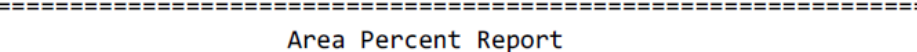

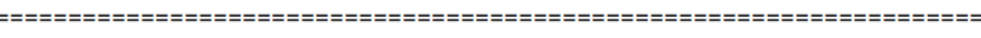

$\begin{array}{lll}\text { Sorted By } & : & \text { Signal } \\ \text { Multiplier } & : & 1.0000 \\ \text { Dilution } & : & 1.0000\end{array}$

$: \quad 1.0000$

Use Multiplier \& Dilution Factor with ISTDs

Signal 1: DAD1 A, Sig $=254,4$ Ref $=360,100$

\begin{tabular}{|c|c|c|c|c|c|}
\hline $\begin{array}{c}\text { Peak } \\
\#\end{array}$ & $\begin{array}{l}\text { RetTime Type } \\
\text { [min] }\end{array}$ & $\begin{array}{l}\text { Width } \\
\text { [min] }\end{array}$ & $\begin{array}{c}\text { Area } \\
{[\mathrm{mAU} * \mathrm{~s}]}\end{array}$ & $\begin{array}{l}\text { Height } \\
{[\mathrm{mAU}]}\end{array}$ & $\begin{array}{c}\text { Area } \\
\%\end{array}$ \\
\hline & & & & & \\
\hline 1 & 06 & 1.3808 & 5773.54980 & 50.01345 & 837 \\
\hline 2 & $38.050 \mathrm{BB}$ & 0.7852 & 2718.99951 & 50.09531 & 32.0163 \\
\hline
\end{tabular}

HPLC Chromatogram of Compound ( \pm ) 3j (Manual mixing)
Sample Info : N-Me-5-Me-MALONO-S

CHIRALPAK IA- $3 ; 250$ MM

IPA/HEXANE $: 10 / 90$

FLOW RATE: $1.0 \mathrm{~mL} / \mathrm{min}$

$254 \mathrm{~nm}$

Additional Info : Peak(s) manually integrated

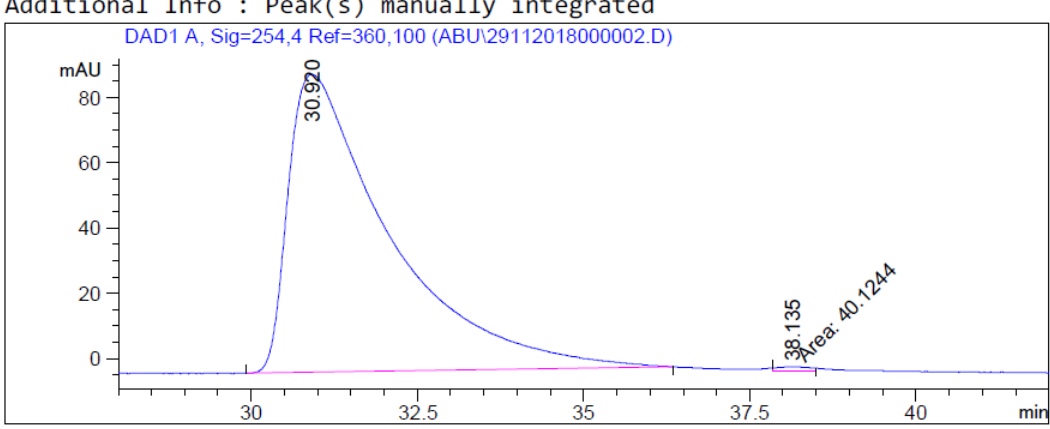

Area Percent Report

Sorted By : Signal

Multiplier $\quad: \quad 1.0000$

Dilution : 1.0000

Use Multiplier \& Dilution Factor with ISTDs

Signal 1: DAD1 A, Sig $=254,4$ Ref $=360,100$

\begin{tabular}{|c|c|c|c|c|c|}
\hline $\begin{array}{c}\text { Peak } \\
\#\end{array}$ & $\begin{array}{l}\text { RetTime Type } \\
\text { [min] }\end{array}$ & $\begin{array}{l}\text { Width } \\
{[\mathrm{min}]}\end{array}$ & $\begin{array}{c}\text { Area } \\
{[\mathrm{mAU} * \mathrm{~s}]}\end{array}$ & $\begin{array}{l}\text { Height } \\
{[\mathrm{mAU}]}\end{array}$ & $\begin{array}{c}\text { Area } \\
\%\end{array}$ \\
\hline & 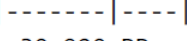 & & (1) & 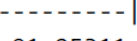 & \\
\hline 1 & $30.920 \mathrm{BB}$ & 1.4007 & 9673.13086 & 91.25 & 99.5869 \\
\hline 2 & $38.135 \mathrm{MM}$ & 0.5570 & 40.12443 & 1.20067 & 0.4131 \\
\hline
\end{tabular}

\section{HPLC Chromatogram of Compound ent-3j}


IPA/HEXANE : $15 / 85$

FLOW RATE: $1 \mathrm{~mL} / \mathrm{min}$

$$
254 \mathrm{~nm}
$$

Additional Info : Peak(s) manually integrated

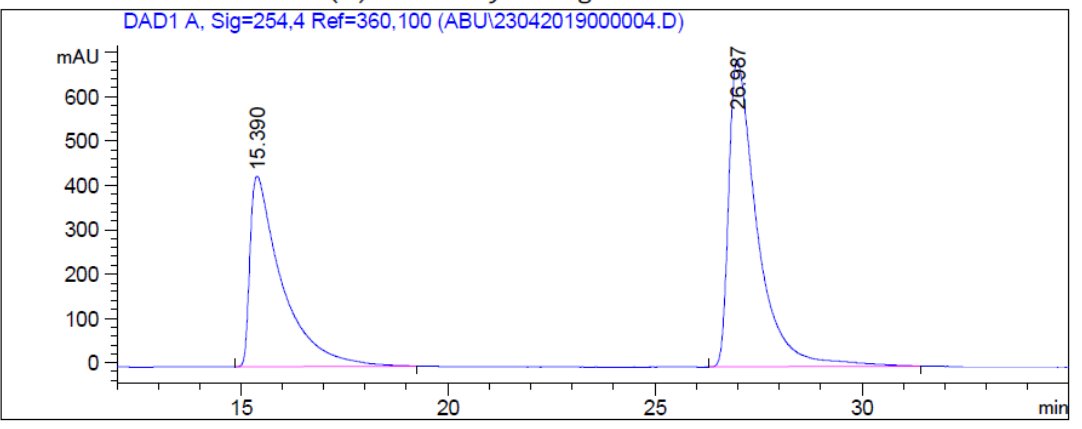

Area Percent Report

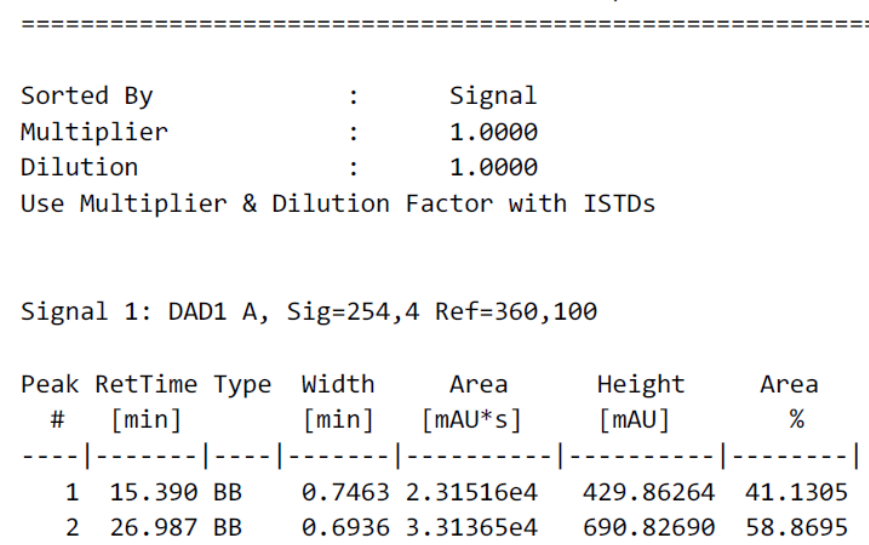

HPLC Chromatogram of Compound ( \pm ) 3k (Manual mixing)
Sample Info

$$
\begin{aligned}
& \text { N-Me-5,7-Di-Me-MALONO-R } \\
& \text { CHIRALPAK IA-3; } 250 \text { MM } \\
& \text { IPA/HEXANE : 15/85 } \\
& \text { FLOW RATE: } 1 \mathrm{~mL} / \mathrm{min}
\end{aligned}
$$$$
254 \mathrm{~nm}
$$

Additional Info : Peak(s) manually integrated

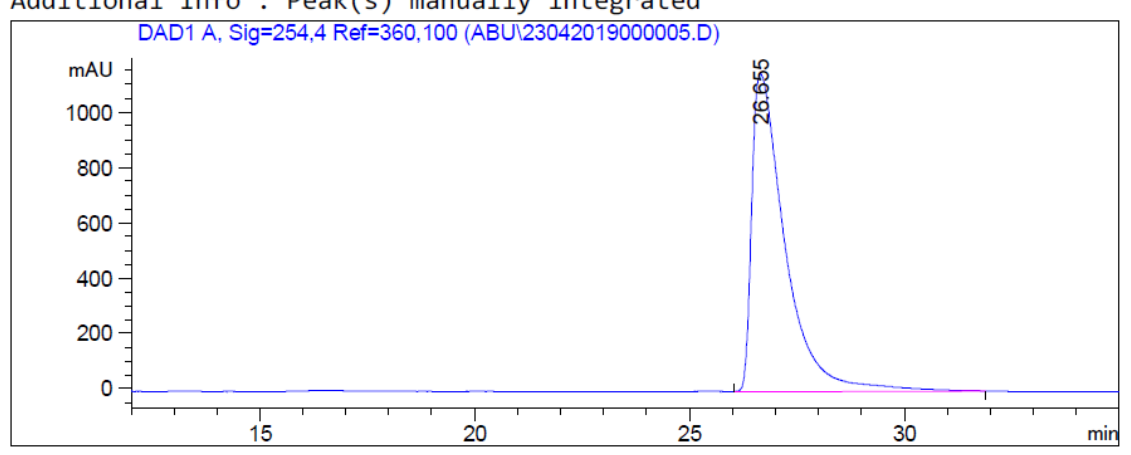

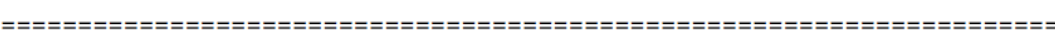

Area Percent Report

$\begin{array}{lll} & & \\ \text { Sorted By } & : & \text { Signal } \\ \text { Multiplier } & : & 1.0000 \\ \text { Dilution } & : & 1.0000\end{array}$

Dilution

Use Multiplier \& Dilution Factor with ISTDS

Signal 1: DAD1 A, Sig $=254,4$ Ref $=360,100$

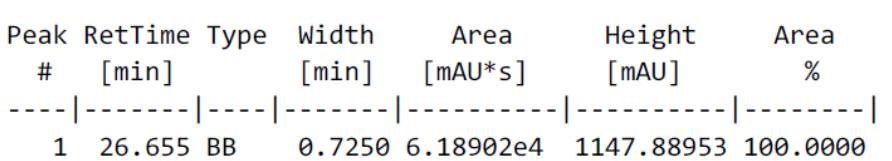

126.655 вВ $0.7250 \quad 6.18902 \mathrm{e} 4 \quad 1147.88953 \quad 100.0000$

\section{HPLC Chromatogram of Compound 3k}


Sample Info

N-Me-5-Cl-malono-mix

CHIRALPAK IB-3; 250 MM

IPA/HEXANE : $15 / 85$

FLOW RATE: $1.0 \mathrm{~mL} / \mathrm{min}$

Additional Info : Peak(s) manually integrated

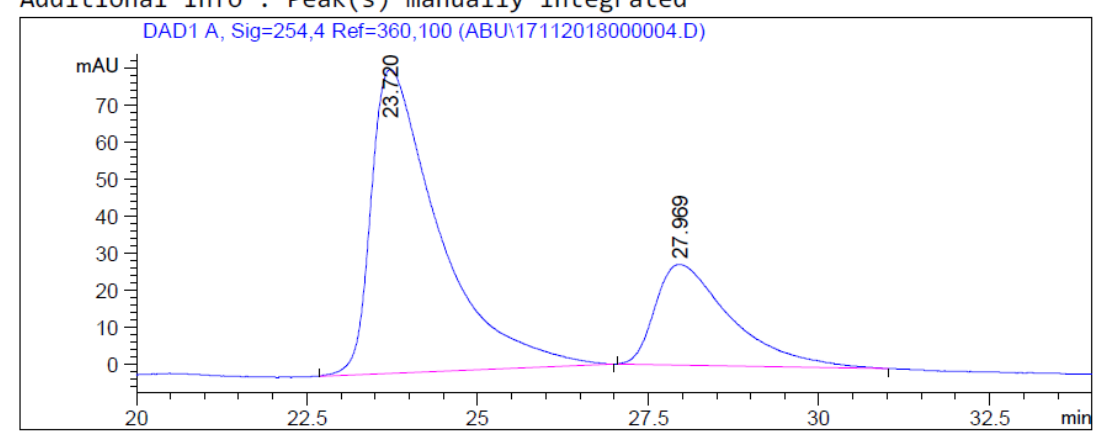

Area Percent Report

\section{Sorted By \\ Multiplier \\ Dilution \\ Signal \\ 1.0000}

Use Multiplier \& Dilution Factor with ISTDs

Signal 1: DAD1 A, Sig=254,4 Ref $=360,100$

\begin{tabular}{|c|c|c|c|c|c|c|}
\hline $\begin{array}{l}\text { eak } \\
\#\end{array}$ & $\begin{array}{c}\text { RetTime } \\
\text { [min] }\end{array}$ & & $\begin{array}{l}\text { Width } \\
\text { [min] }\end{array}$ & $\begin{array}{c}\text { Area } \\
{\left[\mathrm{mAU}^{*} \mathrm{~s}\right]}\end{array}$ & $\begin{array}{l}\text { Height } \\
{[\mathrm{mAU}]}\end{array}$ & $\begin{array}{c}\text { Area } \\
\%\end{array}$ \\
\hline & & & & & & \\
\hline 2 & & & & 82.37256 & $\begin{array}{l}81.99905 \\
27.12206\end{array}$ & 26.8150 \\
\hline
\end{tabular}

HPLC Chromatogram of Compound ( \pm ) 3m (Manual mixing) $\begin{array}{ll}\text { Sample Info } & \text { N-Me-5-Cl-malono-S } \\ & \text { CHIRALPAK IB- } 3 ; 250 \mathrm{MM} \\ & \text { IPA/HEXANE }: 15 / 85 \\ & \text { FLOW RATE: } 1.0 \mathrm{~mL} / \mathrm{min}\end{array}$

Additional Info : Peak(s) manually integrated

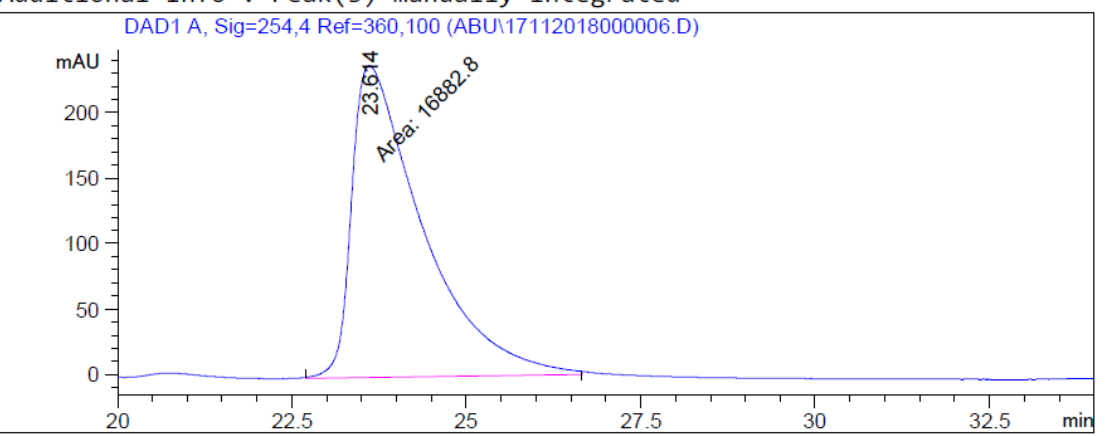

Area Percent Report

$\begin{array}{lll}\text { Sorted By } & : & \text { Signal } \\ \text { Multiplier } & : & 1.0000 \\ \text { Dilution } & : & 1.0000\end{array}$

Use Multiplier \& Dilution Factor with ISTDS

Signal 1: DAD1 A, Sig $=254,4$ Ref $=360,100$

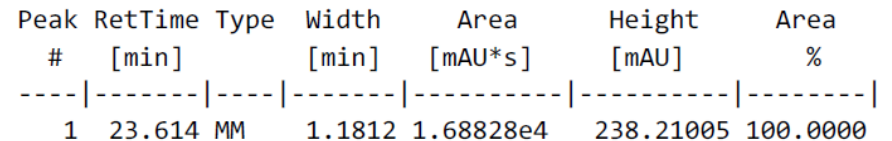

\section{HPLC Chromatogram of Compound ent-3m}


CHIRALPAK IA-3; 250 MM

IPA/HEXANE : 10/90

FLOW RATE: $1 \mathrm{~mL} / \mathrm{min}$

$254 \mathrm{~nm}$

Additional Info : Peak(s) manually integrated

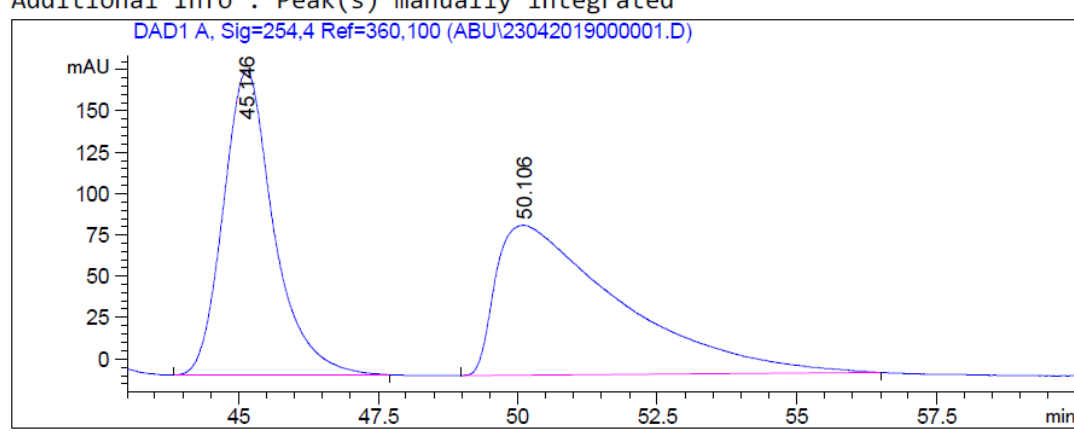

Area Percent Report

\begin{tabular}{|c|c|c|c|c|}
\hline Sorted By & $:$ & Signal & & \\
\hline Multiplier & : & 1.0000 & & \\
\hline Dilution & : & 1.0000 & & \\
\hline \multicolumn{5}{|c|}{ Use Multiplier \& Dilution Factor with ISTDs } \\
\hline \multicolumn{5}{|c|}{ Signal 1: DAD1 A, Sig $=254,4$ Ref $=360,100$} \\
\hline $\begin{array}{l}\text { Peak RetTime Type } \\
\# \quad[\text { min }]\end{array}$ & $\begin{array}{l}\text { Width } \\
\text { [min] }\end{array}$ & $\begin{array}{c}\text { Area } \\
{[\mathrm{mAU} * \mathrm{~s}]}\end{array}$ & $\begin{array}{l}\text { Height } \\
{[\mathrm{mAU}]}\end{array}$ & $\begin{array}{c}\text { Area } \\
\%\end{array}$ \\
\hline 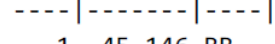 & -1 & 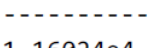 & -10-1 & \\
\hline $145.146 \mathrm{BB}$ & 0.9165 & $1.16024 \mathrm{e} 4$ & 184.29576 & 45.2073 \\
\hline 250.106 BB & 1.8195 & $1.40625 \mathrm{e} 4$ & 90.96800 & 54.7927 \\
\hline
\end{tabular}

HPLC Chromatogram of Compound ( \pm ) 3n (Manual mixing)
CHIRALPAK IA-3; 250 MM

IPA/HEXANE : $10 / 90$

FLOW RATE: $1 \mathrm{~mL} / \mathrm{min}$

$254 \mathrm{~nm}$

Additional Info : Peak(s) manually integrated

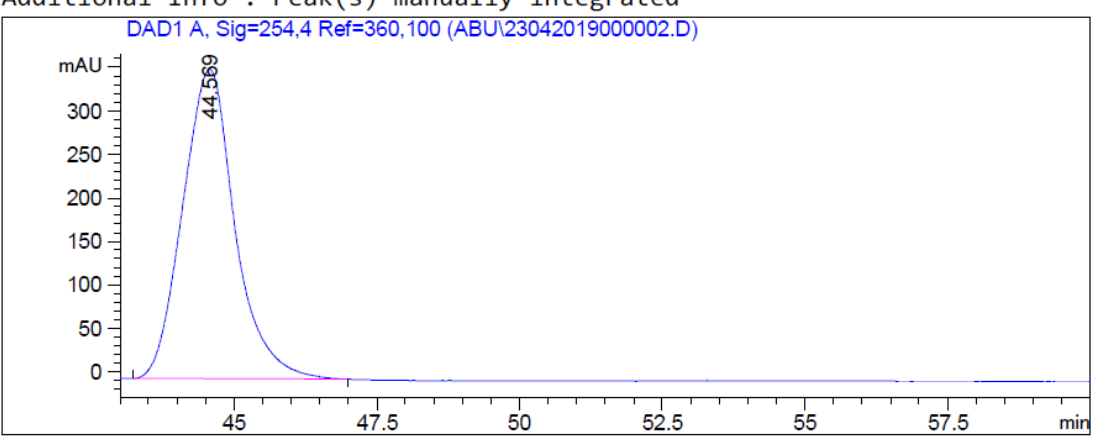

Area Percent Report

$\begin{array}{lll}\text { Sorted By } & : & \text { Signal } \\ \text { Multiplier } & : & 1.0000 \\ \text { Dilution } & : & 1.0000\end{array}$

Use Multiplier \& Dilution Factor with ISTDs

Signal 1: DAD1 A, Sig $=254,4$ Ref $=360,100$

\begin{tabular}{ccccc}
$\begin{array}{c}\text { Peak RetTime Type Width } \\
\text { \# [min] }\end{array}$ [min] & $\begin{array}{c}\text { Area } \\
{[\mathrm{mAU} \text { s }]}\end{array}$ & $\begin{array}{c}\text { Height } \\
{[\mathrm{mAU}]}\end{array}$ & $\begin{array}{c}\text { Area } \\
\%\end{array}$ \\
\hdashline $144.569 \mathrm{BB}$ & 0.9379 & $2.26950 \mathrm{e} 4$ & 355.93842 & 100.0000
\end{tabular}

HPLC Chromatogram of Compound 3n 
Sample Info

N-Me-5-I-MALONO-MIX

CHIRALPAK IA-3; 250 MM

IPA/HEXANE : $10 / 90$

FLOW RATE: $1 \mathrm{~mL} / \mathrm{min}$

$254 \mathrm{~nm}$

Additional Info : Peak(s) manually integrated

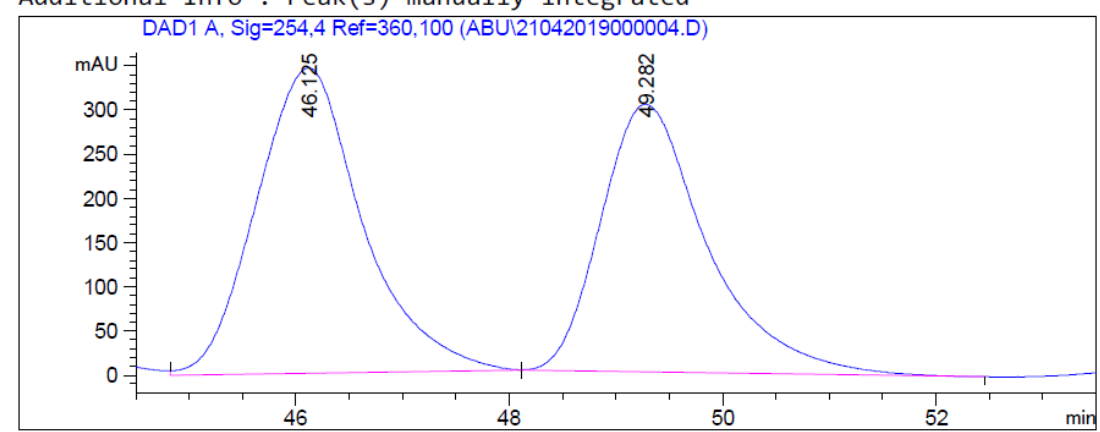

46

Area Percent Report

\begin{tabular}{|c|c|c|c|c|}
\hline Sorted By & $:$ & Signal & & \\
\hline Multiplier & : & 1.0000 & & \\
\hline Dilution & : & 1.0000 & & \\
\hline \multicolumn{5}{|c|}{ Use Multiplier \& Dilution Factor with ISTDs } \\
\hline \multicolumn{5}{|c|}{ Signal 1: DAD1 A, Sig=254,4 Ref $=360,100$} \\
\hline $\begin{array}{l}\text { Peak RetTime Type } \\
\# \quad[\min ]\end{array}$ & $\begin{array}{l}\text { Width } \\
{[\text { min] }}\end{array}$ & $\begin{array}{c}\text { Area } \\
{\left[\mathrm{mAU}^{*} \mathrm{~s}\right]}\end{array}$ & $\begin{array}{l}\text { Height } \\
{[\mathrm{mAU}]}\end{array}$ & $\begin{array}{c}\text { Area } \\
\%\end{array}$ \\
\hline $\begin{array}{ll}-1 & --n\end{array}$ & $-\cdots$ & - - - - & | & | \\
\hline $146.125 \mathrm{VB}$ & 1.0076 & $2.37688 \mathrm{e} 4$ & 345.08890 & 53.1518 \\
\hline $249.282 \mathrm{BB}$ & 1.0158 & $2.09499 \mathrm{e} 4$ & 302.56998 & 46.8482 \\
\hline
\end{tabular}

HPLC Chromatogram of Compound ( \pm ) 3o (Manual mixing)
Sample Info : N-Me-5-I-MALONO-R

CHIRALPAK IA-3; 250 MM

IPA/HEXANE : 10/90

FLOW RATE: $1 \mathrm{~mL} / \mathrm{min}$

$254 \mathrm{~nm}$

Additional Info : Peak(s) manually integrated

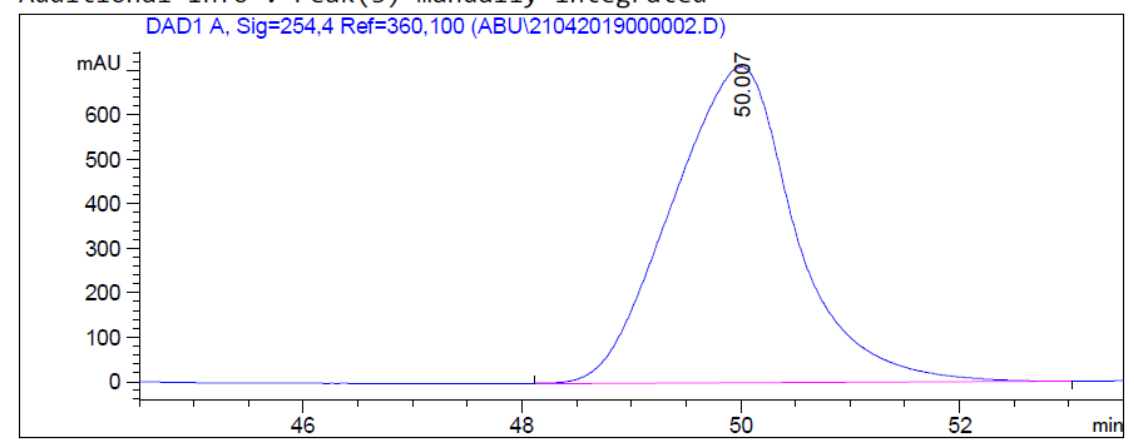

Area Percent Report

$\begin{array}{lll}\text { Sorted By } & : & \text { Signal } \\ \text { Multiplier } & : & 1.0000 \\ \text { Dilution } & : & 1.0000\end{array}$

Use Multiplier \& Dilution Factor with ISTDS

Signal 1: DAD1 A, Sig $=254,4$ Ref $=360,100$

\begin{tabular}{ccccc}
$\begin{array}{c}\text { Peak RetTime Type Width } \\
\text { \# [min] }\end{array}$ [min] $\begin{array}{c}\text { Area } \\
{[\mathrm{mAU} \text { *s }]}\end{array}$ & $\begin{array}{l}\text { Height } \\
{[\mathrm{mAU}]}\end{array}$ & $\begin{array}{c}\text { Area } \\
\%\end{array}$ \\
\hdashline 150.007 BB & 1.0551 & $5.58075 \mathrm{e} 4$ & 709.97882 & 100.0000
\end{tabular}

HPLC Chromatogram of Compound 30 
Sample Info

N-Me-7-F-MALONO-MIX

CHIRALPAK IA-3; 250 MM

IPA/HEXANE : $10 / 90$

FLOW RATE: $1.0 \mathrm{~mL} / \mathrm{min}$

$254 \mathrm{~nm}$

Additional Info : Peak(s) manually integrated

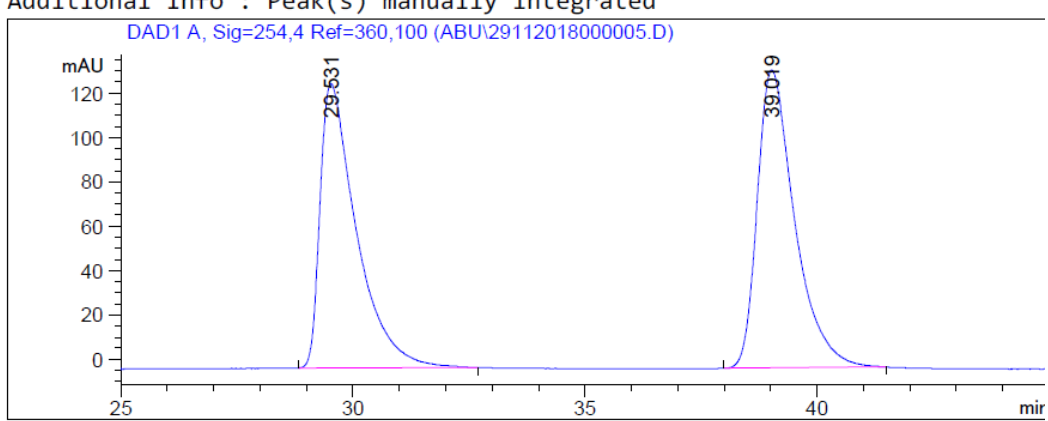

Area Percent Report

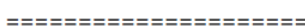

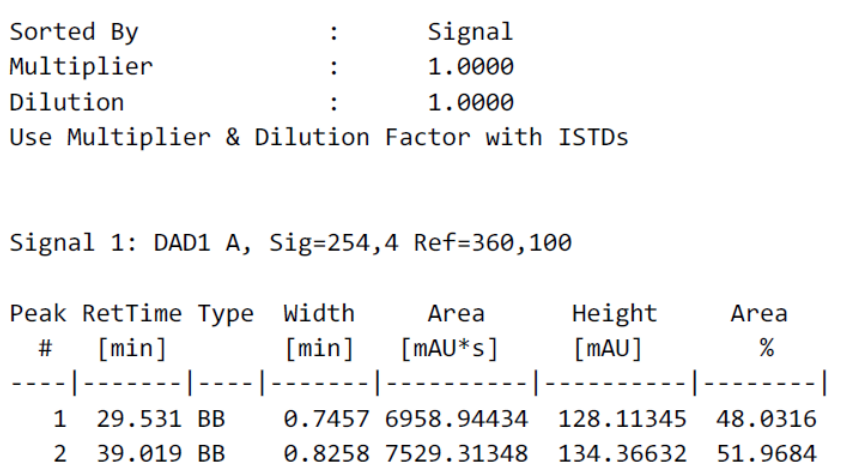

HPLC Chromatogram of Compound ( \pm ) 3p (Manual mixing)
Sample Info : N-Me-7-F-MALONO-R

CHIRALPAK IA-3; $250 \mathrm{MM}$

IPA/HEXANE $: 10 / 90$

FLOW RATE: $1.0 \mathrm{~mL} / \mathrm{min}$$$
254 \mathrm{~nm}
$$

Additional Info : Peak(s) manually integrated

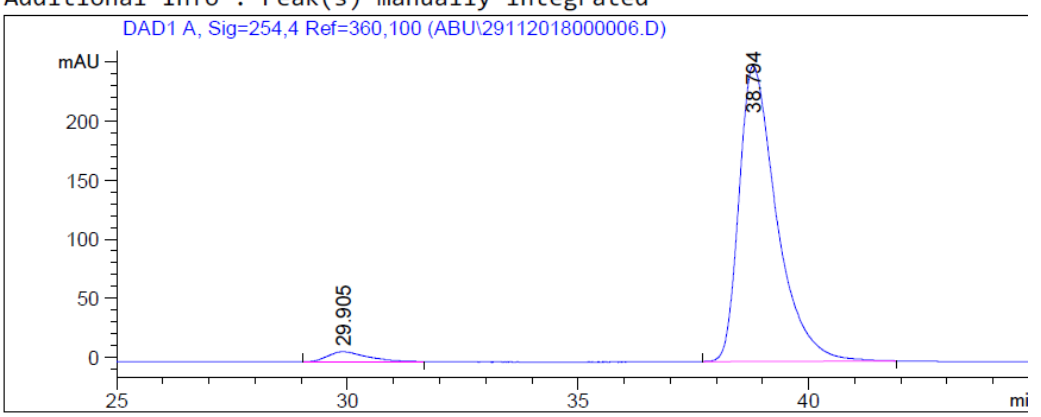

Area Percent Report

$\begin{array}{lll}\text { Sorted By } & : & \text { Signal } \\ \text { Multiplier } & : & 1.0000\end{array}$

Dilution

1.0000

Use Multiplier \& Dilution Factor with ISTDs

Signal 1: DAD1 A, Sig $=254,4$ Ref $=360,100$

\begin{tabular}{|c|c|c|c|c|c|}
\hline eak & $\begin{array}{l}\text { RetTime Type } \\
\text { [min] }\end{array}$ & $\begin{array}{l}\text { Width } \\
{[\text { min] }}\end{array}$ & $\begin{array}{c}\text { Area } \\
{[\mathrm{mAU} * \mathrm{~s}]}\end{array}$ & $\begin{array}{l}\text { Height } \\
{[\mathrm{mAU}]}\end{array}$ & $\begin{array}{c}\text { Area } \\
\%\end{array}$ \\
\hline & & & & & \\
\hline & & & & & \\
\hline
\end{tabular}

\section{HPLC Chromatogram of Compound 3p}


Sample Info

AS-7-CI-MALONO-MIX

CHIRALPAK IA-3; 250 MM

IPA/HEXANE : $15 / 85$

254 NM

FLOW RATE: $1.0 \mathrm{~mL} / \mathrm{min}$

Additional Info : Peak(s) manually integrated

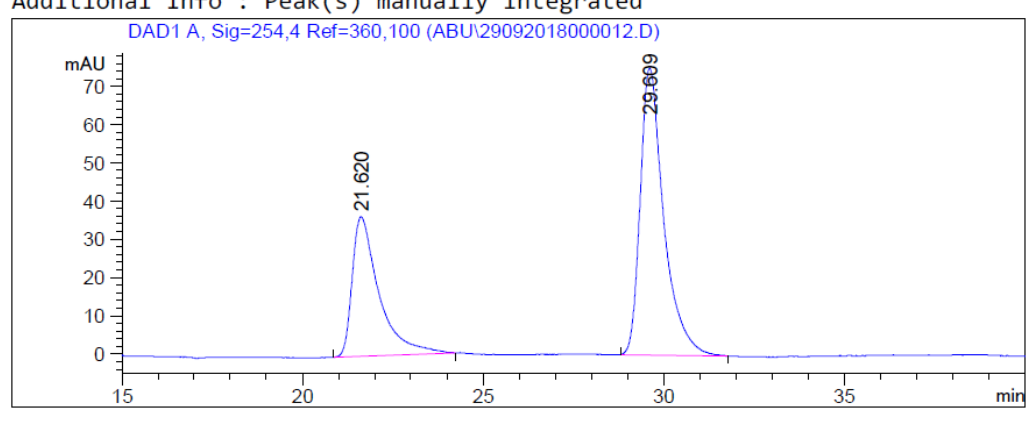

Area Percent Report

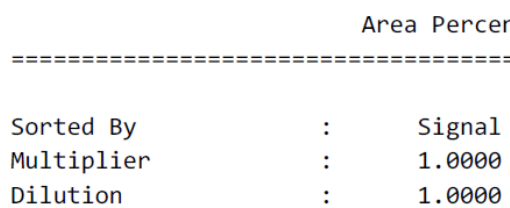

Use Multiplier \& Dilution Factor with ISTDs

Signal 1: DAD1 A, Sig=254,4 Ref $=360,100$

\begin{tabular}{|c|c|c|c|c|c|}
\hline $\begin{array}{l}\text { eak } \\
\#\end{array}$ & $\begin{array}{l}\text { RetTime Type } \\
\text { [min] }\end{array}$ & $\begin{array}{l}\text { Width } \\
\text { [min] }\end{array}$ & $\begin{array}{c}\text { Area } \\
{[\mathrm{mAU} * \mathrm{~s}]}\end{array}$ & $\begin{array}{l}\text { Height } \\
{[\mathrm{mAU}]}\end{array}$ & $\begin{array}{c}\text { Area } \\
\%\end{array}$ \\
\hline . & $\mathrm{BB}$ & 0.7196 & 1881.67615 & 36.49340 & 35.6289 \\
\hline 2 & $29.609 \mathrm{BB}$ & 0.6763 & 3399.63940 & 75.07229 & 64.3711 \\
\hline
\end{tabular}

HPLC Chromatogram of Compound ( \pm ) 3q (Manual mixing)
Sample Info : AS-7-Cl-MALONO-R

CHIRALPAK IA-3; 250 MM

IPA/HEXANE : $15 / 85$

254 NM

FLOW RATE: $1.0 \mathrm{~mL} / \mathrm{min}$

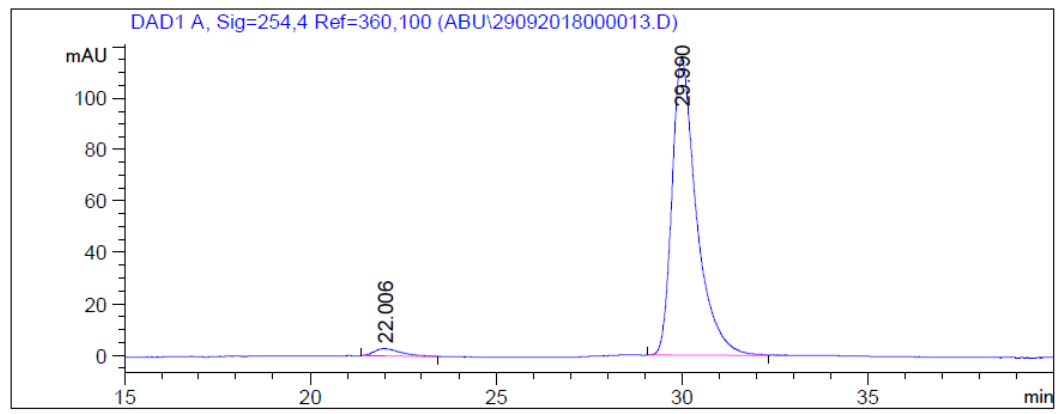

Area Percent Report

\section{Sorted By : Signal}

Multiplier $\quad: \quad 1.0000$

Dilution : 1.0000

Use Multiplier \& Dilution Factor with ISTDs

Signal 1: DAD1 A, Sig $=254,4$ Ref $=360,100$

\begin{tabular}{|c|c|c|c|c|c|}
\hline $\begin{array}{l}\text { eak } \\
\text { \# }\end{array}$ & $\begin{array}{l}\text { RetTime Type } \\
\text { [min] }\end{array}$ & $\begin{array}{l}\text { Width } \\
\text { [min] }\end{array}$ & $\begin{array}{c}\text { Area } \\
{\left[\mathrm{mAU}^{*} \mathrm{~s}\right]}\end{array}$ & $\begin{array}{l}\text { Height } \\
{[\mathrm{mAU}]}\end{array}$ & $\begin{array}{c}\text { Area } \\
\%\end{array}$ \\
\hline & & & & & \\
\hline & $9.990 \mathrm{BB}$ & 6765 & 5286.34863 & 14.95294 & 97.3602 \\
\hline
\end{tabular}

\section{HPLC Chromatogram of Compound 3q}


AS-7-Br-MALONO-MIX CHIRALPAK IA-3; 250 MM

IPA/HEXANE : $15 / 85$

254 NM

FLOW RATE: $1.0 \mathrm{~mL} / \mathrm{min}$

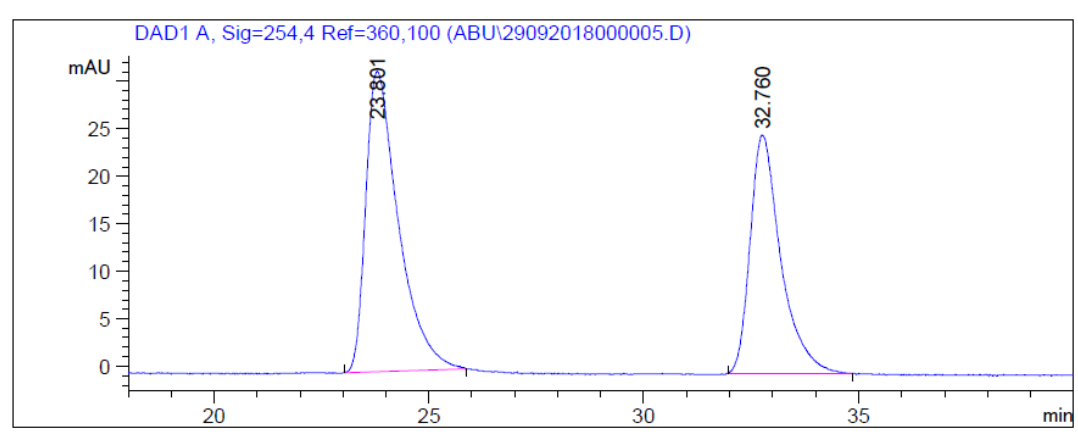

Area Percent Report

\begin{tabular}{|c|c|c|c|c|}
\hline Sorted By & : & Signal & & \\
\hline Multiplier & : & 1.0000 & & \\
\hline Dilution & : & 1.0000 & & \\
\hline \multicolumn{5}{|c|}{ Use Multiplier \& Dilution Factor with ISTDs } \\
\hline \multicolumn{5}{|c|}{ Signal 1: DAD1 A, Sig $=254,4$ Ref $=360,100$} \\
\hline $\begin{array}{l}\text { Peak RetTime Type } \\
\#[\min ]\end{array}$ & $\begin{array}{l}\text { Width } \\
\text { [min] }\end{array}$ & $\begin{array}{c}\text { Area } \\
{[\mathrm{mAU} * \mathrm{~s}]}\end{array}$ & $\begin{array}{l}\text { Height } \\
{[\mathrm{mAU}]}\end{array}$ & $\begin{array}{c}\text { Area } \\
\%\end{array}$ \\
\hline-1 & & & & \\
\hline $123.801 \mathrm{BB}$ & 0.7276 & 1673.90295 & 31.62008 & 57.3624 \\
\hline $232.760 \mathrm{BB}$ & 0.6714 & 1244.21619 & 25.05307 & 42.6376 \\
\hline
\end{tabular}

HPLC Chromatogram of Compound ( \pm ) 3r (Manual mixing)
Sample Info : AS-7-Br-MALONO-R

CHIRALPAK IA-3; 250 MM

IPA/HEXANE : $15 / 85$

254 NM

FLOW RATE: $1.0 \mathrm{~mL} / \mathrm{min}$

Additional Info : Peak(s) manually integrated

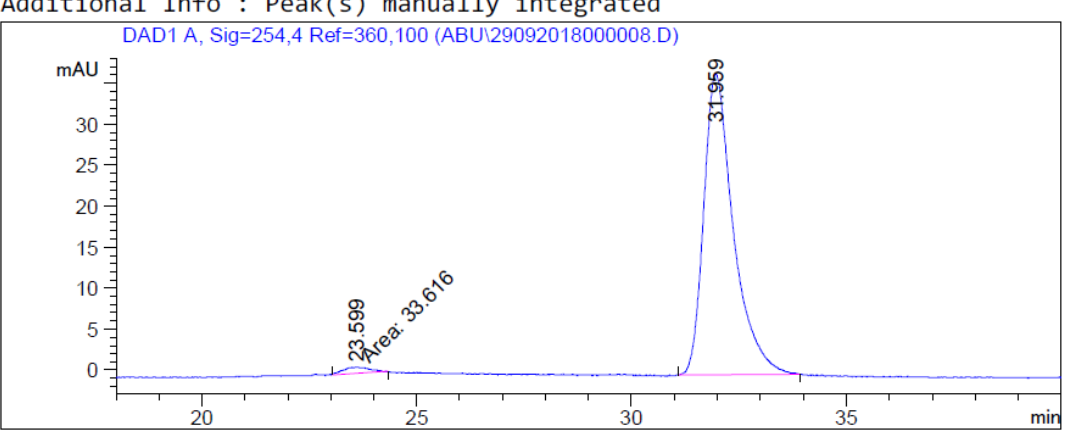

Area Percent Report

$\begin{array}{lll}\text { Sorted By } & : & \text { Signal } \\ \text { Multiplier } & : & 1.0000\end{array}$

Multiplier : : 1.0000

Dilution : 1.0000

Use Multiplier \& Dilution Factor with ISTDS

Signal 1: DAD1 A, Sig $=254,4$ Ref $=360,100$

\begin{tabular}{|c|c|c|c|c|c|}
\hline $\begin{array}{c}\text { eak } \\
\#\end{array}$ & $\begin{array}{l}\text { RetTime Type } \\
\text { [min] }\end{array}$ & $\begin{array}{l}\text { Width } \\
\text { [min] }\end{array}$ & $\begin{array}{c}\text { Area } \\
{\left[\mathrm{mAU}^{*} \mathrm{~s}\right]}\end{array}$ & $\begin{array}{l}\text { Height } \\
{[\mathrm{mAU}]}\end{array}$ & $\begin{array}{c}\text { Area } \\
\%\end{array}$ \\
\hline 1 & 99 MM & 0 & 33.61598 & $7.82018 \mathrm{e}-1$ & 1.8691 \\
\hline & $31.959 \mathrm{BB}$ & 0.6944 & 1764.94165 & 36.74612 & 98.1309 \\
\hline
\end{tabular}

HPLC Chromatogram of Compound 3r 
AS-4, 7-Di-Cl--MALONO-MIX

CHIRALPAK IA- $3 ; 250$ MM

IPA/HEXANE : $10 / 90$

254 NM

FLOW RATE: $1.0 \mathrm{~mL} / \mathrm{min}$

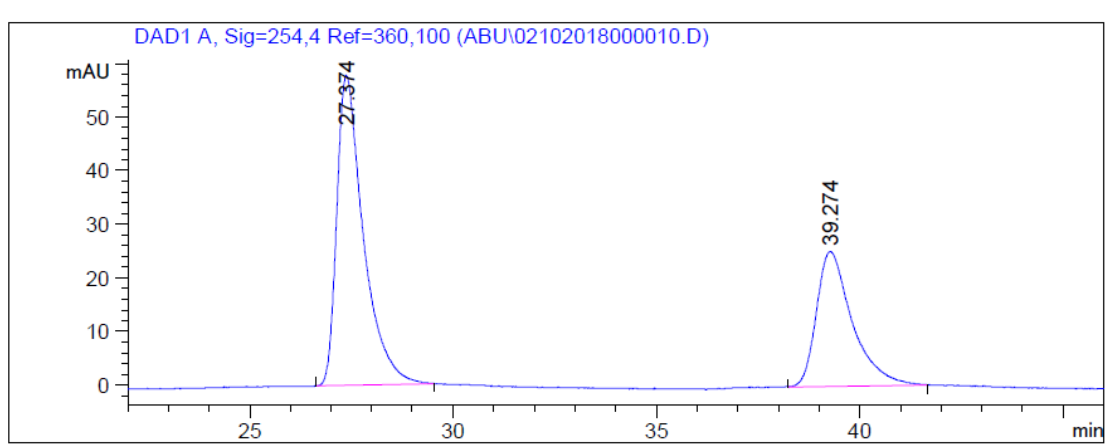

Area Percent Report

\begin{tabular}{|c|c|c|c|c|}
\hline Sorted By & : & Signal & & \\
\hline Multiplier & : & 1.0000 & & \\
\hline Dilution & : & 1.0000 & & \\
\hline \multicolumn{5}{|c|}{ Use Multiplier \& Dilution Factor with ISTDs } \\
\hline \multicolumn{5}{|c|}{ Signal 1: DAD1 A, Sig $=254,4$ Ref $=360,100$} \\
\hline $\begin{array}{l}\text { Peak RetTime Type } \\
\# \quad[\text { min] }\end{array}$ & $\begin{array}{l}\text { Width } \\
{[\text { min }]}\end{array}$ & $\begin{array}{c}\text { Area } \\
{\left[\mathrm{mAU}^{*} \mathrm{~s}\right]}\end{array}$ & $\begin{array}{l}\text { Height } \\
{[\mathrm{mAU}]}\end{array}$ & $\begin{array}{c}\text { Area } \\
\%\end{array}$ \\
\hline T & & & & \\
\hline $\begin{array}{ll}1 & 27.374 \mathrm{BB} \\
2 & 39.274 \mathrm{BB}\end{array}$ & 0.6590 & 2630.21851 & 57.79108 & 63.1834 \\
\hline $239.274 \mathrm{BB}$ & 0.8376 & 1532.61084 & 25.12584 & 36.8166 \\
\hline
\end{tabular}

HPLC Chromatogram of Compound ( \pm ) 3s (Manual mixing)
Sample Info : AS-4,7-Di-Cl--MALONO-R CHIRALPAK IA-3; 250 MM
IPA/HEXANE : $10 / 90$
254 NM

FLOW RATE: $1.0 \mathrm{~mL} / \mathrm{min}$

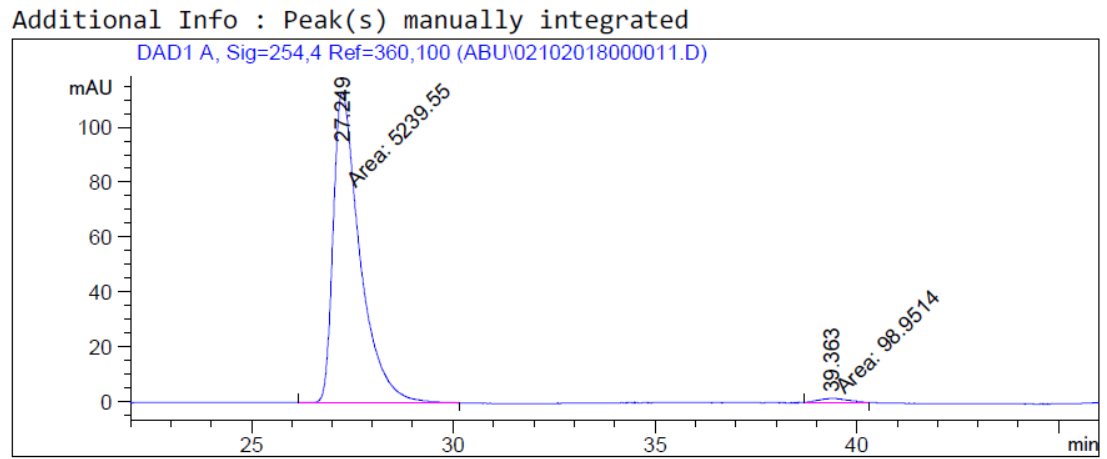

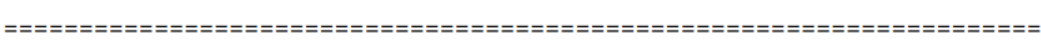
Area Percent Report

$\begin{array}{lll}\text { Sorted By } & : & \text { Signal } \\ \text { Multiplier } & : & 1.0000 \\ \text { Dilution } & : & 1.0000\end{array}$

$\begin{array}{lcc}\text { Dilution } & : & 1.0000 \\ \text { Use Multiplier \& Dilution Factor with ISTDS }\end{array}$

Signal 1: DAD1 A, Sig=254,4 Ref $=360,100$

\begin{tabular}{|c|c|c|c|c|c|}
\hline $\begin{array}{c}\text { Peak } \\
\text { \# }\end{array}$ & $\begin{array}{l}\text { RetTime Type } \\
\text { [min] }\end{array}$ & $\begin{array}{l}\text { Width } \\
\text { [min] }\end{array}$ & $\begin{array}{c}\text { Area } \\
{[\mathrm{mAU} * \mathrm{~s}]}\end{array}$ & $\begin{array}{l}\text { Height } \\
{[\mathrm{mAU}]}\end{array}$ & $\begin{array}{c}\text { Area } \\
\%\end{array}$ \\
\hline & $27249 \mathrm{MM}$ & A 7790 & & 10388 & -- \\
\hline 2 & $\begin{array}{l}27.249 \mathrm{MM} \\
39.363 \mathrm{MM}\end{array}$ & $\begin{array}{l}0.7700 \\
0.9627\end{array}$ & $\begin{array}{r}5239.55371 \\
98.95139\end{array}$ & $\begin{array}{r}113.40388 \\
1.71309\end{array}$ & $\begin{array}{r}98.1465 \\
1.8535\end{array}$ \\
\hline
\end{tabular}

HPLC Chromatogram of Compound 3s 
Sample Info

AS-Ph-N-NS-MALONO-RT-MIX

CHIRALPAK OD-H; 250 MM

IPA/HEXANE : 10/90

FLOW RATE: $1.0 \mathrm{~mL} / \mathrm{min}$

$254 \mathrm{~nm}$

Additional Info : Peak(s) manually integrated

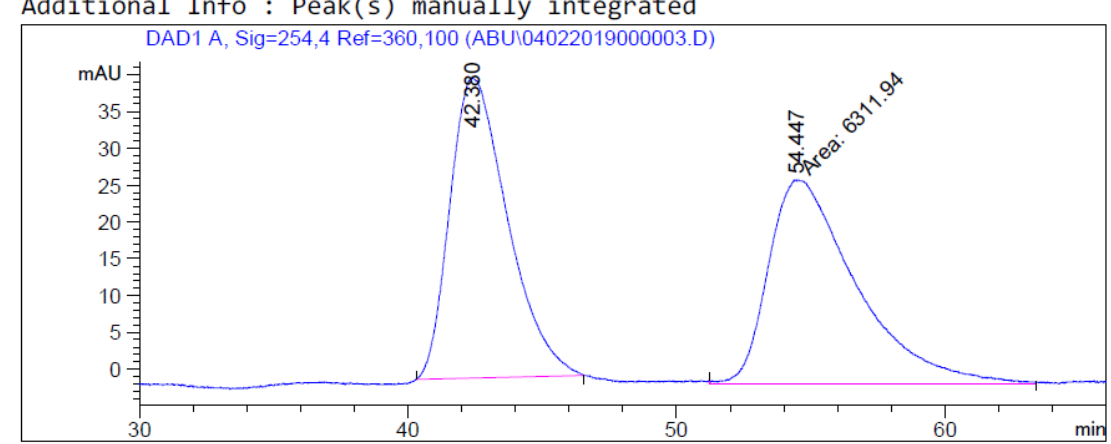

Area Percent Report

$\begin{array}{lll} & & \\ \text { Sorted By } & : & \text { Signal } \\ \text { Multiplier } & : & 1.0000 \\ \text { Dilution } & : & 1.0000\end{array}$

Use Multiplier \& Dilution Factor with ISTDs

Signal 1: DAD1 A, Sig=254,4 $\operatorname{Ref}=360,100$

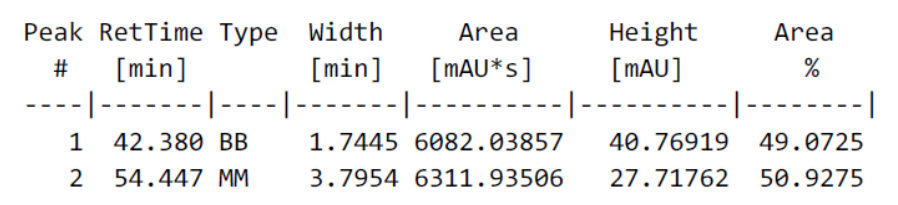

HPLC Chromatogram of Compound ( \pm ) 6a (Manual mixing)
Sample Info

AS-Ph-N-NS-MALONO-RT-L CHIRALPAK OD-H; 250 MM

IPA/HEXANE : $10 / 90$

FLOW RATE: $1.0 \mathrm{~mL} / \mathrm{min}$

$$
254 \mathrm{~nm}
$$

Additional Info : Peak(s) manually integrated

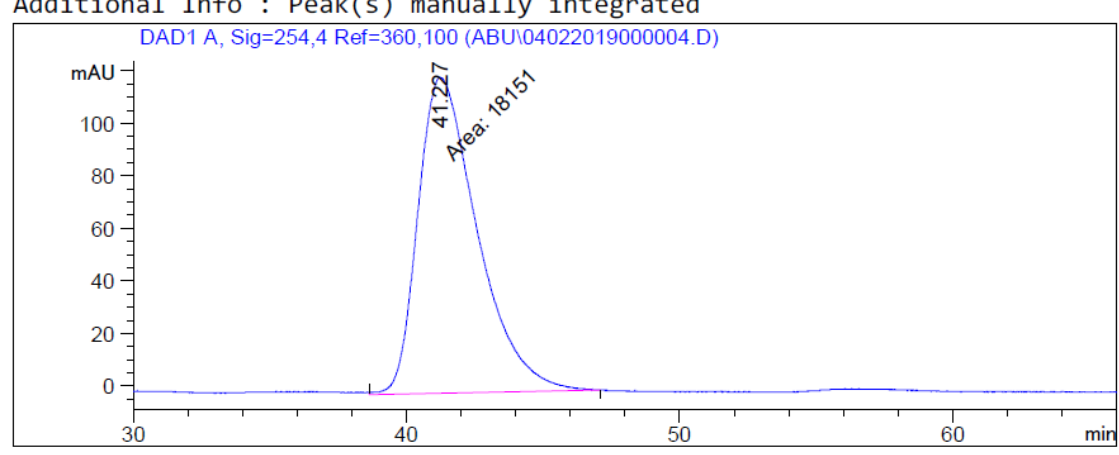

Area Percent Report

$\begin{array}{lll}\text { Sorted By } & : & \text { Signal } \\ \text { Multiplier } & : & 1.0000\end{array}$

Dilution : 1.0000

Use Multiplier \& Dilution Factor with ISTDs

Signal 1: DAD1 A, Sig=254,4 Ref=360,100

\begin{tabular}{|c|c|c|c|c|c|}
\hline $\begin{array}{c}\text { Peak } \\
\quad \#\end{array}$ & $\begin{array}{l}\text { RetTime Type } \\
\text { [min] }\end{array}$ & $\begin{array}{l}\text { Width } \\
\text { [min] }\end{array}$ & $\begin{array}{c}\text { Area } \\
{\left[\mathrm{mAU}^{*} \mathrm{~s}\right]}\end{array}$ & $\begin{array}{l}\text { Height } \\
{[\mathrm{mAU}]}\end{array}$ & $\begin{array}{c}\text { Area } \\
\%\end{array}$ \\
\hline
\end{tabular}

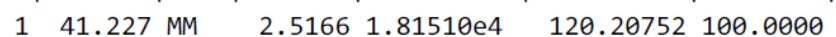

HPLC Chromatogram of Compound $\mathbf{6 a}$ 
Sample Info

: Phenyl N-Ts-MALONO-mix

CHIRALPAK IA-3; 250 MM

IPA/HEXANE : $10 / 90$

FLOW RATE: $1.0 \mathrm{~mL} / \mathrm{min}$

$254 \mathrm{~nm}$

Additional Info : Peak(s) manually integrated

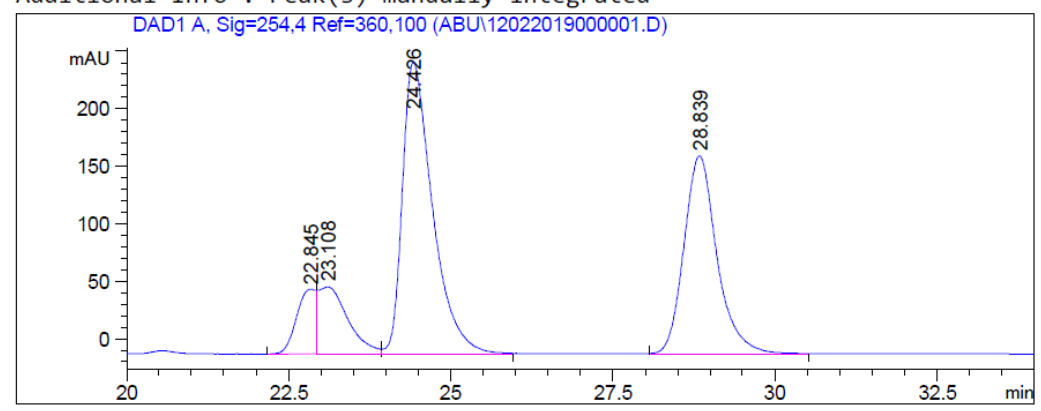

Area Percent Report

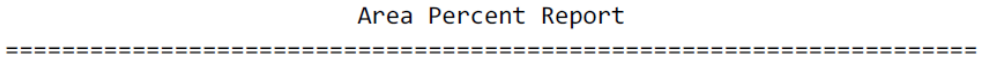

$\begin{array}{lll}\text { Sorted By } & : & \text { Signal } \\ \text { Multiplier } & : & 1.0000 \\ \text { Dilution } & : & 1.0000\end{array}$

Dilution : 1.0000

Use Multiplier \& Dilution Factor with ISTDs

Signal 1: DAD1 A, Sig $=254,4$ Ref $=360,100$

\begin{tabular}{|c|c|c|c|c|c|c|}
\hline eak & $\begin{array}{c}\text { RetTime } \\
\text { [min] }\end{array}$ & & $\begin{array}{l}\text { Width } \\
\text { [min] }\end{array}$ & $\begin{array}{c}\text { Area } \\
{[\mathrm{mAU} * \mathrm{~s}]}\end{array}$ & $\begin{array}{l}\text { Height } \\
{[\mathrm{mAU}]}\end{array}$ & $\begin{array}{c}\text { Area } \\
\%\end{array}$ \\
\hline & & & & & & 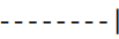 \\
\hline 1 & 22.8 & $\mathrm{BV}$ & .31 & 1135.59 & 56.11348 & 6.5924 \\
\hline 2 & 3. & $\mathrm{VV}$ & 46 & 47.6 & 58.2 & 10.7262 \\
\hline 3 & 24.426 & D & 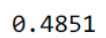 & 80.47 & 253.08585 & 47.4891 \\
\hline & & & & & & \\
\hline
\end{tabular}

HPLC Chromatogram of Compound ( \pm ) $\mathbf{6 b}$ (Manual mixing)
Sample Info : Phenyl N-Ts-MALONO-L CHIRALPAK IA-3; 250 MM IPA/HEXANE : $10 / 90$ FLOW RATE: $1.0 \mathrm{~mL} / \mathrm{min}$ $254 \mathrm{~nm}$

Additional Info : Peak(s) manually integrated

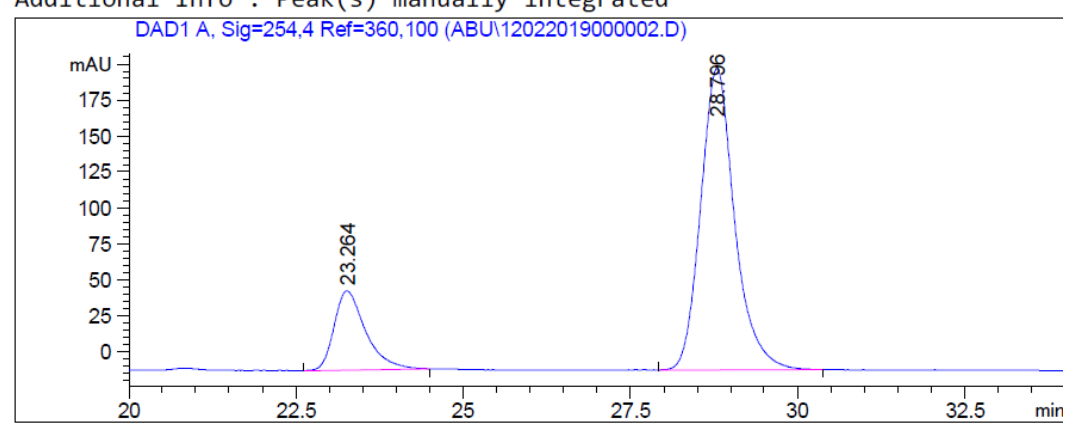

22.5

Area Percent Report

$\begin{array}{lll}\text { Sorted By } & : & \text { Signal } \\ \text { Multiplier } & : & 1.0000 \\ \text { Dilution } & : & 1.0000\end{array}$

Use Multiplier \& Dilution Factor with ISTDs

Signal 1: DAD1 A, Sig=254,4 Ref $=360,100$

\begin{tabular}{|c|c|c|c|c|c|}
\hline $\begin{array}{l}\text { eak } \\
\#\end{array}$ & $\begin{array}{l}\text { RetTime Type } \\
\text { [min] }\end{array}$ & $\begin{array}{l}\text { Width } \\
\text { [min] }\end{array}$ & $\begin{array}{c}\text { Area } \\
{\left[\mathrm{mAU}^{*} \mathrm{~s}\right]}\end{array}$ & $\begin{array}{l}\text { Height } \\
{[\mathrm{mAU}]}\end{array}$ & $\begin{array}{c}\text { Area } \\
\%\end{array}$ \\
\hline & & & & & \\
\hline & & & & 10.01038 & \\
\hline
\end{tabular}

HPLC Chromatogram of Compound $\mathbf{6 b}$ 


$$
\begin{array}{ll}
\text { Sample Info } & \text { RAC-N-Me-malono } \\
& \text { CHIRALPAK IA-3; 250 MM } \\
& \text { IPA/HEXANE }: 20 / 80 \\
& \text { FLOW RATE: } 1.0 \mathrm{~mL} / \mathrm{min} \\
& 254 \mathrm{~nm}
\end{array}
$$

Additional Info : Peak(s) manually integrated

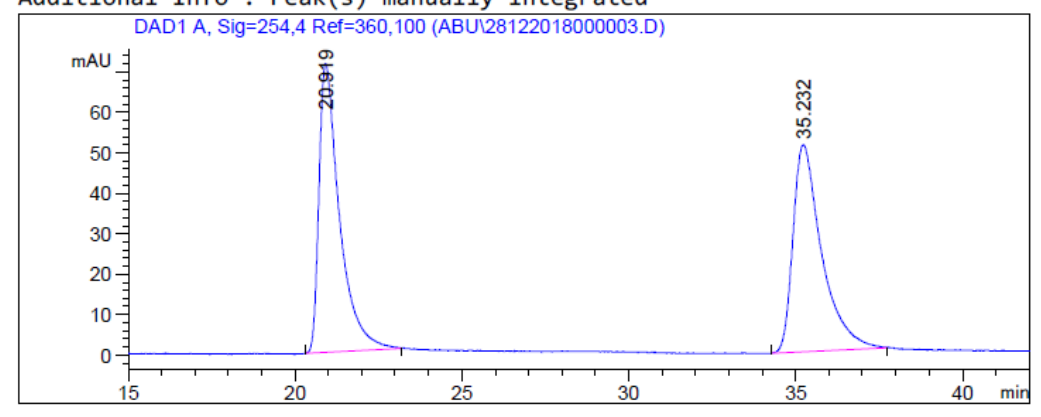

Area Percent Report

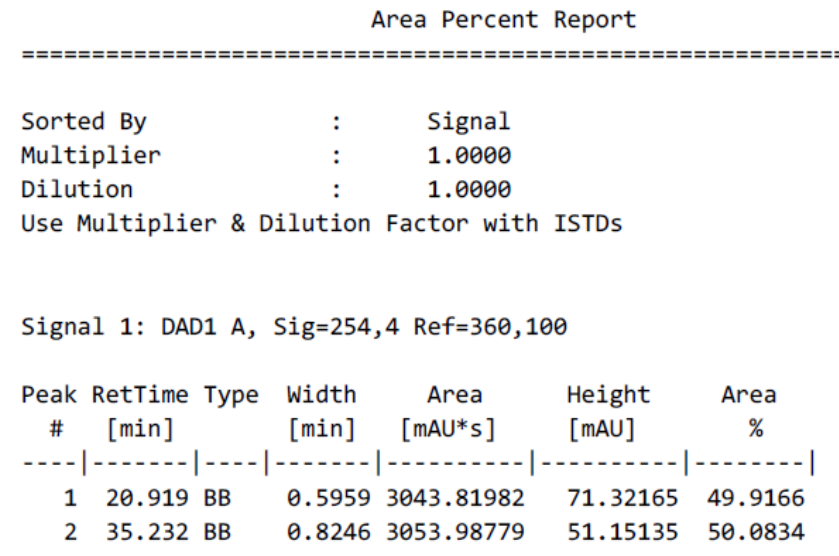

HPLC Chromatogram of Compound ( \pm ) 3a
RAC-N-Me-malono-dcm-quinine- $\theta$ degree

CHIRALPAK IA-3; 250 MM

IPA/HEXANE : 20/80

FLOW RATE: $1.0 \mathrm{~mL} / \mathrm{min}$

$254 \mathrm{~nm}$

Additional Info : Peak(s) manually integrated

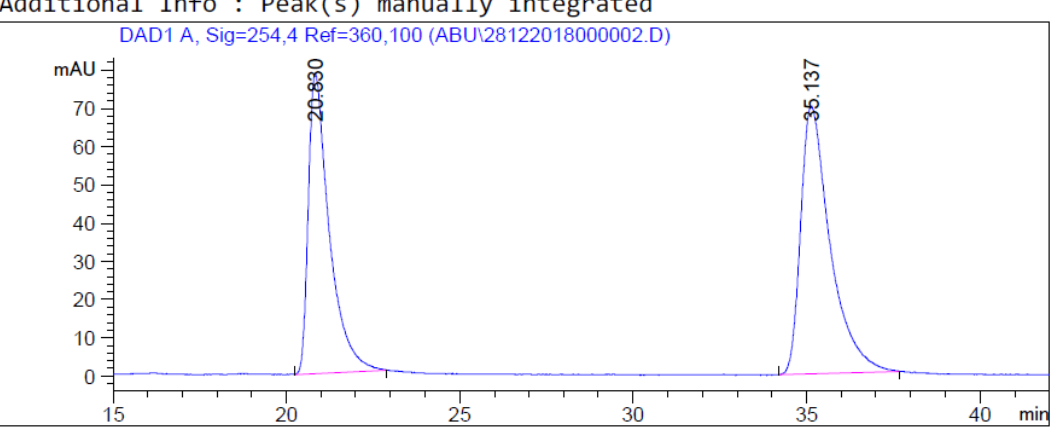

Area Percent Report

\begin{tabular}{|c|c|c|c|c|c|}
\hline $\begin{array}{l}\text { ak } \\
\text { a }\end{array}$ & $\begin{array}{l}\text { RetTime Type } \\
\text { [min] }\end{array}$ & $\begin{array}{l}\text { Width } \\
\text { [min] }\end{array}$ & $\begin{array}{c}\text { Area } \\
{[\mathrm{mAU} * \mathrm{~s}]}\end{array}$ & $\begin{array}{l}\text { Height } \\
{[\mathrm{mAU}]}\end{array}$ & $\begin{array}{c}\text { Area } \\
\%\end{array}$ \\
\hline & & & & & \\
\hline & $\mathrm{BB}$ & 831 & .09570 & 70.07424 & 5.7506 \\
\hline
\end{tabular}

$\begin{array}{lll}\text { Sorted By } & : & \text { Signal } \\ \text { Multiplier } & : & 1.0000 \\ \text { Dilution } & : & 1.0000\end{array}$

Dilution : 1.0000

Use Multiplier \& Dilution Factor with ISTDs

Signal 1: DAD1 A, Sig $=254,4$ Ref $=360,100$

HPLC Chromatogram of Compound 3a 
CHIRALPAK IB-3; 250 MM

IPA/HEXANE : 10/90

FLOW RATE: $1.0 \mathrm{~mL} / \mathrm{min}$ $254 \mathrm{~nm}$

Additional Info : Peak(s) manually integrated

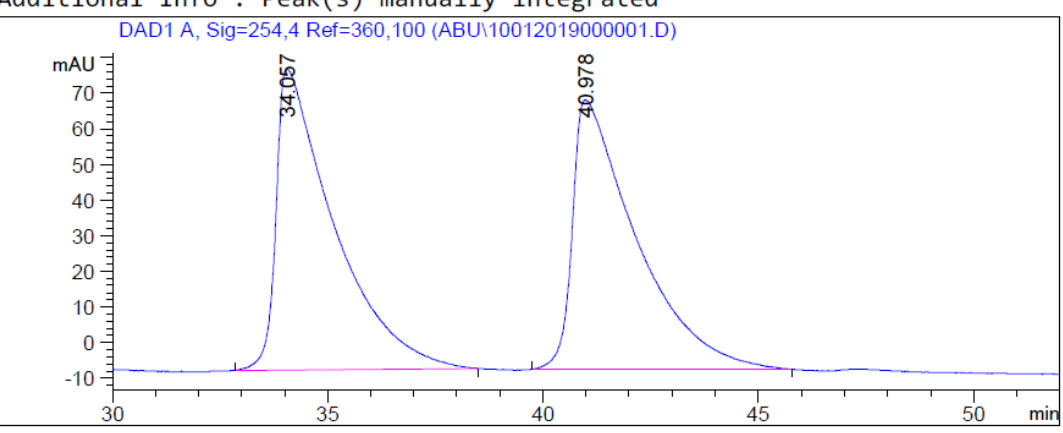

Additional Info : Peak(s) manually integrated

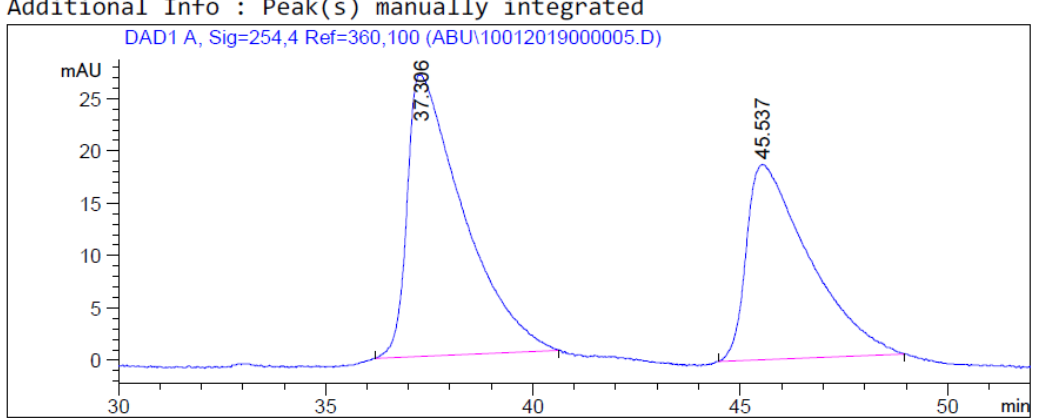

Area Percent Report

Area Percent Report

$\begin{array}{lll}\text { Sorted By } & : & \text { Signal } \\ \text { Multiplier } & : & 1.0000 \\ \text { Dilution } & : & 1.0000\end{array}$

Use Multiplier \& Dilution Factor with ISTDS

Signal 1: DAD1 A, Sig=254,4 Ref=360,100

\begin{tabular}{|c|c|c|c|c|c|}
\hline $\begin{array}{c}\text { Peak } \\
\#\end{array}$ & $\begin{array}{l}\text { RetTime Type } \\
\text { [min] }\end{array}$ & $\begin{array}{l}\text { Width } \\
\text { [min] }\end{array}$ & $\begin{array}{c}\text { Area } \\
{[\mathrm{mAU} * \mathrm{~s}]}\end{array}$ & $\begin{array}{l}\text { Height } \\
{[\mathrm{mAU}]}\end{array}$ & $\begin{array}{c}\text { Area } \\
\%\end{array}$ \\
\hline & & & & & \\
\hline 2 & 40.978 BB & 80 & 7782.78223 & 75.66447 & 49.6946 \\
\hline
\end{tabular}

\section{HPLC Chromatogram of Compound ( \pm ) 1a}


Additional Info : Peak(s) manually integrated

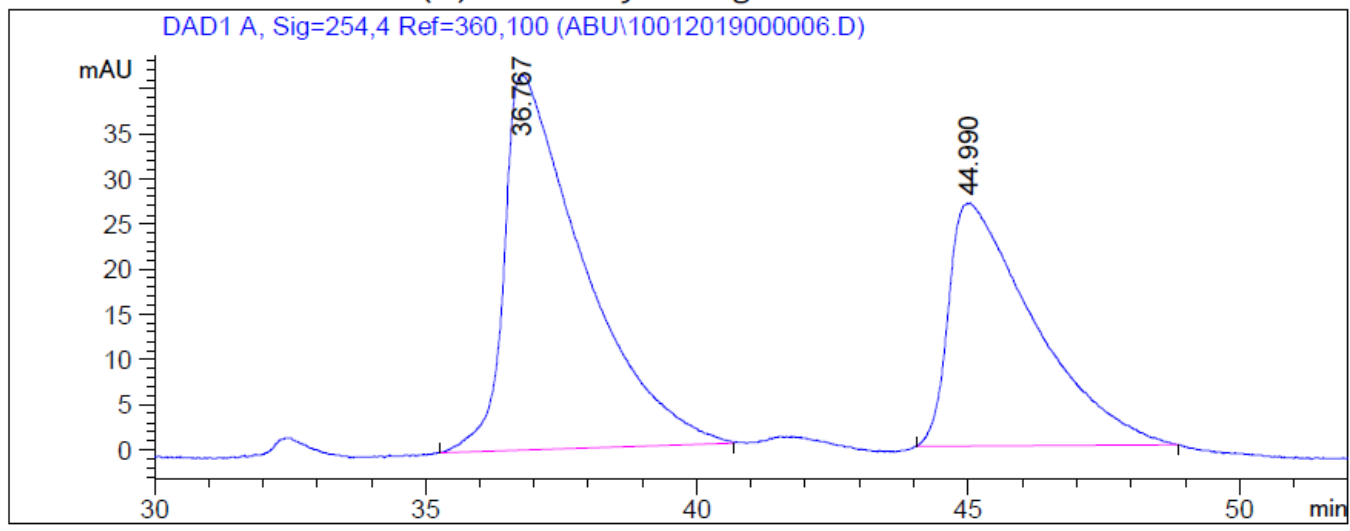

Area Percent Report

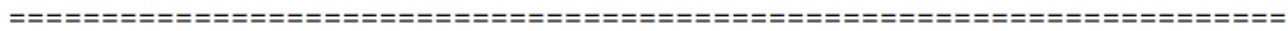

$\begin{array}{lll}\text { Sorted By } & : & \text { Signal } \\ \text { Multiplier } & : & 1.0000 \\ \text { Dilution } & : & 1.0000\end{array}$

Use Multiplier \& Dilution Factor with ISTDS

Signal 1: DAD1 A, Sig=254,4 Ref $=360,100$

\begin{tabular}{|c|c|c|c|c|c|c|}
\hline $\begin{array}{l}\text { eak } \\
\#\end{array}$ & $\begin{array}{c}\text { RetTime } \\
\text { [min] }\end{array}$ & Type & $\begin{array}{l}\text { Width } \\
\text { [min] }\end{array}$ & $\begin{array}{c}\text { Area } \\
{\left[\mathrm{mAU}^{*} \mathrm{~s}\right]}\end{array}$ & $\begin{array}{l}\text { Height } \\
{[\mathrm{mAU}]}\end{array}$ & $\begin{array}{c}\text { Area } \\
\%\end{array}$ \\
\hline & & & & & & \\
\hline 1 & & & & 422 & 32 & 20 \\
\hline 2 & 44.990 & 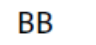 & 903 & 2961.29810 & 26.89978 & 41 \\
\hline
\end{tabular}

HPLC Chromatogram of Compound recovered 1a (18\% ee) 